\title{
Novel methods for the detection of functional brain activity using $170 \mathrm{MRI}$
}

Citation for published version (APA):

Möllenhoff, K. (2016). Novel methods for the detection of functional brain activity using $170 \mathrm{MRI}$. [Doctoral Thesis, Maastricht University, Université de Liège]. Maastricht University.

https://doi.org/10.26481/dis.20160620km

Document status and date:

Published: 01/01/2016

DOI:

10.26481/dis.20160620km

Document Version:

Publisher's PDF, also known as Version of record

\section{Please check the document version of this publication:}

- A submitted manuscript is the version of the article upon submission and before peer-review. There can be important differences between the submitted version and the official published version of record.

People interested in the research are advised to contact the author for the final version of the publication, or visit the DOI to the publisher's website.

- The final author version and the galley proof are versions of the publication after peer review.

- The final published version features the final layout of the paper including the volume, issue and page numbers.

Link to publication

\footnotetext{
General rights rights.

- You may freely distribute the URL identifying the publication in the public portal. please follow below link for the End User Agreement:

www.umlib.nl/taverne-license

Take down policy

If you believe that this document breaches copyright please contact us at:

repository@maastrichtuniversity.nl

providing details and we will investigate your claim.
}

Copyright and moral rights for the publications made accessible in the public portal are retained by the authors and/or other copyright owners and it is a condition of accessing publications that users recognise and abide by the legal requirements associated with these

- Users may download and print one copy of any publication from the public portal for the purpose of private study or research.

- You may not further distribute the material or use it for any profit-making activity or commercial gain

If the publication is distributed under the terms of Article $25 \mathrm{fa}$ of the Dutch Copyright Act, indicated by the "Taverne" license above, 


\title{
J) JÜLICH Maastricht University

\section{NOVEL METHODS FOR THE DETECTION OF FUNCTIONAL BRAIN ACTIVITY USING ${ }^{17} \mathrm{O} \mathrm{MRI}$}

\author{
DISSERTATION \\ to obtain the joint degree of Doctor at \\ Université de Liège and Maastricht University \\ on the authority of the Rector Magnifici, \\ Prof. A. Corhay and Prof. Dr. L.L.G Soete \\ in accordance with the decision of the Board of Deans, \\ to be defended in public in Maastricht \\ on Monday the $20^{\text {th }}$ of June 2016 at $16: 00$ hours \\ by \\ Klaus Hans Manfred Möllenhoff
}




\section{Supervisors}

Prof. Dr. Nadim Jon Shah

Forschungszentrum Jülich - Germany \& Maastricht University - Netherlands

Ir. Dr. Christophe Phillips

Université de Liège - Belgium

Dr. Evelyne Balteau

Université de Liège - Belgium

\section{Assessment Committee}

Prof. Dr. Michael Kraft, Université de Liège (Chairman)

Prof. Dr. Walter Backes, Maastricht University

Prof. Dr. Ralf Deichmann, Goethe University of Frankfurt

Prof. Dr. Armin Nagel, University of Erlangen \& DKFZ Heidelberg

(c) Klaus Hans Manfred Möllenhoff, 2016

The work presented in this thesis was funded by the EU within the PEOPLE Programme (FP7): Initial Training Networks (FP7-PEOPLE-ITN-2008), Grant Agreement No. 238593 NEUROPHYSICS, in which Maastricht University, Université de Liège, Forschungszentrum Jülich $\mathrm{GmbH}$ and GlaxoSmithKline Ltd were network partners. The Ph.D. research was conducted primarily at the Forschungszentrum Jülich $\mathrm{GmbH}$. 
To my father 


\section{Contents}

1 Introduction 3

2 Theoretical Background $\quad 7$

2.1 Hardware - Components of an MR Scanner . . . . . . . . . . . . . . 7

2.1.1 Superconducting Magnet . . . . . . . . . . . . . . . . . 7

2.1.2 Radiofrequency and Magnetic Shielding . . . . . . . . . . 8

2.1.3 Shimming and Shim Coils . . . . . . . . . . . . . . . . . . . 8

2.1 .4 Gradient Coils . . . . . . . . . . . . . . . . . . . . . . . . . . 9

2.1 .5 RF Coils . . . . . . . . . . . . . . . . . . . . . . . . . . 9

2.2 NMR Phenomenon . . . . . . . . . . . . . . . . . . . . . . . 11

2.2.1 Spin, Magnetisation and Larmor Precession . . . . . . . . . . . . 11

2.2 .1 .1 Spin . . . . . . . . . . . . . . . . . 12

2.2.1.2 Interactions with a Static Magnetic Field . . . . . . . . 13

2.2.1.2.1 Magnetisation . . . . . . . . . . . . . . . 14

2.2.1.2.2 Precession . . . . . . . . . . . . . . . . 15

2.2.2 Resonance: RF Excitation and Free Induction Decay . . . . . . . . . 16

2.2.2.1 Interaction with a Radiofrequency Field . . . . . . . . 17

2.2.3 Spin Density, Relaxation, Saturation and MR Contrast . . . . . . . . 19

2.2.3.1 Mathematical description of relaxation processes . . . . . 21

2.2.3.1.1 Longitudinal Spin-Lattice Relaxation . . . . . . . 21

2.2.3.1.2 Transverse Spin-Spin Relaxation ... . . . . . 22

2.2.3.1.3 Bloch Equation . . . . . . . . . . . . . 22

2.3 MR Imaging and MR Sequences . . . . . . . . . . . . . . . . . 23

2.3.1 Principles of Magnetic Resonance Imaging . . . . . . . . . . . . . 23

2.3.1.1 k-space and FT - 2D and 3D . . . . . . . . . 23

2.3.1.1.1 Fourier Transform . . . . . . . . . . . . . . 23

2.3.1.2 Selective Excitation and Spatial Encoding . . . . . . . . 24

2.3.1.2.1 Spatial Resolution and Field-of-View . . . . . . . 26

2.3.1.2.2 RF Excitation . . . . . . . . . . . . . . . . . 28

2.3.1.2.3 Free Induction Decay . . . . . . . . . . . . 31

2.3.1.2.4 Gradient encoding . . . . . . . . . . . . . . . 32

2.3.2 Influence of Sequence Parameters on Image Quality . . . . . . . . 36

2.3.3 Echoes: Spin Echoes and Gradient Echoes . . . . . . . . . . . . . 37

2.3.3.1 Echoes . . . . . . . . . . . . . . . . . . . 38

2.3.4 Data acquisition in 2D and 3D . . . . . . . . . . . . . . 39

2.3.4.1 2-dimensional . . . . . . . . . . . . . . . . . . . . . 39

2.3.4.2 3-dimensional . . . . . . . . . . . . . 40 
2.3.4.3 A simple imaging sequence . . . . . . . . . . . . . . . . . 40

2.3.5 Artefacts . . . . . . . . . . . . . . . . . 41

2.3.5.1 Aliasing (Wrap-around) . . . . . . . . . . . . . . 41

2.3.5.2 Chemical Shift . . . . . . . . . . . . . . . . . . . . 42

2.3.5.3 $\mathrm{B}_{1}$ Inhomeogeneities ................ . . 42

2.3.6 Adiabatic pulses . . . . . . . . . . . . . . . . . . 43

2.3.6.1 Basics ... . . . . . . . . . . . . . . . 43

2.3.6.2 Inversion Pulses . . . . . . . . . . . . . . . . . . . 45

2.3.6.3 Adiabatic RF Pulses with less peak power . . . . . . . . . 46

2.3.7 Parallel Imaging Techniques . . . . . . . . . . . . . . . . . . 48

2.3.7.1 History of Parallel Imaging Reconstruction . . . . . . . . . 48

2.3.7.2 GRAPPA Reconstruction . . . . . . . . . . . . . . . . . . 49

2.3.8 Multi Echoes and EPI Factor . . . . . . . . . . . . . . . . . . 50

2.3.8.1 Echotimeshifting ............... . . 52

2.3.9 Multi-shot multi-echo spin-echo EPI . . . . . . . . . . . . . . . 54

2.4 Safety Aspects . . . . . . . . . . . . . . . . . . . . . . . . . . . . . . . 54

2.5 Oxygen Metabolism . . . . . . . . . . . . . . . . . . . . . . . . 56

2.5.1 Oxygen Transport . . . . . . . . . . . . . . . . . 56

2.5.2 Electron Transport Chain . . . . . . . . . . . . . . . . . 56

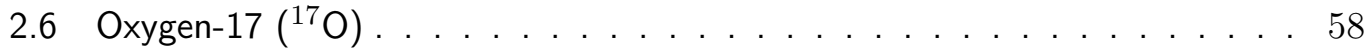

3 Methods $\quad 61$

3.1 Quantification of $\mathrm{CMRO}_{2} \ldots . . . . . . . . . . . . . . .661$

3.1.1 Cerebral metabolic rate of oxygen consumption . . . . . . . . . . 61

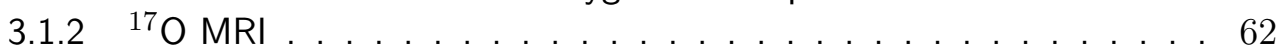

3.1.3 Quantification of $\mathrm{CMRO}_{2}$ based on ${ }^{17} \mathrm{O}-\mathrm{MRI} . . . . . . . .63$

3.1.3.1 $\mathrm{CMRO}_{2}$ quantification based on a 3-phase measurement . 64

3.1.4 ${ }^{1} \mathrm{H}-\mathrm{MRI}$. . . . . . . . . . . . . . . . . . 65

3.1.4.1 From venous blood $T_{2}$ to $Y_{v}$ values . . . . . . . . . . 66

3.1.4.2 Multi Echo Spin Echo . . . . . . . . . . . . . . . . . 67

3.1.4.2.1 Stimulated Echoes ............ . . 68

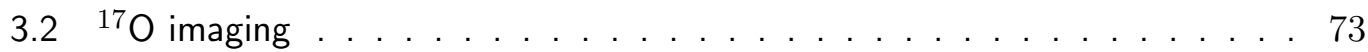

3.3 Quantitative Imaging .. . . . . . . . . . . . . . . . . 75

3.3.1 Motivation ...................... . . . . . . . . . . . . . . . . .

3.3.2 Mapping of Relaxation Times . . . . . . . . . . . . . . . . . . 76

3.3.2.1 Gold Standard - Spectroscopic Measurement of Relaxation

Parameters ... . . . . . . . . . . . . 76

3.3.2.2 TAPIR sequence . . . . . . . . . . . . . . . . . . 77

3.3.3 Water Mapping . . . . . . . . . . . . . . . . . . . 82

3.3.3.1 $M_{0}$ Maps ..................... . . 82

3.3.3.2 $T_{2}^{*}$ Correction . . . . . . . . . . . . . . . . . . . . 82

3.3.3.3 Receiver Coil Sensitivity Correction . . . . . . . . . . . . . 83

3.3.3.4 Calibration to CSF ................. . 84

3.3.3.5 Data Processing . . . . . . . . . . . . . . . . . . 86

3.4 Sequence Design . . . . . . . . . . . . . . . . . . . . . . . . . . . . . 87

3.4.1 Twisted Projection Imaging - TPI . . . . . . . . . . . . . . . 87 
3.4.2 $T_{1}$ Mapping with TAPIR . . . . . . . . . . . . . . . . 89

3.4.2.1 Inversion Efficiency and Adiabatic Inversion Pulses . . . . 89

3.4.2.2 Parallel Imaging Combined with EPI Readout . . . . . . . 90

3.4.2.3 QUIXOTIC .................... . . 91

3.4.2.3.1 Venular-Targeted Velocity-Selective Spin Labelling 91

3.4.2.3.2 Measurement Setup . . . . . . . . . . . . . . 93

4 Experiments and Results $\quad 97$

4.1 Mapping of Absolute Water Content and Longitudinal Relaxation Times . . 97

4.1 .1 Sequence Parameters . . . . . . . . . . . . . . . . . . . 97

4.1 .2 Phantom Results . . . . . . . . . . . . . . . . . . . . . . . . . . 98

4.1 .3 In Vivo Results . . . . . . . . . . . . . . . . . . . . . . . . . . . . . 98

4.1.4 Discussion and Conclusions . . . . . . . . . . . . . . . . . . . . 101

4.2 Natural Abundance ${ }^{17} \mathrm{O}$ MRI . . . . . . . . . . . . . . . . . . . . . . 103

4.2.1 Natural Abundance ${ }^{17} \mathrm{O}$ MRI in vivo . . . . . . . . . . . . . . . 103

4.2.2 Natural Abundance ${ }^{17} \mathrm{O}$ MRI in phantom . . . . . . . . . . . . . 105

4.2.3 Discussion and Conclusions . . . . . . . . . . . . . . . 107

4.3 Quantification of $\mathrm{CMRO}_{2} \ldots \ldots \ldots$. . . . . . . . . . . . . . . . . 109

4.3.1 $T_{2}$ Measurements . . . . . . . . . . . . . . . . . . . . 109

4.3 .2 QUIXOTIC . . . . . . . . . . . . . . . . . . . . . 109

4.3.3 Discussion and Conclusions . . . . . . . . . . . . . . . . . . 111

5 Discussion, Conclusions and Outlook 113

$\begin{array}{lll}6 & \text { Acknowlegdements } & 115\end{array}$

$\begin{array}{ll}\text { A Curriculum Vitae } & 117\end{array}$

$\begin{array}{lr}\text { B Valorisation } & 119\end{array}$

B.1 Social and Economic Relevance . . . . . . . . . . . . . . . . . . . 119

B.2 Target Group . . . . . . . . . . . . . . . . . . . . . . . . . 121

B.3 Products and Services . . . . . . . . . . . . . . . . . . . . . 121

B.4 Innovation . . . . . . . . . . . . . . . . . . . . . . . . . . . 122

B.5 Shedule and Implementation . . . . . . . . . . . . . . . . . . 123 


\section{Abstract}

Detailed quantitative information about metabolic processes plays a crucial role in the potential cure and for treatment of many diseases such as Alzheimer's disease or brain tumours. In the last decades, radioactive tracers such as ${ }^{15} \mathrm{O}$ have been used to quantify $\mathrm{CMRO}_{2}$ with PET imaging and this is regarded as the gold standard. However, such methods are complicated and expensive as a consequence of the short half-life $(2 \mathrm{~min})$ of ${ }^{15} \mathrm{O}$ and inherently include radiation exposure and invasive measurements such as blood probes to probe cerebral blood flow (CBF). Fick's principle of arteriovenous oxygen difference [1] connects $\mathrm{CMRO}_{2}$ and $\mathrm{CBF}$ via the measure of oxygen extraction fraction (OEF).

The main goal of this work is to achieve non-invasive measures of OEF based on magnetic resonance imaging (MRI) to quantify $\mathrm{CMRO}_{2}$ allowing straightforward and comfortable patient handling. MRI enables studies of large cohorts of healthy volunteers due to noninvasive measurements and a lack of radioactivity. This can be achieved first by quantitative relaxation time mapping of the transverse relaxation time $\left(\mathrm{T}_{2}\right)$ of venous blood only in proton $\left({ }^{1} \mathrm{H}\right) \mathrm{MRI}$ or by a measurement following inhalation of ${ }^{17} \mathrm{O}$ gas and recording the signal curve of directly detected ${ }^{17} \mathrm{O}$ signal. Unfortunately, the most abundant isotope of oxygen $\left({ }^{16} \mathrm{O}\right)$ has a zero spin system, and cannot be detected with NMR experiments. In contrast, ${ }^{17} \mathrm{O}$, a stable isotope with a half-integer spin $(I=5 / 2)$, can be detected by MR. Fortuitously, however, in $\mathrm{MRI}$ it is only visible in the form of metabolically generated $\mathrm{H}_{2}^{17} \mathrm{O}$ and not as a gas. The low natural abundance of ${ }^{17} \mathrm{O}$, of only $0.037 \%$ (of the oxygen atoms) and the low NMR sensitivity $\left(2.9 \%\right.$ that of $\left.{ }^{1} \mathrm{H}\right)$ gives rise to the need for ultra-high-field MRI to reach a significant SNR per unit time.

Natural abundance images of a healthy male volunteer were acquired in vivo after having gained written consent within a clinical trial of a 9.4 T MRI system (Siemens AG, Erlangen, Germany) [2,3]. These natural abundance images, which reflect the ${ }^{17} \mathrm{O}$ bound to protons as $\mathrm{H}_{2}^{17} \mathrm{O}$ and thus, the amount of water, are compared to ${ }^{1} \mathrm{H}$-based quantitative water content imaging. For further studies, the voxelwise knowledge of the quantitative water content is necessary to quantify $\mathrm{CMRO}_{2}$ based on the ${ }^{17} \mathrm{O}$ signal behaviour. To achieve that, methods which were originally used on $1.5 \mathrm{~T}$ scanners had to be adapted for the use at higher field strengths to overcome RF field inhomogeneities [4-11]. New correction methods were developed based on a well known correlation between tissue $T_{1}$ and proton density (PD) to estimate the receive bias field properly. These methods were tested for quantitative water content determination. Averaged results in grey (GM) and white matter (WM) respectively of 10 healthy volunteers are $\mathrm{H}_{2} \mathrm{O}_{W M}=70.3 \pm 1.4 \%$, $\mathrm{H}_{2} \mathrm{O}_{\mathrm{GM}}=84.7 \pm 1.5 \%, T_{1_{\mathrm{WM}}}=918 \pm 24 \mathrm{~ms}$ and $T_{1_{\mathrm{GM}}}=1509 \pm 14 \mathrm{~ms}$.

Further, ${ }^{1} \mathrm{H}$-based imaging methods called QUIXOTIC [12-14] and TRUST [15] appeared in the literature. These methods are based on changes of the proton transverse relaxation rate $T_{2}$ with different oxygen saturation levels. Quantitative values of venous blood $T_{2}$ were acquired using a so-called T2prep module or a multi-echo spin echo readout. While the first method suffers from long acquisition times the latter one from large echo-spacing of the spin echoes and stimulated echo effects. Both disadvantages were overcome using an adiabatic multi-shot multi-echo spin echo sequence, which does not suffer from stimulated echo effects and due to the multi-shot capabilities, the echo-spacing is reduced [16]. Mean values in GM of four healthy volunteers are found to be venous oxygenation $\mathrm{Y}_{v}=0.61 \pm 0.03$, $\mathrm{T}_{2}=54 \pm 4 \mathrm{~ms}, \mathrm{CMRO}_{2}=174 \pm 13 \mu \mathrm{mol} / 100 \mathrm{~g} \mathrm{~min}$ and $\mathrm{CBF}=53 \pm 3 \mathrm{ml} / 100 \mathrm{~g} \mathrm{~min}$. 


\section{Chapter 1}

\section{Introduction}

Metabolic processes and their fault-free function play a crucial role in the human body. In many diseases such as Alzheimer's disease, multiple sclerosis or in brain tumours, metabolic processes - and especially the metabolisation of oxygen - are disturbed. In this regard, quantitative knowledge of the cerebral metabolic rate of oxygen consumption $\left(\mathrm{CMRO}_{2}\right)$ is of great interest for diagnostics and therapy.

According to Fick's principle [1], it is possible to calculate the blood flow to an organ using a marker substance if the amount of the marker substance and its concentration in arterial blood and in venous blood is known. In the original method, the "organ" was the entire human body and the marker substance was oxygen. This can be applied to the human brain and thus the arteriovenous oxygen difference connects $\mathrm{CMRO}_{2}$ and $\mathrm{CBF}$ via the parameter oxygen extraction fraction (OEF).

Since decades, positron emission tomography (PET), a radioactive and invasive medical imaging method, is the method of choice for obtaining quantitative values of e.g. $\mathrm{CMRO}_{2}$ and cerebral blood flow (CBF). Recently, an MRI-based method called arterial spin labelling (ASL), has been developed and used to quantify CBF quantitatively by validation through simultaneously acquired PET data [17-19].

Nuclear magnetic resonance (NMR), the basic physical principles of which are described in section 2.2, has been widely used in chemistry and physics for several decades for studying, for example, molecular structures. It was discovered independently by Felix Bloch [20] and Edward Purcell [21] for which they were awarded the Nobel Prize in Physics in 1952. During the 1970s, magnetic resonance imaging (MRI) was developed by Lauterbur [22] and Mansfield [23] for which they were also awarded the Nobel Prize in Medicine in 2003. In the interim, MRI has grown into one of the most important non-invasive imaging techniques in medicine. This is not only because of the non-invasiveness of this method, but also because of the richness of the attainable tissue contrast. This, in turn, is due to a number of contrast mechanisms which reflect, albeit in a very convoluted way, the microscopic properties of tissue. The observation by Damadian in 1971 [24] that tumours have different 
NMR relaxation properties from healthy tissue, provided much of the impetus behind the development of MRI as a diagnostic tool. MRI is now a ubiquitous imaging modality that provides rich and versatile tissue contrast and its success is deeply rooted in the fact that image contrast can be changed as desired, under electronic control, while the method itself is non-invasive.

As previously mentioned, the huge advantage of MRI in comparison to other imaging modalities lies in its potential for generating versatile contrasts between different tissue types through a careful choice of measurement parameters. This so-called "weighting" of the images results in, for example, the so-called $T_{1}$-weighted (longitudinal relaxation time $T_{1}$ ) images. For example, spin density or relaxation time weighted images are extensively used in clinical applications. These high-resolution images are useful in the clinics if pathology resulting in anatomical change is present. In contrast to radioactive tomography methods such as PET, MRI is not normally used in a quantitative manner. With the knowledge of MR imaging principles (described in Chapter 2), which is a prerequisite for using MRI in interdisciplinary fields such as molecular imaging, quantitative values of different tissue properties or even of metabolic processes can be estimated.

The main goal of this work is to perform non-invasive measurement of OEF based on MRI to quantify $\mathrm{CMRO}_{2}$ which would access to an extremely important physiological parameter in vivo. An MRI-based method allows for easier patient handling and can be used in studies in cohorts of healthy volunteers due to an absence of radioactivity and non-invasive measurement. This can be achieved by one of two methods: direct detection of ${ }^{17} \mathrm{O}$, or indirect detection by virtue of the contrast agent characteristics of ${ }^{17} \mathrm{O}$. The direct measurement entails inhalation of ${ }^{17} \mathrm{O}$ while following the signal curve of directly detected ${ }^{17} \mathrm{O}$ signal. The indirect method is an alternative whereby quantitative mapping of the transverse relaxation time $\left(\mathrm{T}_{2}\right)$ of venous blood only in proton $\left({ }^{1} \mathrm{H}\right) \mathrm{MRI}$ yields the desired $\mathrm{CMRO}_{2}$ values.

The first method should, preferably, be applied on ultra-high field MRI systems such as $7 \mathrm{~T}$ or above and it is a direct measure of metabolic processes in the human body. The accuracy of $\mathrm{CMRO}_{2}$ values based on the ${ }^{17} \mathrm{O}$ signal is dependent on the quantitative knowledge of absolute water content in the human brain (see Chapter 3 ). Previous methods used tissue mass calculated by means of sodium MRI of white and grey matter which is an indirect measure and is confounded by the fact that all the ${ }^{23} \mathrm{Na}$ signal is not captured due to short relaxation times and this potentially leads to inaccurate quantitative results in pathologies with changed water contents. For quantification of absolute water content at fields higher than $1.5 \mathrm{~T}$, methods which were originally used on lower field scanners had to be adapted overcome quantification inaccuracies caused by RF field inhomogeneities [4-11]. Based on a well-known correlation between tissue $T_{1}$ and proton density (PD), a new correction method was developed to estimate the receive bias field properly; Chapter 3, more precisely section 3.3, provides a detailed description thereof. Quantitative water content determination was 
performed on healthy volunteers and the results are shown in Chapter 4. Natural abundance images of the ${ }^{17} \mathrm{O}$ signal at $9.4 \mathrm{~T}$ ultra-high field strength are the basis for further studies of $\mathrm{CMRO}_{2}$ quantification. These measurements are covered in detail in section 3.1.2 with results shown in section 4.2.1

The second method for quantification of $\mathrm{CMRO}_{2}$ based on changes of $\mathrm{T}_{2}$ relaxation rates of venous blood can be performed at clinical field strengths such as $3 \mathrm{~T}$ and does not need additional breathing systems, leading to easier patient handling and higher acceptance rates. Yet, it is an indirect method suffering from long acquisition times and error propagation, especially if quantitative $T_{2}$ values are inaccurate. Both methods are described in Chapter 3. An imaging sequence called QUIXOTIC, which was published by Bolar et al. [12-14], has been implemented. Further developments which allow faster image acquisitions at higher resolutions, are less prone to stimulated echo effects, and at the same time give rise to clinically acceptable measurement times were introduced into the QUIXOTIC sequence. This work is described in section 3.1.4 and following subsections. Stimulated echo effects could possibly lead to inaccurate $T_{2}$ values and thus to inaccurate $\mathrm{CMRO}_{2}$ values. The modified QUIXOTIC sequence was tested in phantom experiments to determine the accuracy of $T_{2}$ estimation and in vivo on four healthy volunteers to demonstrate the feasibility of fast and accurate $\mathrm{CMRO}_{2}$ estimation. Direct ${ }^{17} \mathrm{O} \mathrm{MRI}$ natural abundance images at an ultra-high field of 9.4 $\mathrm{T}$ were also acquired. The results from both methods are shown in Chapter 4. In Chapter 5 a summary and a discussion of the achieved results and an outlook to further development steps and experiments is provided. 


\section{Chapter 2}

\section{Theoretical Background}

The following sections are designed to present a brief description of the basic physical principles of nuclear magnetic resonance (NMR) and concepts of magnetic resonance imaging (MRI). In addition, an elementary imaging sequence is described. Each section is incipient with a brief overview following are more detailed physical description.

\subsection{Hardware - Components of an MR Scanner}

The MRI scanner is an imaging machine based on the combination of a static magnetic field, switchable magnetic gradient fields, an RF transmitter, and an RF detector (which can be the same), signal digitisation hardware and a computer system for image reconstruction, post-processing, and data storage. The static field is in general very strong (0.2-11.7 T for humans) and generates the macroscopic spin polarisation. The spin polarisation can be manipulated with RF pulses and encoded with spatially varying magnetic fields (commonly termed gradients). The main components are described in more detail below.

\subsubsection{Superconducting Magnet}

High magnetic fields (0.2-11.7 T, usually $1.5-3 \mathrm{~T}$ in clinical applications) with sufficient homogeneity can be achieved with the use of large electric currents in a coil. The simplest example, and one that is not very far from real magnet design, is a solenoid coil with many turns. To be able to use strong electric currents without prohibitive losses due to heating, the resistance of the coil has to be minimised. This is possible if superconducting materials such as niobium-titanium are used. If niobium-titanium is placed in liquid helium at $4.2 \mathrm{~K} \approx-269^{\circ} \mathrm{C}$ the material is a superconductor. Since it is not possible to thermally insulate the liquid helium completely, there is resultant boil-off and the necessity of periodic helium refilling. This contributes to the relatively high operational costs of MRI. More 
modern MR magnets are equipped with so-called cold-head systems. Boiling of liquid helium in the magnet takes place at the usual rate, but as the cold gas rises it recondenses on the surface of a cold finger and falls back as liquid. Thus, net helium boil-off is much reduced and the high costs (and technological requirements) for helium refills are transferred to more conventional energy costs for cooling the cold finger.

\subsubsection{Radiofrequency and Magnetic Shielding}

The required high homogeneity of the magnetic field and weak MR signals and thus very sensitive RF coils require a completely shielded room. This is fulfilled with a so-called Faraday shield. All walls, the ceiling and the floor are made from sheet metal. Every electrical signal which is needed in the magnet room has to be routed through specially designed filters or be converted into optical signals outside the magnet room and back into electrical inside the room. This is an absolute necessity to ensure that no external, interfering signals are inadvertently fed into the magnet room. The magnet itself is shielded in order to facilitate a sharper drop off of the external, stray magnetic field. In the past and for so-called ultrahigh-field magnets (e.g. 9.4 T) the magnet room has an inner chamber made from soft iron (passive shielding). Modern clinical magnets are made from two counter-wound coils. These coils produce the desired magnetic field inside the magnet-for example $3 \mathrm{~T}$, but work against each other outside the magnet and thus the stray field reduces over a short distance (active shielding). Actively shielded magnets have the huge advantage of easier siting and saving costs.

\subsubsection{Shimming and Shim Coils}

As mentioned previously, a very homogenous magnetic field is a prerequisite for modern MRI. The homogeneous field from the magnet is itself disturbed by the patient. To compensate for these effects, it is possible to use a set of coils which are integrated into the scanner. These coils have the ability to create an opposing magnetic field which compensates for the inhomogeneities caused by the patient. This process is called "shimming". Modern MRI systems automatically perform shimming before every measurement. For spectroscopy, often shimming is first carried out in automatic mode and is then fine-tuned by hand by an experienced operator. 


\subsubsection{Gradient Coils}

The gradient coils are constructed in such a way that a linear variation of the magnetic field along three different axes is possible. One of these axes is orientated along the main magnetic field ( $z$-axis). The other two axes, $x$ and $y$, are orientated orthogonal to the $z$-axis. MR sequences require precise control of the currents in the gradient coils. The typical noise exposure of an MRI examination is caused by fast switching of current in the gradient coils. Every switching action results in a mechanical force, which causes noise. Gradient amplifiers are a fundamental component to reach the required currents and in the necessarily short rise times $(0-600 \mathrm{~A}$ in $200 \mu \mathrm{s})$. Modern clinical MR scanners are capable of generating gradient fields of up to $70 \mathrm{mT} / \mathrm{m}$ with slew rates of around $200 \mathrm{~T} / \mathrm{m} / \mathrm{s}$. Values higher than these are not really relevant in clinical systems due to safety issues (see section 2.4).

\subsubsection{RF Coils}

RF coils can be subdivided in three main categories: transmit coils; receive coils; and trans$\mathrm{mit} /$ receive coils. Excitation of the spins is achieved with transmit coils and the resulting signal is acquired with receive coils. Some RF coils can be used for both purposes, that is, they are first used to transmit the RF pulse and then to receive the signal. Surface coils are used for localised imaging whereby the decision to use them is based on a compromise between increased sensitivity and reduced volume coverage. Phased array coils are an attempt to have both good sensitivity and good volume coverage; phased array coils comprise a set of surface coils that have been carefully arranged to cover the desired volume. Sophisticated image reconstruction routines are then used to combine the images (high SNR; reduced coverage) acquired by each coil element into one image (high SNR; desired volume coverage). Furthermore, phased array coils are a prerequisite for performing parallel imaging (accelerated imaging methods). The use of powerful RF amplifiers is mandated for the transmit chain and very sensitive pre-amplifiers are required thereafter to amplify the weak MR signal from the receiver coils in the receive chain. RF coils have to be tuned to the correct Larmor frequency of protons. The choice of the RF coil for a given examination is dictated by a number of factors including the MRI sequence that is to be employed. These factors include the following: body area of interest; volume coverage; parallel imaging compatibility; desired SNR; and the diagnostic question. 


\subsection{NMR Phenomenon}

Spin is a quantum-mechanical property of subatomic particles such as protons, neutrons, electrons, etc., and is a physical quantity like mass or electric charge. In accordance with the principles of quantum mechanics, it takes discrete integer or half-integer values (in units of the reduced Planck constant $h / 2 \pi=\hbar$ ). In a macroscopic world, the spin is not commonly observable. Nuclei and particles with a spin number larger than zero have an observable associated magnetic moment. This is proportional to the spin $(\vec{I})$ through a nucleus-specific constant called the gyromagnetic ratio, $\gamma$. The magnetic moment, $\mu$, is given by:

$$
\vec{\mu}=\gamma \vec{I}
$$

Based on this property, the magnetic resonance phenomenon provides a way of detecting the spin of subatomic particles. It is the basis of MRI and will be described in the following.

\subsubsection{Spin, Magnetisation and Larmor Precession}

Hydrogen is the most abundant element in the human body. The nuclei of all three hydrogen isotopes, hydrogen, deuterium, and tritium, have a magnetic moment. By an extremely fortunate coincidence for NMR/MRI and modern medicine, the proton has the largest magnetic moment among all stable nuclei (and tritium the largest among all naturally occurring nuclides). Hydrogen thus holds a special place, since medical applications of MRI are almost exclusively based on proton MRI. In the following, we will first refer to protons only. Other nuclei will be discussed later in section 2.6.

The magnetic moment of a proton placed in an external magnetic field precesses around the axis of the magnetic field at a frequency $\omega$ which is defined by a product of the magnetic flux density, $B_{0}$, and the gyromagnetic ratio, $\gamma$. This frequency is given by:

$$
\omega=\gamma B_{0} .
$$

Equation (2.2) is called Larmor equation and describes the Larmor frequency (frequency of the precession) of the spin in a magnetic field. This phenomenon is represented in a schematic way in Figure 2.1. Frequencies are given in Hertz and the magnetic flux density in Tesla. Therefore, the dimension of $\gamma$ is $\mathrm{Hz} / \mathrm{T}$. For protons $\gamma=42.52 \mathrm{MHz} / \mathrm{T}$. Table 2.1 gives gyromagnetic ratios for different atoms. 


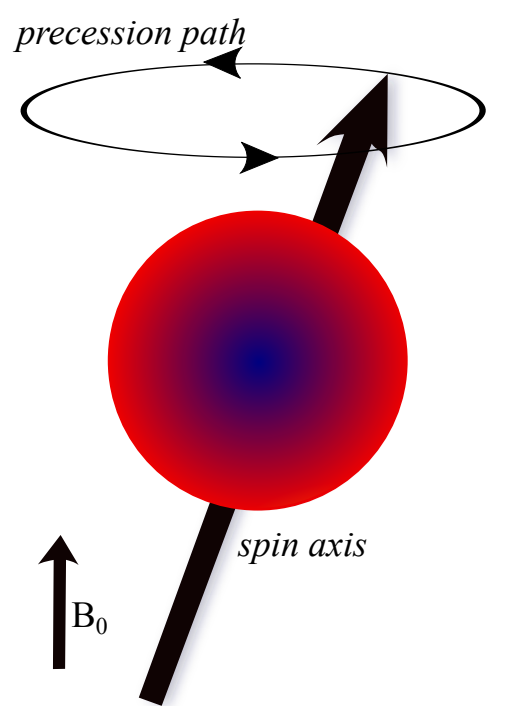

Figure 2.1: Schematic representation of the magnetic moment of a proton and its precession about a magnetic field, $B_{0}$. Adapted from [25].

\subsubsection{Spin}

More detailed, nuclei with an odd number of protons and/or neutrons have a nonzero nuclear spin. NMR can be observed for these elements. The spin angular momentum is given by

$$
\vec{S}=\hbar \hat{I}
$$

where $\hbar$ is Planck's constant divided by $2 \pi$ and $\hat{I}$ is the spin operator. The magnetic dipole moment is defined by

$$
\vec{\mu}=\gamma \vec{S}=\gamma \hbar \hat{I}
$$

where $\gamma$ is the gyromagnetic ratio which is a known constant that differs for different nuclear species. It is noted that the difference between (2.1) and (2.4) is given by the simplified depiction in (2.1) in contrast to the operator based representation of $\hat{I}$ in (2.4). Many different nuclei such as ${ }^{1} \mathrm{H},{ }^{17} \mathrm{O},{ }^{23} \mathrm{Na}$ and ${ }^{31} \mathrm{P}$ can be used for nuclear magnetic resonance. Because ${ }^{1} \mathrm{H}$ is the most abundant nucleus in the human body, magnetic resonance imaging is mainly based upon protons but since ultra-high MRI scanner have become increasingly more available and the first manufacturer has announced clinical approved $7 \mathrm{~T}$ machines, other nuclei are becoming more interesting. 


\begin{tabular}{cccc}
\hline Nucleus & Spin & $\frac{\gamma}{2 \pi}[\mathrm{MHz} / \mathrm{T}]$ & Abundance in human body \\
\hline${ }^{1} \mathrm{H}$ & $1 / 2$ & 42.575 & $88 \mathrm{M}$ \\
${ }^{19} \mathrm{~F}$ & $1 / 2$ & 40.054 & $4 \mu \mathrm{M}$ \\
${ }^{23} \mathrm{Na}$ & $3 / 2$ & 11.262 & $80 \mathrm{mM}$ \\
${ }^{31} \mathrm{P}$ & $1 / 2$ & 17.235 & $75 \mathrm{mM}$ \\
${ }^{17} \mathrm{O}$ & $5 / 2$ & -5.77 & $16 \mathrm{mM}$ \\
\hline
\end{tabular}

Table 2.1: Gyromagnetic ratios and abundances for various nuclei in the human body [26]

The natural abundance is given in $1 \mathrm{M}=1 \mathrm{molar}=1 \mathrm{~mole} /$ litre. For comparison, the ${ }^{1} \mathrm{H}$ molarity of water is $110 \mathrm{M}$. If we assume a water content of about $80 \%$ for brain grey matter, this leads to an abundance of $88 \mathrm{M}$. In case of ${ }^{17} \mathrm{O}$, a negative gyromagnetic ratio can be observed. This is due to the fact that the magnetic moment is anti-parallel to the angular momentum vector.

\subsubsection{Interactions with a Static Magnetic Field}

First a simple depiction of the spin interactions in the presence of a static magnetic field is given. Following this a more detailed view can be found in section 2.2.1.2.1.

Due to the interaction of the magnetic moment of the nucleus (spin of $\frac{1}{2}$ ) with the magnetic field, two energy levels appear: the state with the spin parallel to the field is lower in energy than the state with the antiparallel spin. A significant number of the spins thus orient themselves parallel to the field. The energy difference between the two levels is characterised by the Larmor frequency, and is very small in comparison with the atomic scale of energy ( $\mathrm{eV}=$ electron Volts) or the nuclear one $(\mathrm{MeV})$. At a field of $1.5 \mathrm{~T}$, this energy is approximately $0.3 \cdot 10^{-6} \mathrm{eV}$ and much smaller than the Brownian motion (0.025 $\mathrm{eV}$ at room temperature). The polarisation of protons in a magnetic field is correspondingly small, with a net effect of $\approx 10^{-5}$ (the spins of 10 protons in each million are parallel to the field) at a field of $1 \mathrm{~T}$ and room temperature. Resonant absorption of electromagnetic energy by the nuclei in a magnetic field at the Larmor frequency is associated with the change of the orientation of the magnetic moment (from parallel to the field to antiparallel). A quantum mechanical description gives an exact explanation of all effects, but in most cases a classical description suffices. In this reduced view, not every single spin but a group of spins precessing at the same local frequency (isochromat) is the basis unit of the description. This group is described by the sum of all magnetic effects of neighbouring spins and an effective magnetic momentum, $M_{0}$, can be calculated via a vector sum. 
The following Sections 2.2.1.2.1 and 2.2.1.2.2 gives a more detailed description of the mentioned interactions. The resonant emission or absorption of electromagnetic signal in a classical framework considering the macroscopic magnetisation is described later in section 2.2.2.

\subsection{Magnetisation}

Classically, in the presence of a static magnetic field, $\overrightarrow{B_{0}}$, in the $z$ direction the spins align along the direction of $\overrightarrow{B_{0}}$ which gives rise to a net magnetisation moment, $\vec{M}$.

The potential energy, $E$, of a magnetic moment $\vec{\mu}$ in the presence of a magnetic field, $\vec{B}$, is given by

$$
E=-\vec{\mu} \cdot \vec{B}
$$

and in the case of a static magnetic field $B_{0}$ in the longitudinal $z$ direction

$$
E=-\gamma S_{z} B_{0}
$$

$S_{z}$ is quantised to $\gamma I_{z}$ which is $I_{z}=m \hbar, m \in-i,-i+1, \ldots,+i$ and in the case of hydrogen $I_{z}= \pm \frac{1}{2}$. This behaviour is the so-called Zeeman effect

$$
E(m)=-\gamma m \hbar B_{z}
$$

For proton imaging this leads to one parallel population, $n_{+}$and one antiparallel population, $n_{-}$energy state with

$$
\Delta E=\gamma \hbar B_{0}
$$

where the parallel state is the state with the lower energy. Due to the dependency of the thermal energy on the magnetic field interactions this leads to the ratio

$$
\frac{n_{-}}{n_{+}}=\exp \left(-\frac{\Delta E}{k_{B} T}\right) \approx 0.99998
$$

at $T=300 \mathrm{~K}$ and $B_{0}=3 T$. 


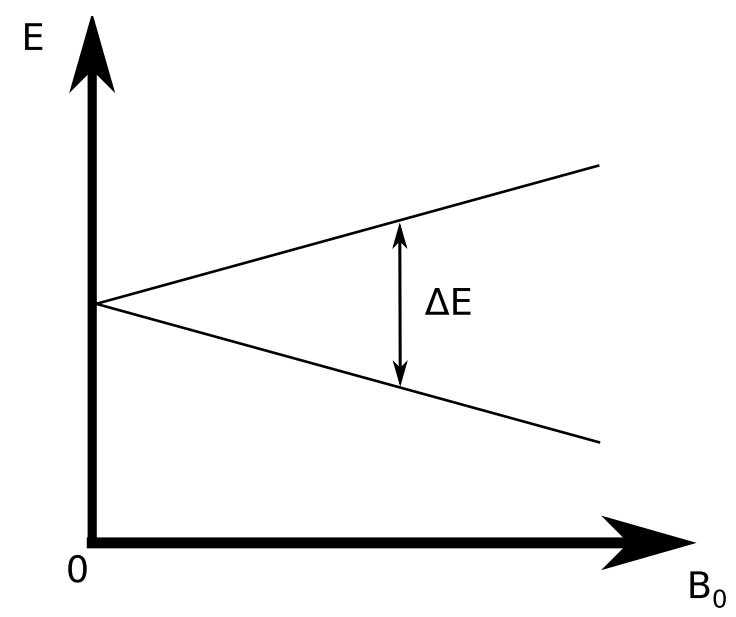

Figure 2.2: Zeeman splitting of proton energy states under influence of a magnetic field $B_{0}$.

This small ratio leads to a low signal to noise ratio (SNR). A commonly used definition of the SNR is given by

$$
\mathrm{SNR}=\frac{S_{\mathrm{ROI}}}{\sigma\left(S_{\text {noise }}\right)}
$$

where $S_{\mathrm{ROI}}$ is the mean signal in a region of interest (ROI) and $\sigma\left(S_{\text {noise }}\right)$ the standard deviation of noise [27].

The higher the field strength of the scanner, the larger the polarisation between the two energy states and thus the SNR increases. This indicates the importance of ultra-high field MRI. In general, the equilibrium nuclear magnetisation, $M_{0}$, is given by [27]

$$
M_{0}=\frac{N \gamma^{2} \hbar^{2} I(I+1) B_{0}}{3 k_{B} T}
$$

with $N$ equals the number of nuclear spins per unit volume, $\hbar$ is Planck's constant, $k_{B}$ is Boltzmann's constant and $T$ is the temperature. Since the temperature can't be changed there is only one variable - $B_{0}$ - left to increase the equilibrium nuclear magnetisation and gain a better SNR.

\subsection{Precession}

If the system is at thermal equilibrium, the magnetisation, $\vec{M}=\sum \vec{\mu}$ and $\vec{B}$ are pointing in the same direction. If the thermal equilibrium is disturbed and $\vec{M}$ and $\vec{B}$ do not point in the same direction, a torque causes a precessional behaviour of the magnetisation. This dynamics is described by

$$
\frac{\mathrm{d} \vec{S}}{\mathrm{~d} t}=\vec{\mu} \times \vec{B}
$$


Multiplying both sides with $\gamma$ and summing over the unit volume results in

$$
\frac{\mathrm{d} \vec{M}}{\mathrm{~d} t}=\vec{M} \times \gamma \vec{B}
$$

Equation (2.13) describes a precession of $\vec{M}$ about $\vec{B}$ with a frequency of

$$
\begin{aligned}
& \omega=\gamma B\left[\frac{\mathrm{rad}}{\mathrm{s}}\right] \\
& f=\frac{\gamma}{2 \pi} B[\mathrm{~Hz}] .
\end{aligned}
$$

This well defined resonance frequency is the so called Larmor frequency. Every spin rotates with this frequency with respect to the inertial laboratory frame. As noted in chapter 2.3.1.2.2, a transformation into a rotating frame of reference simplifies calculations.

\subsubsection{Resonance: RF Excitation and Free Induction Decay}

When a sample containing protons is placed inside a magnetic field, thermal equilibrium is established within a characteristic time and the distribution of spins among the available energy levels follows a Boltzmann distribution given by

$$
N(E)=N_{0} \exp \left(-\frac{E}{k_{B} T}\right)
$$

where $E=\gamma m \hbar B ; m= \pm \frac{1}{2}, \frac{1}{2}$. A macroscopic magnetisation is thus established, since the number of protons with spins parallel to the field (lower energy) is larger than the number of protons with spins antiparallel to the field (higher energy). This equilibrium can be perturbed by applying an on-resonance electromagnetic radiofrequency pulse (RF pulse).

In order for resonant absorption to take place, the frequency of the pulse has to match the Larmor frequency which is a quantum-mechanical phenomenon. An energy quantum of the RF field, a photon, induces a transition between a state of low energy (spin up) and a state of high energy (spin down). The projection of the spin on the axis defined by the magnetic field changes by $\hbar$ and needs to be compensated by the photon. Only photons propagating along the axis of the magnetic field with negative helicity (left-hand circular polarisation) and thus a projection of the spin of the axis of propagation of $\hbar$ will be absorbed. Since the magnetic and electric fields are perpendicular to the direction of propagation, the RF field has to be perpendicular to $B_{0}$ in order to produce an effect.

The remaining process can be described classically. The amplitude of the RF pulse is small when compared to the static $B_{0}$-field. 


\subsubsection{Interaction with a Radiofrequency Field}

The spins are rotated from the $z$-axis (along the direction of the static magnetic field) towards the $x y$-plane with a well-defined flip angle, $\alpha$, which is related to the duration and amplitude of the RF pulse. The macroscopic magnetisation, $M_{0}$, is tilted at the end of the pulse, oriented at an angle $\alpha$ with respect to the external static $\mathrm{B}_{0}$ field, and precesses around the $z$-axis. This behaviour is shown in Figure 2.3.

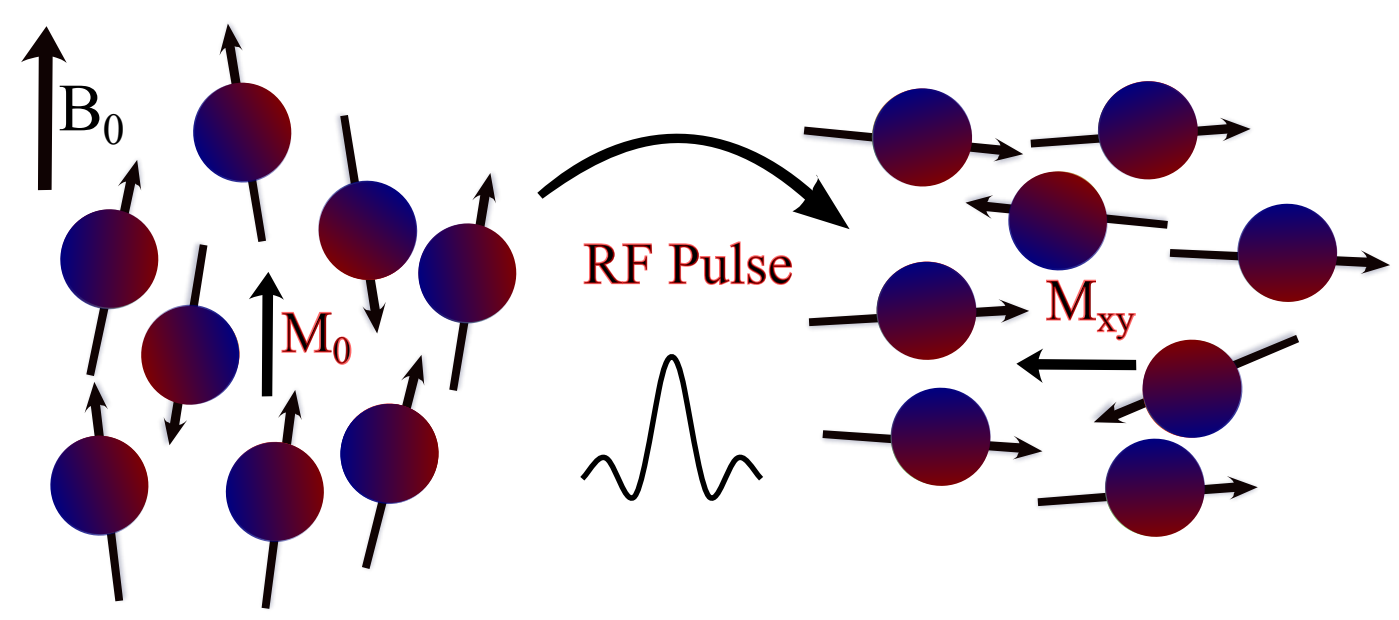

Figure 2.3: Classical representation of the interaction of spins with a $90^{\circ} \mathrm{RF}$ pulse producing spin rotation. This leads to a macroscopic detectable magnetisation, $M_{x y}$, perpendicular to the static field $B_{0}$. Adapted from [25].

Subsequently, the magnetisation along the $z$-axis regrows towards its equilibrium value of $M_{0}$ and simultaneously the transverse magnetisation $M_{x y}$ decays towards its equilibrium value of zero. These are two independent processes and the magnitude of the magnetisation (modulus of $M_{z}+M_{x y}$ ) is not conserved. During this process, electromagnetic waves (the measured signal) are emitted at the Larmor frequency. They can be detected with a coil via the voltage induced by the oscillating field, which is the same principle as a bicycle dynamo. The measured signal is proportional to $M_{x y}$. The evolution of the signal can be varied via MR sequence parameters which influence for example additional RF pulses or so-called crusher gradients. This gives rise to the huge number of options to vary the image contrasts and will be explained in the next section.

Usually, the magnetisation is tipped out of equilibrium (called excitation) by a radiofrequency field, $\overrightarrow{B_{1}}$, which is applied in a direction perpendicular to the $B_{0}$ field and rotating with the resonant frequency. In a classical view, $\overrightarrow{B_{1}}$ induces a torque on the magnetisation which implies a rotation out of the equilibrium position by some well-defined angle. In the quantum mechanical description, the applied RF energy at Larmor frequency stimulates 
an energy exchange between the two energy populations. This explanation only describes the behaviour of the longitudinal component of the magnetisation but it does not describe the behaviour of the transverse component. A classical description is sufficient and will be used to described the behaviour of the transverse component. Directly after excitation, the magnetisation precesses about the $z$ axis. The precessional frequency is proportional to the applied field. Via Faraday's law of induction, it is possible to detect the transverse component of the magnetisation. To detect the signal, the same RF coil as that for transmission of the $\overrightarrow{B_{1}}$ field can be used. A change of the flux, $\Phi$, which is caused by the precessing magnetisation during a time, $t$, induces an electromotive force (EMF) $\epsilon$

$$
\epsilon=-\frac{\partial \Phi}{\partial t},
$$

and is called free induction decay (FID). This represents the basic MR signal. A more detailed description can be found in section 2.3.1. 


\subsubsection{Spin Density, Relaxation, Saturation and MR Contrast}

Medical imaging using computer tomography (CT) depicts tissue contrast based on the absorption coefficients of $x$-rays. In contrast, MRI offers a much more flexible way of contrast generation using different tissue parameters. The main parameters are spin/proton density, $\rho$, longitudinal relaxation time, $T_{1}$, and the transverse relaxation times, $T_{2}$ and $T_{2}^{*}$. While proton density reflects the number of protons in the tissue, the relaxation times depict the "mobility" of the molecules in the tissue or the quantum mechanical interactions of the protons with their environment. All these relaxation processes can be described by an exponential time dependency.

Following an RF pulse, $T_{1}$ reflects the time after which $\frac{1}{e}(\approx 63 \%)$ of the longitudinal magnetisation $M_{0}$ has recovered. During this process, energy is exchanged between the spins and the "lattice"; hence the name spin-lattice relaxation. All magnetisation, which is not aligned parallel to the static field undergoes $T_{1}$ relaxation.

Due to large differences in $T_{1}$ values of white and grey matter (typically a factor 2 ), $T_{1}$ weighted images of the brain can have very high contrast. The $T_{1}$ value is relevant to all MR acquisitions. The entire macroscopic magnetisation can be used a second time, by excitation with a $90^{\circ} \mathrm{RF}$ pulse, resulting in the maximum signal intensity, only after full $T_{1}$ recovery. Based on the fact that a standard imaging sequence utilises many RF pulses to acquire an image, the time between the pulses needs to be long enough to allow for sufficient signal recovery. If the so-called repetition time, TR, is too short, only a part of the longitudinal magnetisation has recovered and therefore the MR signal is weak. This is called $T_{1}$ saturation and its effect needs to be considered for every MRI acquisition. Acquisitions of $T_{1}$-weighted images use this effect and do not allow for full recovery. Based on this, tissues with short $T_{1}$ values are saturated less and appear brighter in images than tissues with a long $T_{1}$ relaxation times.

The transverse magnetisation component, $\vec{M}_{x y}$, experiences an additional relaxation mechanism in which the phase coherence between the spins is increasingly lost due to interactions between protons. This relaxation process is also called spin-spin relaxation. Measurable magnetisation $\vec{M}$ (vector sum of $\vec{M}_{z}$ and $\vec{M}_{x y}$ ) is depicted in Figure 2.4 


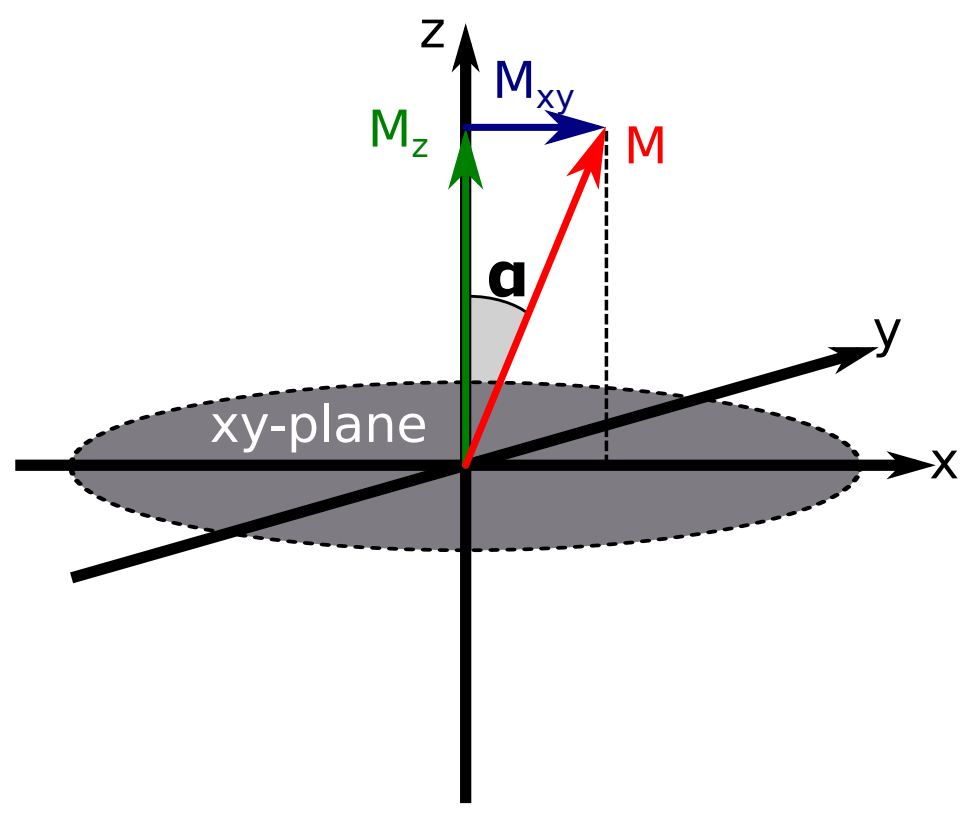

Figure 2.4: Schematic depiction of the magnetisation vector after applying a RF pulse. The transverse part, $M_{x y}$, of the magnetisation vector, which is created by the application of the RF pulse, can be measured. Adapted from [25].

The transverse relaxation time constant, $T_{2}$, reflects the time after the RF pulse at which the initial transverse magnetisation is decreased due to relaxation processes to $\frac{1}{e}$ of its initial value.

Dephasing of the spins in the transverse plane can have additional causes. To reach a synchronous precession, a very homogeneous static magnetic field is needed, otherwise the Larmor frequencies of spins at different positions would differ. Perfect homogeneity is not attainable due to technical issues of building high-field magnets and because of the sample to be measured will itself disturb the homogeneity of the field. Local changes in the magnetic field caused by the sample itself can be large at locations where the magnetic attributes of the sample change significantly, such as at interfaces between different tissues (e.g. bone-soft tissue or soft tissue-air). At these positions the dephasing of the spins is accelerated - the time constant of the exponential decay is called $T_{2}^{*}$ and is always smaller than $T_{2} . T_{2}^{*}$-weighted images are sensitive to magnetic properties of the sample.

Figure 2.5a shows a possible pathway of the magnetisation after excitation in the laboratory frame. Figure 2.5b shows typical MR relaxation behaviours signal of the transverse magnetisation $M_{x y}$ and the longitudinal magnetisation $M_{z}$. As mentioned, the transverse magnetisation relaxes in two different ways. Theoretically and in spin echo experiments with $T_{2}$ and effectively with $T_{2}^{*}$. 


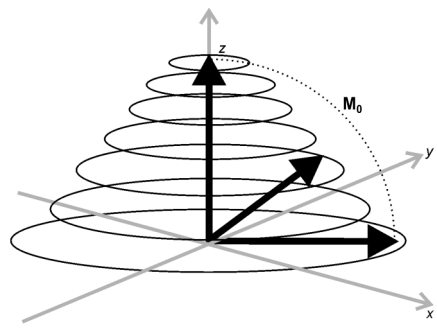

a)

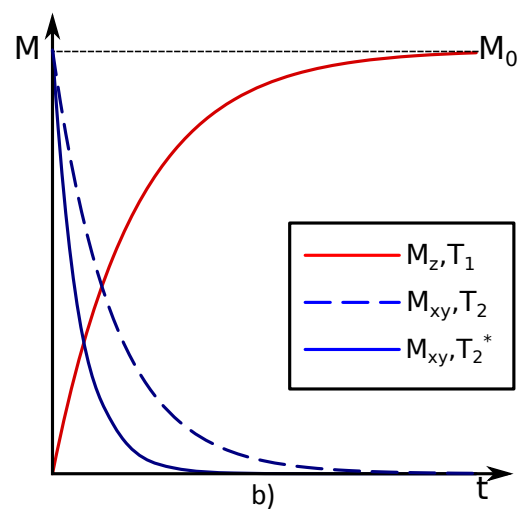

Figure 2.5: Schematic depiction of the magnetisation vector after applying a RF pulse. The transverse part, $M_{x y}$, of the magnetisation vector, which is created by the application of the RF pulse, can be measured.

\subsubsection{Mathematical description of relaxation processes}

Immediately after excitation, the transverse component of the magnetisation decays while the longitudinal part returns to equilibrium. Here, only magnetic dipole-dipole interactions are considered because it is the only dominant mechanism in biological tissues [27].

\subsection{Longitudinal Spin-Lattice Relaxation}

The longitudinal component of the magnetisation can be described by

$$
\frac{\mathrm{d} M_{z}}{\mathrm{~d} t}=-\frac{M_{z}-M_{0}}{T_{1}}
$$

and has the solution [27]

$$
M_{z}=M_{0}+\left(M_{z}(0)-M_{0}\right) \exp \left(-\frac{t}{T_{1}}\right) .
$$

The return of the longitudinal component of the magnetisation to equilibrium is welldescribed by the spin-lattice time constant, $T_{1}$. Equation (2.19) describes an exchange of energy between the nuclei and the surrounding lattice. If $M_{z}$ is completely restored to $M_{0}$ then thermal equilibrium is established.

Randomly fluctuating microscopic magnetic fields at the resonance frequency of the spins, decrease $T_{1}$ because of inducing transitions between the two states $n_{+}$and $n_{-}$. This results in a field dependence and $T_{1}$ increases with increasing $B_{0}$ [27]. 


\subsection{Transverse Spin-Spin Relaxation}

Relaxation of the transverse component of the magnetisation can be described by [27]

$$
\frac{\mathrm{d} M_{x y}}{\mathrm{~d} t}=-\frac{M_{x y}}{T_{2}}
$$

Following a $90^{\circ}$ excitation the solution of equation (2.20) is simply

$$
M_{x y}=M_{0} \exp \left(\frac{-t}{T_{2}}\right)
$$

where $T_{2}$ represents the spin-spin time constant, which characterises the decay of the transverse magnetisation. The same field fluctuations, as mentioned in section 2.2.3.1.1, take effect for $T_{2}$ relaxation. Also $z$-component field fluctuations affect $T_{2}$ and therefore $T_{2} \leq T_{1}$. The fluctuations of the $z$-component often dominate the $T_{2}$ relaxation process and do not contribute to the $T_{1}$ relaxation process which means that $T_{2}$ is largely independent of the field strength.

\subsection{Bloch Equation}

Combining equations (2.13),(2.18) and (2.20), this results in the Bloch equation, which describes the dynamics of nuclear magnetisation phenomenologically:

$$
\frac{\mathrm{d} M}{\mathrm{~d} t}=\underbrace{\vec{M} \times \gamma \vec{B}}_{\text {precession }}-\underbrace{\frac{M_{x} \overrightarrow{e_{x}}+M_{y} \overrightarrow{e_{y}}}{T_{2}}}_{\text {transverse relaxation }}-\underbrace{\frac{\left(M_{z}-M_{0}\right) \overrightarrow{e_{z}}}{T_{1}}}_{\text {longitudinal relaxation }},
$$

where $\overrightarrow{e_{x}}, \overrightarrow{e_{y}}, \overrightarrow{e_{z}}=$ unit vectors in $x, y, z$ directions. The phenomenological nature of relaxation processes is described by an exponential behaviour. 


\subsection{MR Imaging and MR Sequences}

In the previous sections, the principles of generating an MR signal from all protons in a sample were illustrated. This section introduces elements of imaging with magnetic resonance. An MRI sequence is, in accord with its name, a time sequence of RF and gradient pulses and signal read-out times. The building block of the imaging sequence, which is repeated with slightly varying parameters until the whole image is acquired, is contained in the so-called repetition time, TR. Using an imaging sequence, signals from small volumes, called voxel (a voxel is the 3D equivalent of a 2D pixel) can be separated and reconstructed. This is done by encoding position information into the signal using magnetic field gradients. A spatially varying weak magnetic field-the magnetic field gradient-is thus superposed onto the static magnetic field $B_{0}$ at different time points during an imaging sequence. Due to the linearly varying magnetic field, the distribution of the Larmor frequencies of the protons has a well-defined dependence on the spatial location inside the main magnetic field. In other words, spatial location is encoded into frequency. A Fourier analysis of the measured signal (used to obtain information about the frequencies in a measured signal) gives information about the spatial position of spins. This Fourier analysis is analogous to white light being split into a rainbow of colours by a prism.

\subsubsection{Principles of Magnetic Resonance Imaging}

\subsubsection{1 $k$-space and FT - 2D and 3D}

\subsection{Fourier Transform}

The one-dimensional Fourier Transform (FT) of a function $f(x)$ is

$$
F\left(k_{x}\right)=\int_{-\infty}^{\infty} f(x) e^{-i 2 \pi k_{x} x} \mathrm{~d} x
$$

and is the so-called frequency spectrum of $f(x)$. The inverse 1D Fourier transform to recover $f(x)$ from the frequency spectrum is given by

$$
f(x)=\int_{-\infty}^{\infty} F\left(k_{x}\right) e^{+i 2 \pi k_{x} x} \mathrm{~d} k_{x}
$$

This can be easily extended to 2D and 3D

$$
F\left(k_{x}, k_{y}, k_{z}\right)=\int_{-\infty}^{\infty} \int_{-\infty}^{\infty} \int_{-\infty}^{\infty} f(x, y, z) e^{-i 2 \pi\left(k_{x} x+k_{y} y+k_{z} z\right)} \mathrm{d} x \mathrm{~d} y \mathrm{~d} z .
$$


$x, y$ and $z$ are typically given in units of $\mathrm{mm}$ and thus the unit of $k_{x}, k_{y}$ and $k_{z}$ is cycles $/ \mathrm{mm}$. $k_{x}, k_{y}$ and $k_{z}$ represents the spatial frequency.

It can clearly be seen that it is possible to switch between the measured frequency- or $k$-space and the image-space with the help of these equations. Thus, these equations are of an importance for magnetic resonance imaging and have no analogue in x-ray imaging. In contrast to $x$-ray imaging, the wavelength of the RF signal has no connection to the imaging resolution which in turn is defined by imaging parameters and consequently the applied gradient encoding scheme.

\subsubsection{Selective Excitation and Spatial Encoding}

For illustrative purposes, the above-mentioned principle of gradient encoding can be divided into three independent parts, which usually are applied sequentially.

Initially, a slice-selective RF excitation is created by the use of simultaneous RF pulse and gradient fields. It is used to generate transverse magnetisation $M_{x y}$ only from spins contained in a small and well-defined slice of the whole volume. This is performed using a gradient to modify the Larmor frequencies in such way that only protons contained within the desired slice precess with the right, detected frequency. The range of proton Larmor frequencies outside the slice is far from the main frequency of the RF pulse and the resonance condition is not fulfilled. The expression "main frequency" reflects the fact that the RF pulse does not only have one frequency but a frequency distribution with a well-defined bandwidth, which-together with the strength of the magnetic gradient field-defines the slice thickness. RF excitation is described in more detail in the latter section 2.3.1.2.2.

After slice selection, further spatial encoding in two dimensions has to be performed in order to acquire a 2D image. First a brief overview is given in this section. A more detailed description can be found in section 2.3.1.2.4.

Frequency encoding along an axis of the slice is performed using a magnetic field gradient in-for example - the x-direction. This results in different Larmor frequencies of the signals from protons (isochromats) situated at different positions along the axis. Using Fourier analysis, the MR signal can be separated into components with different frequencies and therefore the intensity of the signal at different positions along the $\mathrm{x}$-axis can be calculated. One more dimension-i.e. the $y$-axis - needs to be encoded. Spatial encoding perpendicular to the frequency encoding axis is usually done by so-called phase encoding. In a typical sequence, phase encoding is applied between slice selection and frequency encoding as follows. The magnetic field gradient on the $y$-axis is switched on for a short time. During the applied gradient, spins with different positions along the $y$-axis will precess with different frequencies. After applying the gradient, the phase $\left(\varphi_{\text {encoding }}(y)=\omega(y) T_{\text {encoding }}\right)$ of these spins shows a well-defined (linear) dependence on the spatial location along the $y$-axis. At 
the time of signal read-out, however, the frequency does not depend on y anymore (it depends on $x$, see above). However, y-position information is still contained in the phase and the intensity of the measured signal, which is a vector sum of all signal elements, reflects this phase distribution. In order to be able to disentangle the y-position information, the procedure (slice selection-phase encoding-frequency encoding) has to be repeated many times with slightly different values of the phase-encoding gradients resulting in different phase shifts and different integrated signals for each measurement. The difference in the phase of the various components may be thought of as reflecting frequency encoding along the $y$-axis; this is analogous to frequency encoding on the $x$-axis with the difference that in the $y$-direction this information was built up over repeated cycles of TR.

Thus, by applying a 2D Fourier transform (2D-FT) to all measured signals along the $x$ - and $y$-axis it is possible to reconstruct a $2 D$ image. The number of voxels in the $y$-direction is defined by the number of phase encoding steps. This number determines the acquisition time of the whole MRI exam as being $\mathrm{N}_{\text {phase }}$. TR. On the other hand, the number of voxels along the $x$-direction is given by the number of sampling points of the MR signal measured during the read-out period. This number defines the sampling rate of the analogue-todigital-converter (ADC), which converts the analogue, measured MR signal to a digital signal. This allows for digital storage and computer-aided signal processing - such as the 2D-FT - to be performed.

The principles of spatial encoding are represented in Figure 2.6. Note that in the above discussion the use of $x$ - and $y$-directions was totally arbitrary. The magnetic field gradients are produced by coils which are labelled as $x, y$, and $z$. The simultaneous use of two gradients will simply produce one gradient, which is a vector sum of the aforementioned components. Using simultaneous switching of gradients on all three gradient axes, it is possible to encode slices with arbitrary orientation. This capability to select oblique and double-oblique slices under computer control and without moving the patient or any part of the scanner is another advantage of MRI over other modalities. 


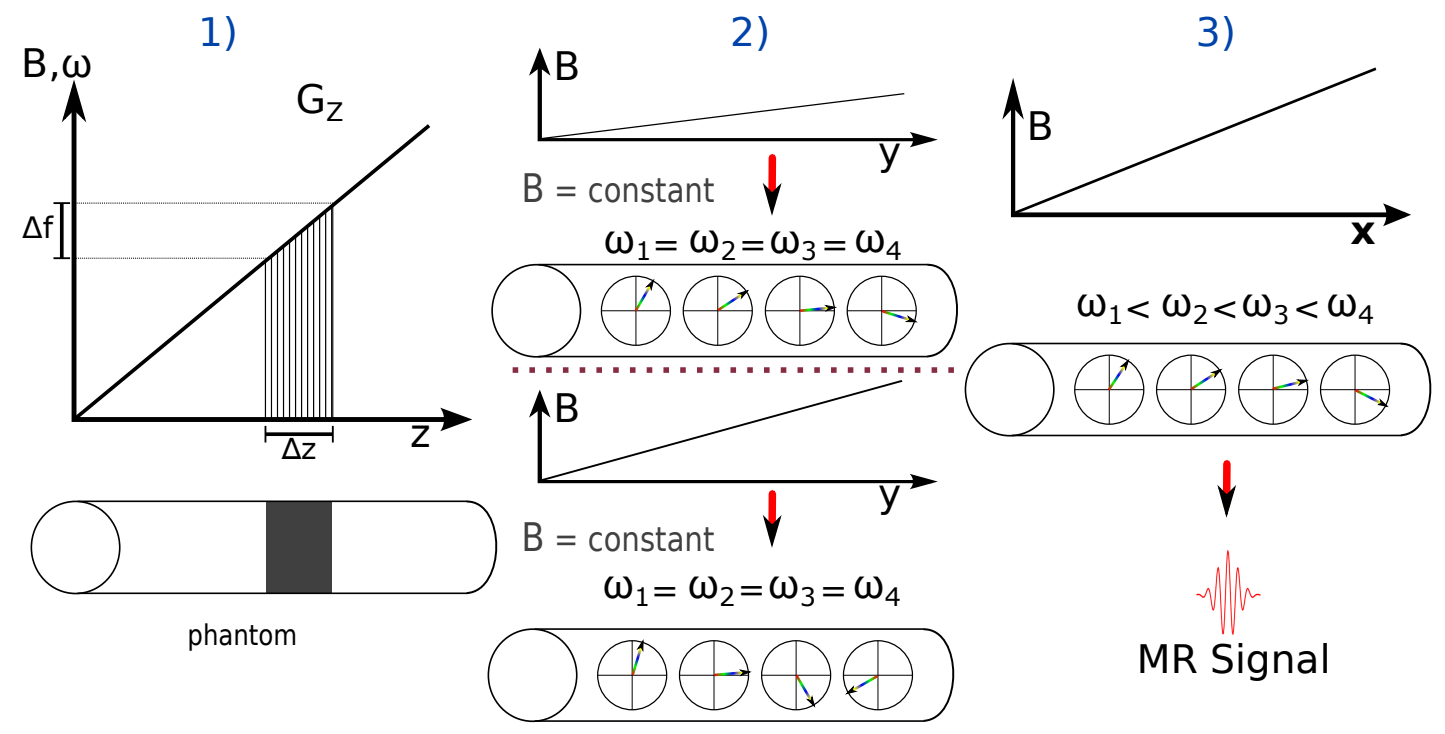

Figure 2.6: Depiction of the principles of spatial encoding. 1) Slice selection: the resonance frequency is varied by the use of a magnetic field gradient in the $z$-direction. Applying a RF-pulse with the right frequency bandwidth only spins in a slice with a thickness $\Delta z$ are excited. 2) Phase encoding: short application of a magnetic field gradient leads to a phase shift of the spin isochromat relative to each other in the direction of the applied gradient (e.g. y). The larger momentum of the applied gradient, the bigger the phase shift. The pase encoding gradient is switched on for a short period of time only. Subsequent the frequency of the spins is the same but the isochromats have different phases. The lower part of part 2) depicts a different phase encoding. 3) Frequency encoding: During acquisition a gradient field is switched on which leads to a spatial dependent frequency on the second orthogonal dimension (e.g. $x$ ). Adapted from [25].

The main goal of the development of new MR pulse sequences is to combine RF pulses and magnetic field gradients in a time efficient way and obtain images with the desired contrast between different types of tissue. A detailed description of the MR physics involved in sequence design can be found in the following Sections and in e.g. Haacke et al. [26].

\subsection{Spatial Resolution and Field-of-View}

\section{Field-of-view}

If restricted sampling takes place in one domain, this leads to replication in the other domain which is the nature of discretised Fourier transformations. As mentioned above, sampling in MRI takes place in the frequency domain. This has to be considered when the field-of-view (FOV) in the object domain is chosen. 


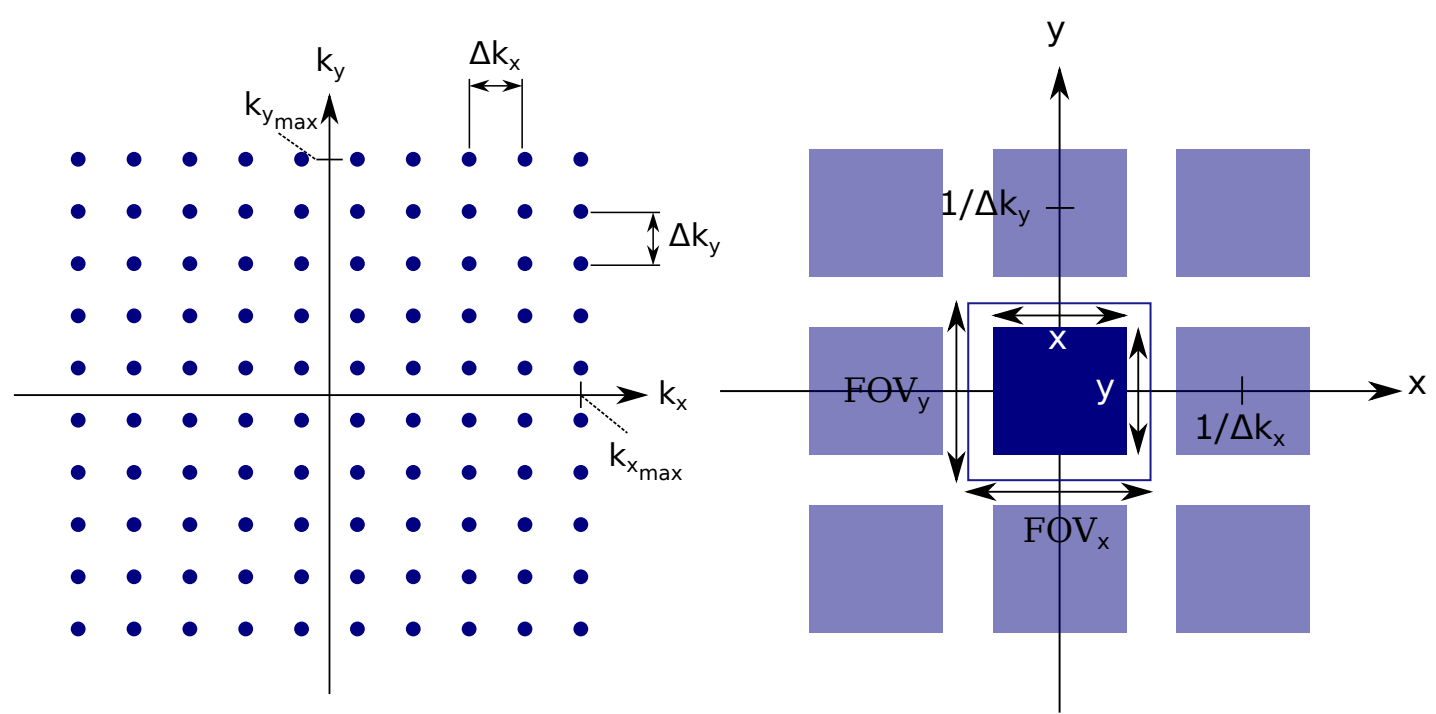

Figure 2.7: Sampling in k-space and corresponging representation in the object domain. Adapted from [27].

From Figure 2.7 one can define the FOV in the 2D imaging case as

$$
\begin{aligned}
\mathrm{FOV}_{x} & =\frac{1}{\Delta_{k_{x}}}=\frac{1}{\frac{\gamma}{2 \pi}\left(G_{x r} \Delta t\right)} \\
\text { and } \mathrm{FOV}_{y} & =\frac{1}{\Delta_{k_{y}}}=\frac{1}{\frac{\gamma}{2 \pi}\left(G_{y i} \tau_{y}\right)}
\end{aligned}
$$

where the readout gradient amplitude $G_{x r}$ is applied during the sampling period $\Delta t$ and the incremental phase encoding gradient amplitude $G_{y i}$ takes place during the time $\tau_{y}$.

\section{Spatial resolution}

As can be seen in Figure 2.7, $k$-space sampling is finite which leads to a 2D sinc blurring function in the object domain. With a given FOV and number of sample points, $N$, the spatial resolution in $x$ and $y$ direction can be defined as

$$
\begin{aligned}
\delta_{x} & =\frac{\mathrm{FOV}_{x}}{N}=\frac{1}{N} \frac{1}{\Delta_{k_{x}}} \\
\text { and } \delta_{y} & =\frac{\mathrm{FOV}_{y}}{N}=\frac{1}{N} \frac{1}{\Delta_{k_{y}}} .
\end{aligned}
$$

While the FOV depends on the $\Delta$ between the sampling period, spatial resolution depends on the widths of $k$-space coverage $k_{\max _{x, y}}$. The latter is defined by the maximum gradient 
areas.

$$
\begin{aligned}
k_{\max _{x}} & =\frac{\gamma}{2 \pi}\left(G_{x r} \frac{\tau_{x}}{2}\right) \\
\text { and } k_{\text {max }} & =\frac{\gamma}{2 \pi}\left(G_{y p} \tau_{y}\right) .
\end{aligned}
$$

Combining Equations $(2.26,2.27,2.30,2.31)$ leads to

$$
\begin{aligned}
\delta_{x} & =\frac{1}{\frac{\gamma}{2 \pi}\left(G_{x r} \tau_{x}\right)} \\
\text { and } \delta_{y} & =\frac{1}{\frac{\gamma}{2 \pi}\left(2 G_{y p} \tau_{y}\right)}
\end{aligned}
$$

if $N$ is large.

\subsection{RF Excitation}

Excitation generally means tipping the magnetisation by some angle out of the equilibrium longitudinal position into a transverse position and can be described by Equation (2.22). If we assume an amplitude modulated $B_{1}(t)$ radiofrequency field which is applied in the transverse direction, the resulting field can be written as

$$
\overrightarrow{B_{1}}(t)=2 B_{1}(t) \cos (\omega t) \overrightarrow{e_{x}}
$$

where $B_{1}(t)$ is an amplitude modulation function and $\omega$ is the carrier frequency which is used for excitation. Equation (2.34) can be decomposed into two circularly polarised fields and be rewritten as

$$
\overrightarrow{B_{1}}(t)=\underbrace{B_{1}(t)\left(\cos (\omega t) \overrightarrow{e_{x}}-\sin (\omega t) \overrightarrow{e_{y}}\right)}_{\text {left-handed field }}+\underbrace{B_{1}(t)\left(\cos (\omega t) \overrightarrow{e_{x}}+\sin (\omega t) \overrightarrow{e_{y}}\right)}_{\text {right-handed field }}
$$




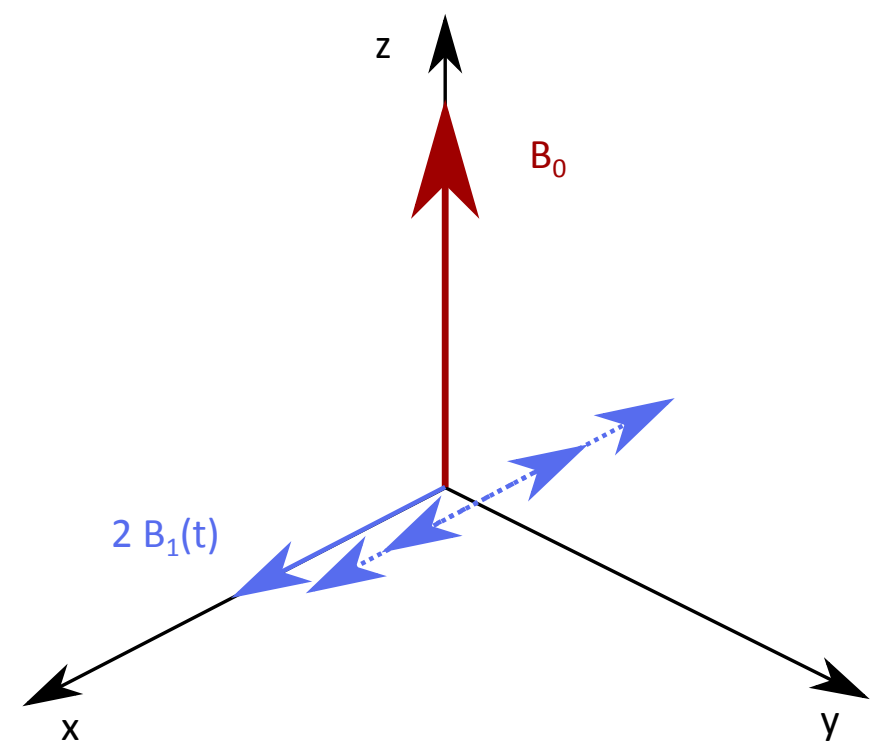

(a) Linearly polarised excitation field applied in transverse plane at frequency $\omega$.
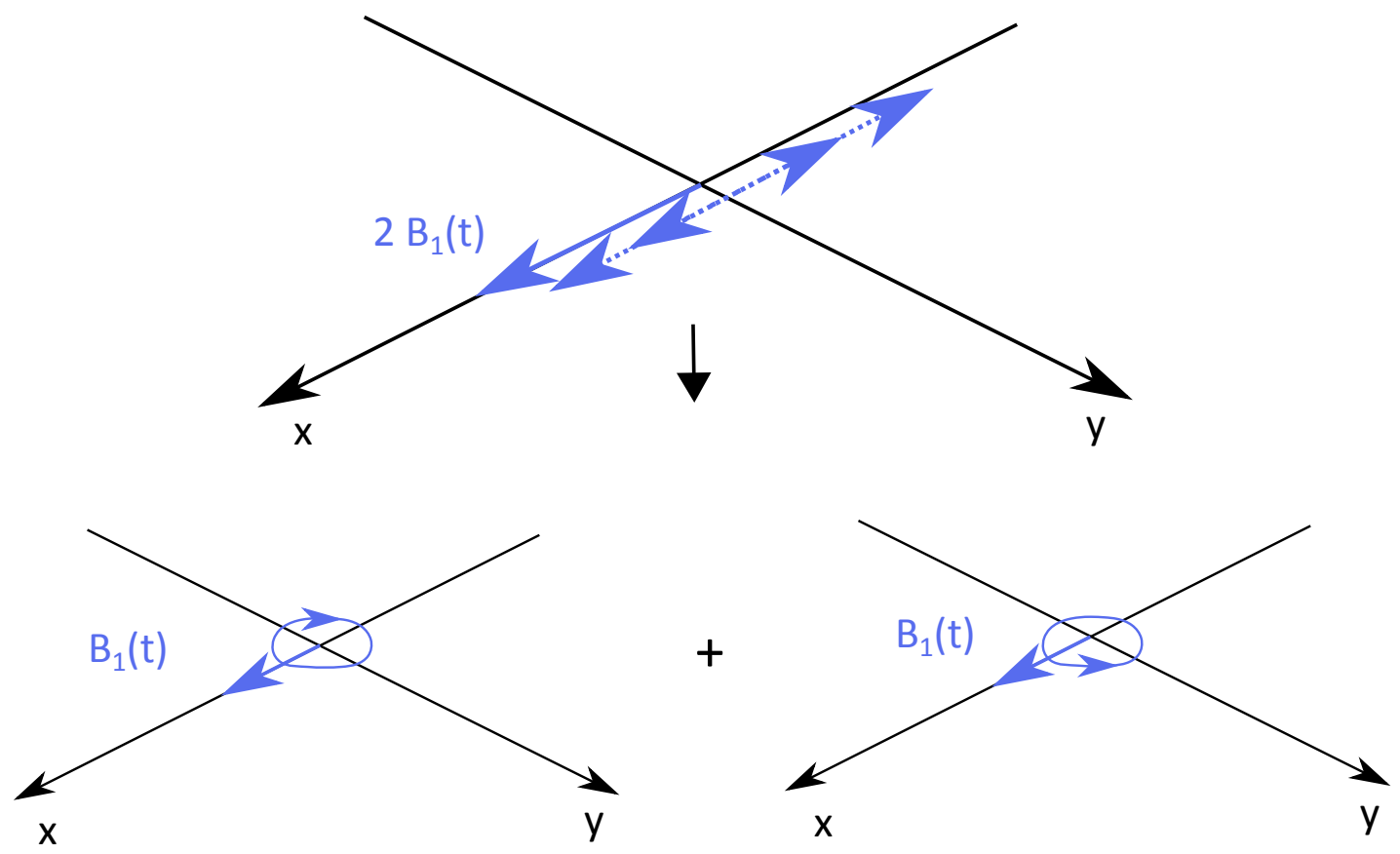

(b) Decomposition of a linearly polarised field into the sum of two counterrotating circularly fields.

Figure 2.8: Linearly polarised field and decomposition into counterrotating circularly polarised fields. Adapted from [27]. 
In contrast to the left-handed field, which has the same rotation direction as the precession and is resonant with the spins, the right-handed field has only a negligible effect on the spins and can be neglected. If we assume a short pulse duration compared to $T_{1}$ and $T_{2}$ we can ignore relaxation terms and the resulting equation can be written as

$$
\overrightarrow{B_{1}}(t)=B_{1}(t)\left\{\cos (\omega t) \overrightarrow{e_{x}}-\sin (\omega t) \overrightarrow{e_{y}}\right\}+B_{0} \overrightarrow{e_{z}}
$$

Therefore we must solve

$$
\left(\begin{array}{l}
\frac{d M_{x}}{d t} \\
\frac{d M_{y}}{d t} \\
\frac{d M_{z}}{d t}
\end{array}\right)=\left(\begin{array}{ccc}
0 & \gamma B_{0} & \gamma B_{1}(t) \sin \omega t \\
-\gamma B_{0} & 0 & \gamma B_{1}(t) \cos \omega t \\
-\gamma B_{1}(t) \sin \omega t & -\gamma B_{1}(t) \cos \omega t & 0
\end{array}\right)\left(\begin{array}{l}
M_{x} \\
M_{y} \\
M_{z}
\end{array}\right) .
$$

Introducing a frame of reference rotating about the $z$-direction at the frequency $\omega$ with

$$
M_{\mathrm{rot}}=\left(\begin{array}{c}
M_{x^{\prime}} \\
M_{y^{\prime}} \\
M_{z^{\prime}}
\end{array}\right), B_{\mathrm{rot}}=\left(\begin{array}{c}
B_{x^{\prime}} \\
B_{y^{\prime}} \\
B_{z^{\prime}}
\end{array}\right)
$$

and

$$
M(t)=M_{\text {rot }}(t) \exp (-i \omega t)
$$

results in

$$
\frac{\mathrm{d} M_{\text {rot }}}{\mathrm{d} t}=M_{\mathrm{rot}} \times \gamma B_{\text {eff }}
$$

In which

$$
\begin{array}{r}
\overrightarrow{B_{\text {eff }}}=\overrightarrow{B_{\text {rot }}}+\frac{\overrightarrow{\omega_{\text {rot }}}}{\gamma} \\
\text { and in turn } \overrightarrow{\omega_{\text {rot }}}=\left(\begin{array}{c}
0 \\
0 \\
-\omega
\end{array}\right)
\end{array}
$$

where a transformation to rotating unit vectors

$$
\begin{array}{r}
\overrightarrow{e_{x}^{\prime}}=\overrightarrow{e_{x}} \cos \omega t-\overrightarrow{e_{y}} \sin \omega t, \\
\text { and } \overrightarrow{{\overrightarrow{e_{y}}}^{\prime}}=\overrightarrow{e_{x}} \sin \omega t+\overrightarrow{e_{y}} \cos \omega t
\end{array}
$$

is introduced in Eq. (2.40). 

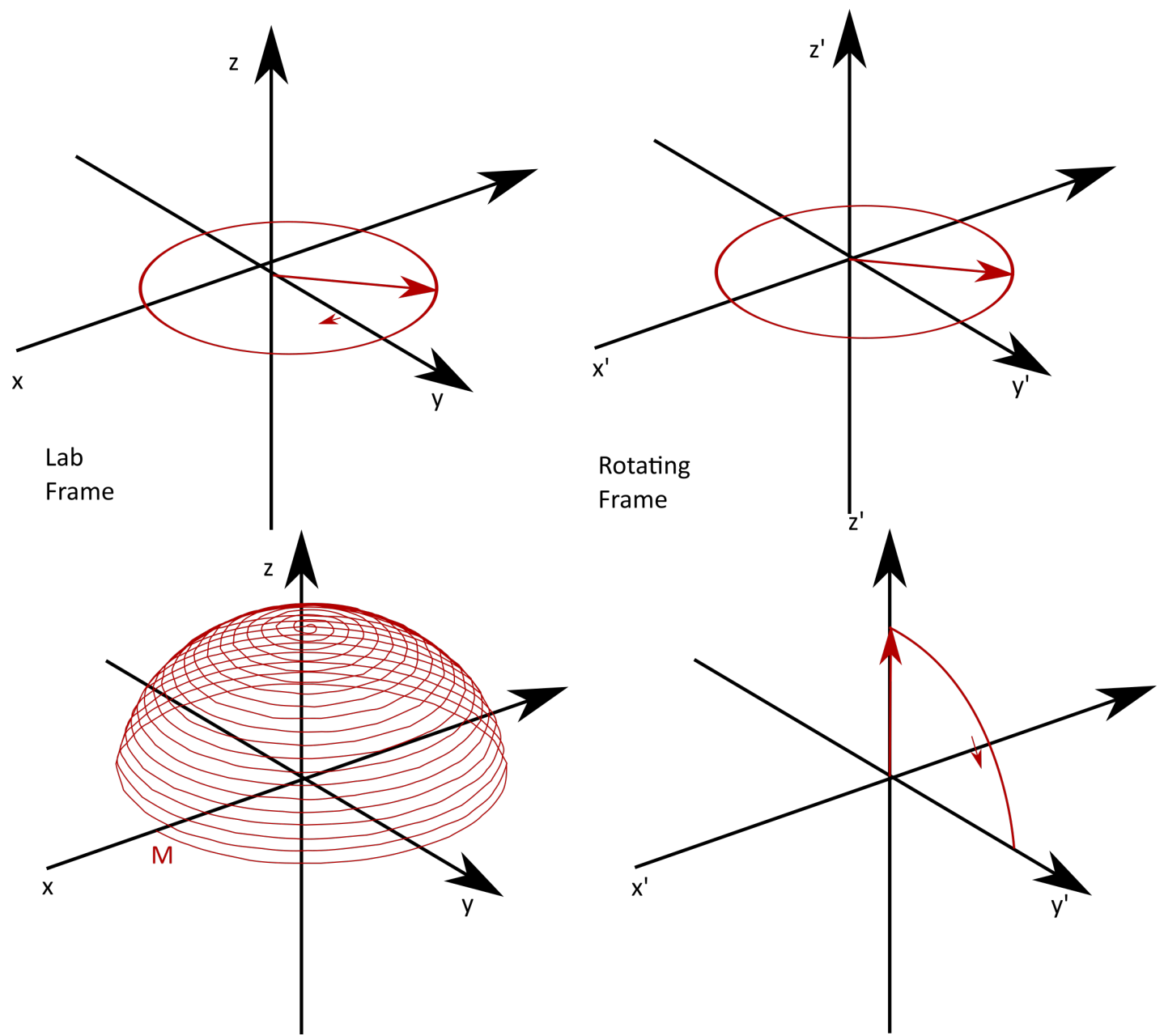

Figure 2.9: Comparison of magnetisation behaviour in laboratory and rotating frames of reference. Adapted from [27].

With Equation (2.40) it is possible to describe nonselective and selective excitation.

\subsection{Free Induction Decay}

The simplest experiment in NMR - if only the acquisition strategy is taken into account - is a so-called Free Induction Decay (FID) experiment. It simply consists of an RF pulse, which creates tranverse magnetisation followed by acquisition of the resulting signal. The signal is sampled in the time-domain and the spectrum of the different Larmor frequencies can be obtained using a Fourier transform (see chapter 2.3.1.1.1) of the acquired signal. Figure 2.10 presents a ${ }^{31} \mathrm{P}$ spectrum which is a Fourier transform of the acquired FID. 


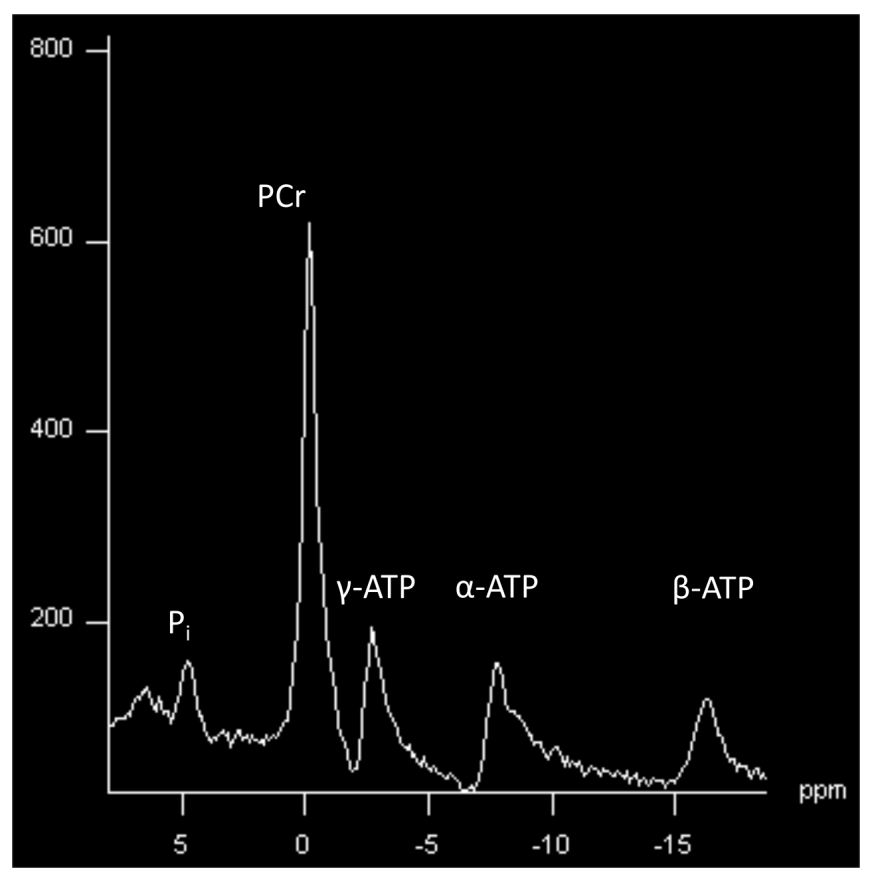

Figure 2.10: real part of FID signal in frequency domain

The measured signal can be described with [27]

$$
S(t)=\int_{x} \int_{y} \int_{z} M_{0}(x, y, z) \exp \left(-\frac{t}{T_{2}}\right) \exp \left(-i \omega_{0} t\right) \exp \left(-i \gamma \int_{0}^{t} G(\tau) \cdot r d \tau\right) d x d y d z
$$

\subsection{Gradient encoding}

Magnetic field gradients are able to change the $B_{\text {eff }}$ field which is exposed to the sample and thus the Larmor frequency can be modulated spatially and temporally. Using these gradients, it is also possible to excite a single slice of the sample in the scanner. Performing so-called frequency and phase encoding of the measured signal, it is feasible to acquire spatial dependent frequencies.

\section{Slice Selection}

Excitation, as it is described in chapter 2.3.1.2.2, excites the entire volume. This enforces the use $3 \mathrm{D}$ readouts as the whole volume contributes to the acquired signal. This can be reduced to a 2D problem, if the size of the excited volume is limited. This is typically achieved with the use of a constant slice selection gradient, which is employed during the RF pulse.

If we assume nonselective on resonance excitation with $\omega=\omega_{0}$ in the case of a general 
$B_{1}(t)$, the angular ratio becomes $\omega_{1}(t)=\gamma B_{1}(t)$. The Bloch equation can be rewritten as

$$
M_{\mathrm{rot}}(t)=\left(\int_{0}^{t} \omega_{1}(s) \mathrm{d} s\right) M_{\mathrm{rot}}(0) .
$$

This results in a tip angle, $\alpha$, of

$$
\alpha=\int_{0}^{\tau} \gamma B_{1}(t) \mathrm{d} t=\int_{0}^{\tau} \omega_{1}(s) \mathrm{d} s
$$

for a general, time-varying $B_{1}(t)$.

If only $B_{0}$ is present, the time varying $B_{1}(t)$ field acts on all spins and the excitation is nonselective. A basic approach to apply selective excitation is the presence of a static magnetic gradient $G_{z}$. In this case $B_{1}(t)$ only affects those spins with Larmor frequency, and therefore the $z$-location matches the bandwidth of the RF pulse. Any other spin with a resonance frequency outside the bandwidth of the applied $B_{1}(t)$ field will remain unaffected. 


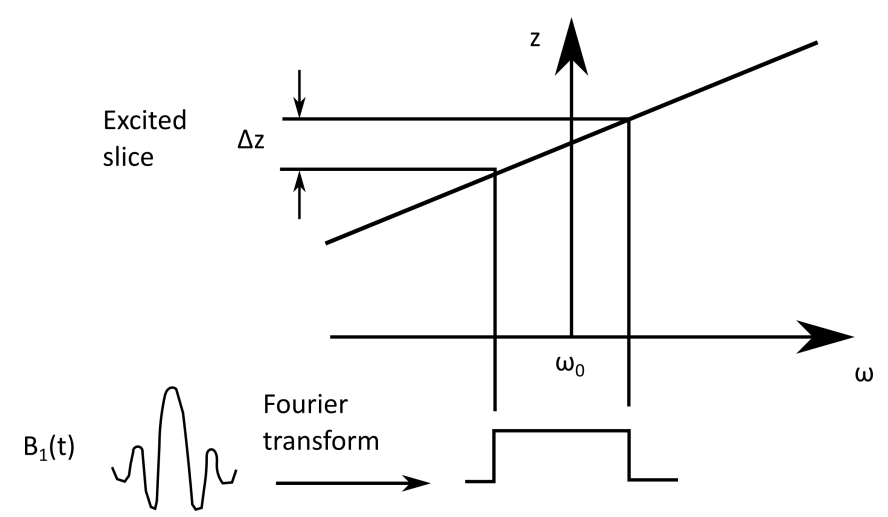

(a) Selective Excitation: $B_{1}$ applied in the presence of $G_{z}$ excites a plane perpendicular to $z$. The Fourier transform of $B_{1}(t)$ approximates the slice profile.
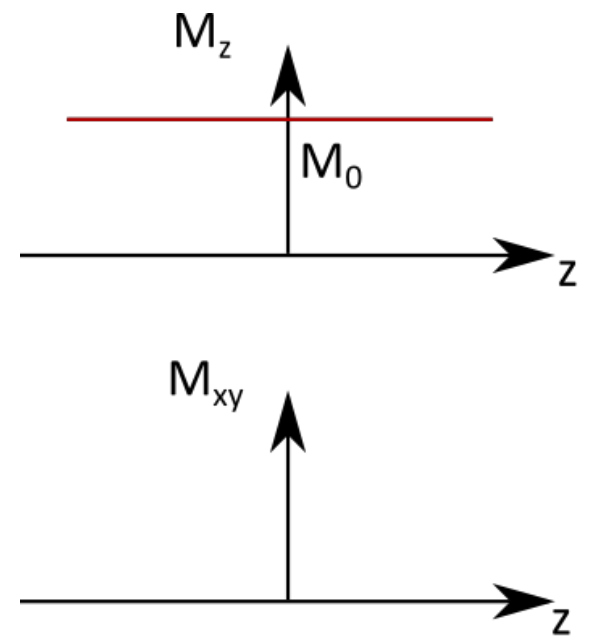

Before excitation
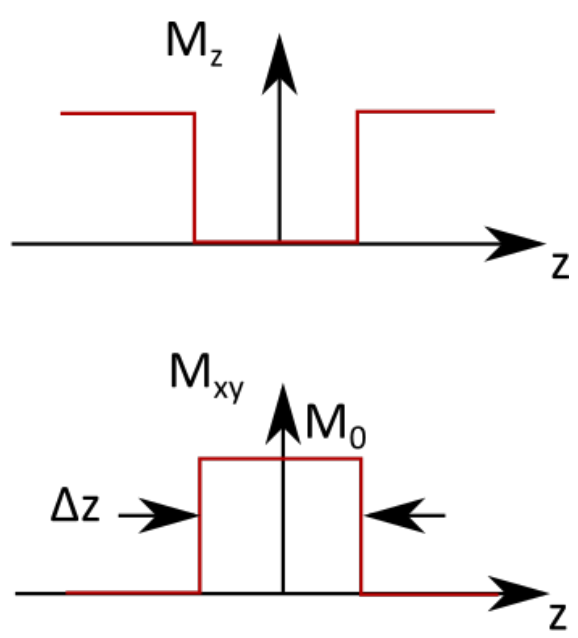

After excitation

(b) Ideal slice profile for a $90^{\circ}$ excitation (slice thickness $=\Delta z$ ).

Figure 2.11: Pictorial description of slice selective excitation. Adapted from [27].

This so-called off-resonant excitation can be described with

$$
\frac{\mathrm{d} M_{\mathrm{rot}}}{\mathrm{d} t}=\left(\begin{array}{ccc}
0 & \omega_{0}+\gamma G_{z} z-\omega & 0 \\
-\left(\omega_{0}+\gamma G_{z} z-\omega\right) & 0 & \omega_{1}(t) \\
0 & -\omega_{1}(t) & 0
\end{array}\right) M_{\mathrm{rot}}
$$

or if the frequency is tuned to the central Larmor frequency

$$
\frac{\mathrm{d} M_{\mathrm{rot}}}{\mathrm{d} t}=\left(\begin{array}{ccc}
0 & \omega(z) & 0 \\
-\omega(z) & 0 & \omega_{1}(t) \\
0 & -\omega_{1}(t) & 0
\end{array}\right) M_{\mathrm{rot}}
$$


In general, the cause of the off-resonant condition is irrelevant. It does not matter if it is caused by an improperly tuned excitation frequency or by a gradient field.

Small tip-angle approximation

Assume that the initial condition at equilibrium equals $M_{\text {rot }}=\left(\begin{array}{c}0 \\ 0 \\ M_{0}\end{array}\right)$ and only small tip angles are applied.

With this so-called small tip-angle approximation, the equations of the transverse components are no longer coupled to the equation of the longitudinal component and the solution is given by

$$
M_{r}(\tau, z)=i M_{0} \exp (-i \omega(z) \tau) \int_{0}^{\tau} \exp (i 2 \pi f(z) s) \omega_{1}(s) \mathrm{d} s .
$$

This can be simplified to

$$
M_{r}(\tau, z=0)=i M_{0} \sin \alpha
$$

If one assumes an excitation exactly on resonance $(z=0)$, the small tip-angle approximation reduces to the nonselective solution. It should be noted that $M_{r}(\tau)$ is imaginary and only a $M_{y^{\prime}}$ component is implied because the magnetisation is rotated about $x^{\prime}$ which is the axis of $\overrightarrow{B_{1}}$.

\section{Frequency Encoding}

To encode the MR signal in one dimension, one can frequency encode which means that during the readout a magnetic gradient field e.g. $G_{x}(t)$ is applied. As mentioned before, the Larmor frequency varies along the gradient direction and one line in $k$-space can be sampled with different frequencies. If spin-spin relaxation processes are neglected and without loss of generality a gradient $G_{x}(t)$ in $x$-direction is assumed, the signal equation (2.45) can be expressed as

$$
S(t)=\int_{x} M(x) \exp \left(-i \omega_{0} t\right) \exp \left(-i \gamma \int_{0}^{\tau} G_{x}(t) d t \cdot x\right) \mathrm{d} x \mathrm{~d} y \mathrm{~d} z
$$

with

$$
M(x)=\int_{z} \int_{y} M_{0}(x, y, z) \mathrm{d} y \mathrm{~d} z .
$$

Introducing $k_{x}(t)=\frac{\gamma}{2 \pi} \int_{0}^{\tau} G_{x}(t) \mathrm{d} t$ and demodulating the rotating frame, the measured signal can be identified as the Fourier transform of $M(x)$

$$
S\left(k_{x}(t)\right)=\int_{x} M(x) \exp \left(-i 2 \pi k_{x} x\right) \mathrm{d} x=F T(M(x)) .
$$




\section{Phase Encoding}

Spatial encoding in the third dimension takes place with a phase encoding gradient in $y$ direction. The so-called phase-encoder is a short magnetic field gradient which is applied between excitation and readout. While the gradient is switched on, spins precess with different frequencies according to the Larmor frequency of the effective magnetic field. Directly after switching off the gradient, the spins will precess with the system Larmor frequency again but with a different phase according to their position. Assuming the same as in section 2.3.1.2.4 and changing the gradient direction to the $y$-direction, it results in an equivalent equation to $(2.54)$

$$
S\left(k_{y}(t)\right)=\int_{y} M(y) \exp \left(-i 2 \pi k_{y} y\right) d y=F T(M(y)) .
$$

The equations $(2.54,2.55)$ describe the same effect as in chapter 2.3.1.2.4 with the difference that in case of phase encoding, the phase is modulated before the readout of the signal starts and in case of frequency encoding, the phase is modulated during signal acquisition.

\subsubsection{Influence of Sequence Parameters on Image Quality}

Apart from image artefacts (see section 2.3.5) the SNR is a significant descriptor of image quality. SNR can be defined, for example, as the average value of the signal in a homogeneous region divided by the standard deviation of the noise in a signal-free region. Typical factors which affect the SNR are the hardware used for imaging (MRI scanner) and all the details of the MR sequence. The nature of the sample (how lossy/noisy it is) is also very important but more difficult to influence than the imaging factors, especially so for in vivo imaging. The influence of some general sequence parameters on the SNR is discussed below.

1. Repetition time, TR: The repetition time is the time between two RF excitations. This time has a direct influence on the total acquisition time and should be as short as possible. However, a short TR does not allow for full $T_{1}$ relaxation between pulses, which results in a decreased MR signal in the next excitation. Thus, in general, SNR decreases as TR decreases.

2. The MR image is characterised, among others, by its matrix size and by the spatial extent of the field-of-view (FOV). The size of the voxels (FOV/matrix size in each direction) defines the spatial resolution of the image. Decreasing the voxel size (which corresponds to an increased spatial resolution) decreases the number of spins and total magnetisation in one voxel. Thus, all other things being equal, the SNR decreases with increasing spatial resolution. 
3. The number of independent samples of the noise influences the SNR by affecting the standard deviation of the noise: SNR $\approx \sqrt{\left(N_{x} N_{y}\right)}$. Furthermore, SNR will increase if the whole scan is repeated a number of times, $N_{\text {avg }}: \operatorname{SNR} \approx \sqrt{\left(N_{\text {avg }}\right)}$.

\subsubsection{Echoes: Spin Echoes and Gradient Echoes}

Following RF excitation the created transverse magnetisation $M_{x y}$ will decay with a relaxation time $T_{2}^{*}$. Depending on the desired contrast, it might be necessary to read out the signal after a long preparation time, when little signal is left because of $T_{2}^{*}$ decay. This is possible with the use of echoes. The so-called spin echo makes use of the possibility to reverse part of the $T_{2}$ dephasing - the $T_{2}^{*}$ part - of the spins. In order to achieve this, an additional RF pulse, a $180^{\circ}$ refocusing pulse, is inserted into the sequence at a time TE/2 after the first RF pulse excitation pulse. The $180^{\circ}$ pulse reverses the sign of the phase gained by the spins precessing in the xy-plane. Spins which were subjected to stronger dephasing and had accumulated a large phase now lag behind the spins subjected to no or moderate dephasing. The same field inhomogeneities as before act on the spins and all spins are rephased at the time TE after the excitation pulse. The MR signal at the time of the echo is much stronger than it would be in the absence of the $180^{\circ}$ pulse. Spin echoes compensate for the $T_{2}^{*}$ effects but not the $T_{2}$ relaxation. Therefore spin echo sequences are dedicated to generate $T_{2}$-weighted images.

The principle of the refocusing pulse is often compared to a $400 \mathrm{~m}$ race for illustrative purposes. We assume that every runner runs with a different but constant speed for a time $\mathrm{TE} / 2$, after which all athletes stop wherever they were on the track, reverse direction, and run with the same speed as before for another TE/2 interval. All will thus cover in the second TE/ 2 interval the same distance as in the first one and will arrive simultaneously at time TE at the start. At a time TE/2 when they first stopped they are said to be dephased; at time TE when they all reach the start line again, they are said to be rephased.

In contrast to the spin echo, the gradient echo does not compensate for $T_{2}^{*}$ decay, but provides a very convenient way to prepare the frequency-encoded signal directly for Fourier transformation. Initially, a gradient is used to pre-dephase all spins. With exactly the same but inverted gradient, it is possible to rephase all spins in the centre of the read-out. Due to this, all gradient echo sequences are $T_{2}^{*}$ weighted but they have the advantage of a much faster acquisition. A mathematical description can be found following. 


\subsubsection{Echoes}

In a rotating frame of reference, which is rotating at the centre frequency $\omega_{0}$, the effect on the phase of the signal which occurs because of gradient fields or off-resonance effects can be described with

$$
\phi(x, y, z, t)=\underbrace{\gamma E(x, y, z) t}_{\text {inhomogeneity }}+\underbrace{\omega_{c s t}}_{\text {chemical shift }}+\underbrace{\gamma \int_{0}^{\tau} G(t) \cdot r \mathrm{~d} t}_{\text {gradient fields }} .
$$

In MR almost every imaging sequence uses so-called echoes to acquire the signal. The echoes occur when the space-variant phase shifts are reversed. This can be achieved in two simple ways.

\section{Gradient Echo}

As mentioned before, a magnetic gradient field results in a modulation of the Larmor frequency and therewith in a dephasing of the spins. If the gradient direction is inverted, it results in a rephasing of the spin. Inverting the gradient direction means that the spins begin to rephase. After the absolute values of the amplitude-time-products of the rephaser and dephaser equals another, the spins are completely rephased and the echo occurs. The spins which are dephased by e.g. field inhomogeneities or chemical shifts cannot be rephased with a gradient echo and thus the signal decays faster as in the case of a spin echo. The relaxation constant which describes this behaviour is usually called $T_{2}^{*}$.

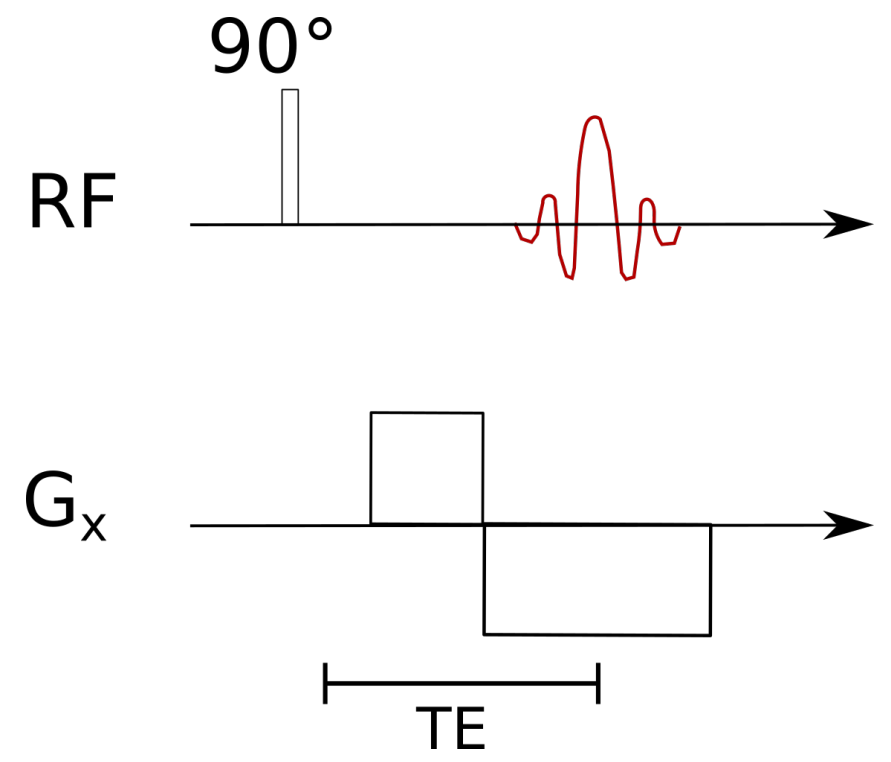

Figure 2.12: Schematic diagram of a simple gradient echo experiment with a $90^{\circ}$ excitation pulse and the corresponding echo displayed in red. 


\section{Spin Echo}

Another way to generate an echo is to use an refocusing pulse. If the $180^{\circ}$ refocusing pulse is applied after a time TE/2, the spins are dephased by means of chemical shifts and $B_{0}$ inhomogeneities during $0 \leq t \leq \mathrm{TE} / 2$ and rephased in the time $\mathrm{TE} / 2 \leq t \leq \mathrm{TE}$. This is caused by the same mechanism which lets the spins dephase. After the refocusing, the same effects cause rephasing of the spins. The exponential decay constant $T_{2}$ characterises the decrease of the amplitude at different echo times.

\section{$90^{\circ} 180^{\circ}$}
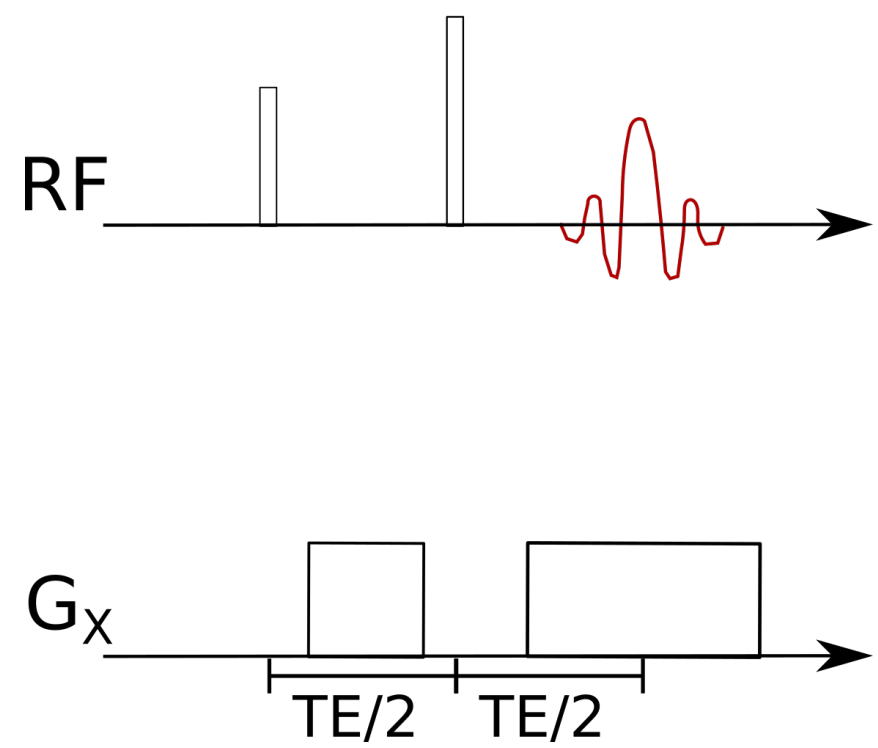

Figure 2.13: Schematic diagram of a simple spin echo experiment with a $90^{\circ}$ excitation pulse followed by a $180^{\circ}$ refocusing pulse and the corresponding echo displayed in red.

\subsubsection{Data acquisition in $2 \mathrm{D}$ and $3 \mathrm{D}$}

As already noted, the process of manipulating the spin ensemble is called a pulse sequence. Such a sequence usually comprises an excitation part with well-defined RF pulses, frequency and phase encoding, and an acquisition of echoes.

\subsubsection{2-dimensional}

Most commonly used imaging sequences sample in a 2D or 3D Cartesian manner. This means that the $k$-space of every single slice ( $z$-direction) is acquired on a Cartesian grid. Without loss of generality the readout direction is called $x$-direction. 
In the simplest case, one of $N$ lines ( $y$-direction) is acquired after a slice selective excitation with a gradient or spin echo. This results in $N$ excitations to sample one slice. These lines are selected by the use of a phase encoding gradient. The amplitude-time-product of the phase encoding gradient is varied for different lines.

\subsubsection{3-dimensional}

In contrast to a 2D sequence, excitation in 3D sequences can be nonselective. To achieve spatial information in $z$-direction, an additional phase encoding gradient is needed. This gradient varies the phase in $z$-direction and these steps are the so-called partitions.

The main advantages of a 3D acquisition in contrast to a 2D image are a higher SNR due to acquisition of the whole volume and not only a single slice and an easier achievable isotropic resolution and thiner "slices" in $z$-direction. In 2D acquisitions, the slice thickness is limited by the shape of the RF pulse and the gradient amplitude of the slice-select gradient. In 3D measurements, the thickness/resolution is determined via phase encoding. The major disadvantage of $3 \mathrm{D}$ acquisition is the long acqusition time caused by a long TR. In 2D sequences, the effective TR of a slice is given by TR - slices. If the same effective TR shall be used in 3D acquisitions, it has to be multiplied by the number of slices which prolongs the acquisition dramatically.

If a 3D Fourier Transform is applied to the 3D $k$-space, information is transfered to the object domain as mentioned in section 2.3.1.1.1.

\subsubsection{A simple imaging sequence}

Figure 2.14(a) depicts a typical 2D gradient echo imaging sequence and Figure 2.14(b) the responding $k$-space trajectory. Directly, after applying the slice selection gradient the spins are dephased and a slice rephasing gradient is applied. During this time, the phase encoding gradient is applied and the readout gradient is rephased.

The phase encoding gradient and the readout dephaser can be understood as a walk through the $k$-space as depicted in Figure 2.14(b). After applying these gradients the position is moved to, for example the upper left corner of the corresponding $k$-space. When the readout gradient is switched on, the analoge to digital converter (ADC), which samples the signal, is also switched on. The gradient echo occurs in the centre of the readout gradient because the readout dephaser has half of the area as the readout gradient. The time when the echo occurs is called the $T E$. This experiment is repeated $N$ times where $N$ is the number of phase encoding steps. 


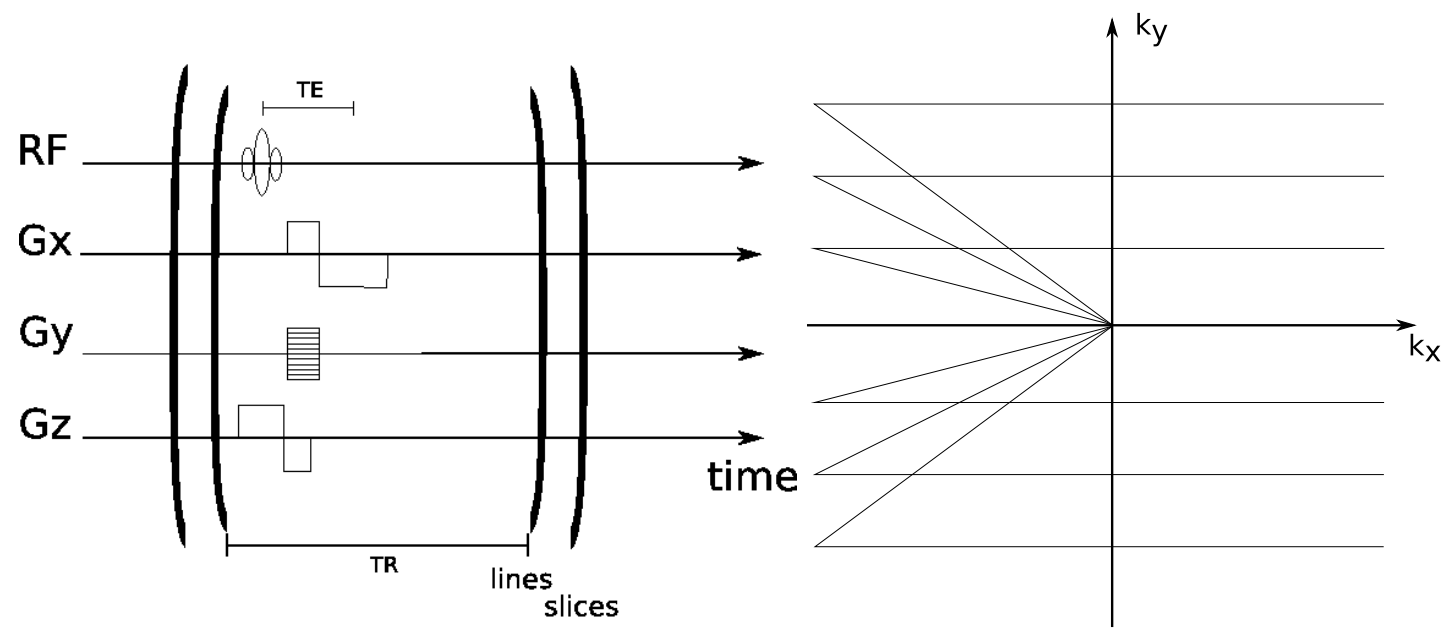

(a) Gradient echo sequence diagram.

(b) Typical $k$-space trajectory of a gradient echo sequence

Figure 2.14: Exemplary gradient echo sequence and the corresponding $k$-space trajectory for different repetitions of the phase encode gradient

\subsubsection{Artefacts}

MRI often suffers from image artefacts which are produced by different mechanisms and are sometimes unavoidable. The identification of these artefacts is important for the interpretation of MR images, especially for diagnostic purposes. In principle each sequence and combination of parameters can generate different artefacts and thus it should be addressed on a case-by-case basis and two frequent classes or artifacts are described below.

\subsubsection{Aliasing (Wrap-around)}

Since spatial information is encoded in the frequency domain, the MR signal should not contain frequencies higher than the highest frequency that can be digitised with the maximum ADC sampling rate. The Nyquist theorem, which is well known in signal processing, states that if a signal is sampled with sampling rate $1 / \Delta t$, the highest frequency that can be digitised accurately is $1 /(2 \Delta t)$. For the vast majority of MRI applications, the sampling rate of the ADC is chosen in such a way that all frequencies of the MR signal are well sampled. This argument, however, is only valid for the frequency encoding axis. The sampling rate along the phase-encoding axis is given by the increment of the phase encoding gradient. To understand what happens if the Nyquist criteria is not fulfilled, one has to realise that spatial encoding with the use of phase encoding only takes place in the chosen FOV. All spins outside the FOV are phase encoded in such a way that they cannot be distinguished 
from spins inside the FOV. This happens if the phase of the spins gains more than $360^{\circ}$ from the encoding. For example MR signals which underwent a phase encoding of $370^{\circ}$ behave like parts of the signal which are encoded with $10^{\circ}$. These parts are "wrapped around" to the other side of the image. This effect is called "wrap-around" or "fold-over". In signal processing it is generally called "aliasing". Choosing a larger FOV solves the aliasing problem, but at the expense of either measurement time or resolution. It is also possible to saturate parts of the imaged object in such a way that they do not contribute to the MR signal. The effective FOV can also be reduced by using special coils with spatially limited sensitivity.

\subsubsection{Chemical Shift}

The Larmor frequency of protons is influenced by the chemical environment, that is, the surrounding atoms, molecules, and their magnetic properties. The influence of the chemical environment on the frequency is called the "chemical shift". It is caused by a shielding of the static magnetic field (from the magnet) at the position of the hydrogen nucleus by the electron cloud and it is this shielding that is different in different chemical bonds (electron configurations). This property is extensively used in spectroscopy, to identify protons in different compounds by their chemical shift (see Figure 2.10). For imaging, however, the chemical shift can produce signal misregistration. A constant shift of frequencies is reflected by a spatial shift in images. This means that compounds containing protons with Larmor frequencies differing through chemical shift (e.g. water and fat are separated by $3.45 \mathrm{ppm}$ ) appear shifted relative to each other even if they are in the same spatial location. Some MRI sequences (such as EPI) are much more sensitive to these effects than others and the fat-water shift can be a significant proportion of the FOV. To minimise this effect it is customary to first suppress the signal of fatty tissue by use of narrow-band RF pulses which affect only the protons with the frequency corresponding to fat. The resulting fat signal can be dephased by the use of gradients. Subsequent imaging reflects the properties of water protons and no longer contains the contaminating signal from fat.

\subsubsection{3 $\mathrm{B}_{1}$ Inhomeogeneities}

The cause of spatial variation of the $\overrightarrow{B_{1}}$ excitation field is the coil which produces the $\overrightarrow{B_{1}}$ field and the finite wavelength which is given by

$$
\lambda=\frac{c}{\nu}
$$


where $c$ equals the vacuum speed of light and $\nu$ equals the frequency which is, in case of on-resonant excitation, well-defined by the Larmor frequency

$$
\nu=\frac{\gamma}{2 \pi} B_{0}
$$

which is in the case of proton imaging

$$
\frac{\nu_{\text {protons }}}{B_{0}}=42.56 \frac{\mathrm{MHz}}{\mathrm{T}} .
$$

The speed of light $c_{\text {med }}$ in a medium is influenced by the dielectric permittivity

$$
c_{\text {med }}=\frac{1}{\sqrt{\mu_{r} \mu_{o} \epsilon_{0} \epsilon_{r}}}
$$

where $\mu_{r} \approx 1$, if we assume only non-ferromagnetic materials. With increasing $B_{0}$ the Larmor frequency also increases. Therefore the wavelength for proton imaging decreases and approaches dimensions of the human body. Thus a varying $B_{1}$ distribution gives rise to differently measured signal intensities throughout the imaging area.

\subsubsection{Adiabatic pulses}

The aim of this section is to give a brief overview about the pros and cons of adiabatic pulses. For a more detailed description about adiabatic pulses the reader is referred to [28] and [29]. The main goal of adiabatic RF pulses avoid most of the $\overrightarrow{B_{1}}$ inhomogeneities such as mentioned in section 2.3.5.3. Standard RF pulses, such as a sinc or a rectangular pulse, are amplitude modulated and have a constant phase. Adiabatic pulses are amplitude and phase modulated and fulfil the so-called adiabatic condition [28] which is described in the following and is represented in equation (2.61).

\subsubsection{Basics}

In the early days of NMR, resonance was achieved using continuous wave (CW) excitation by sweeping the amplitude of the polarising magnetic field $B_{0}$ in the presence of a constant radio frequency field. This technique has been superseded by using pulsed NMR experiments. Here the static magnetic field $B_{0}$ is kept constant and a pulsed $\overrightarrow{B_{1}}$ RF field is used to excite the full band of spectral frequencies. Typically, the carrier frequency of these pulses is kept constant. So-called adiabatic pulses are frequency modulated pulses for 
which the carrier frequency is varied. In an adiabatic experiment, the net rotation of the magnetisation, $M$, is insensitive to amplitude modulations of the $\overrightarrow{B_{1}}$ field. These pulses offer the advantage of rotating the magnetisation by a constant flip angle, even if $\overrightarrow{B_{1}}$ is highly inhomogeneous. Within the spectral bandwidth of interest, all spins with different isochromats are rotated sequentially if the frequency sweep $\omega_{\mathrm{RF}}(t)$ approaches the resonant frequency of each isochromat. For adiabatic pulses such as the adiabatic full passage pulse - which is described below - the bandwidth is defined by the range of the frequency sweep. The flip angle which is applied to the spins is uniform if the effective magnetic field changes are slower than the rotation of $M$. This adiabatic condition can be satisfied by a slow frequency sweep or a high $\overrightarrow{B_{1}}$ amplitude.

For conventional pulses with a constant frequency, the bandwidth $\Delta \Omega$ is directly inverse to the pulse duration $T_{p}$. In many adiabatic pulses these two parameters are independent. The adiabatic condition $K\left(t_{\Omega}\right)$ can be expressed according to [28]

$$
K\left(t_{\Omega}\right)=\left|\frac{\left(\gamma B_{1}^{0} F_{1}\left(t_{\Omega}\right)\right)^{2}}{A \dot{F}_{2}\left(t_{\Omega}\right)}\right| \gg 1
$$

where $F_{1}$ represents an amplitude modulation and $F_{2}$ corresponds to a frequency modulation, $\Omega$ is the desired bandwidth and $t_{\Omega}$ represents all specific durations when the isochromat is on resonance. RF pulses which fulfil this adiabatic condition (2.61) can be used for excitation and for inversion.

The disadvantage of adiabatic pulses is a much higher energy deposition into the sample which causes problems in in vivo measurements because "specific absorption rate" (SAR) limits have to be accounted for. Another disadvantage of adiabatic pulses is a longer pulse duration to achieve the same flip angle compared to a standard pulse.

\section{Visualising Adiabatic Pulses}

Adiabatic pulses can be well understood in a second rotating frame of reference with axis labels $x^{\prime \prime}, y^{\prime \prime}, z^{\prime \prime}$ with respect to the usual used rotating frame with the axis labels $x^{\prime}, y^{\prime}, z^{\prime}$. This second frame of reference is the so-called " $B_{\text {eff }}$ frame" and it rotates with the frequency of the RF pulse. In standard RF pulses, the frequency is constant and therefore the angle between $B_{\text {eff }}$ and $M$ is large. Adiabatic pulses operate under the so-called adiabatic passage principle, which states that the magnetisation vector of a spin system follows the direction of $B_{\text {eff }}$, provided that the direction of $B_{\text {eff }}$ does not change significantly during one period of precession of the magnetisation about the effective field. Mathematically, this condition, also known as the adiabatic condition, is described by (2.61). When the adiabatic condition is fulfilled, a magnetisation which is initially collinear with $B_{\text {eff }}$ will remain collinear, and a magnetisation that is initially perpendicular to $B_{\text {eff }}$ will precess about $B_{\text {eff }}$ in a plane 
orthogonal to $B_{\text {eff }}$ during the pulse.

During an adiabatic full passage (AFP) pulse, which is described in more detail in section 2.3.6.2, the orientation of $B_{\text {eff }}$ is changed in such a way that the angle which is subtended by $M$ and $B_{\text {eff }}$ is kept constant throughout the pulse and the magnetisation $M$ moves with $B_{\text {eff. }}$ This implies the term "adiabatic", because the change in orientation of $B_{\text {eff }}$ has to be slow.
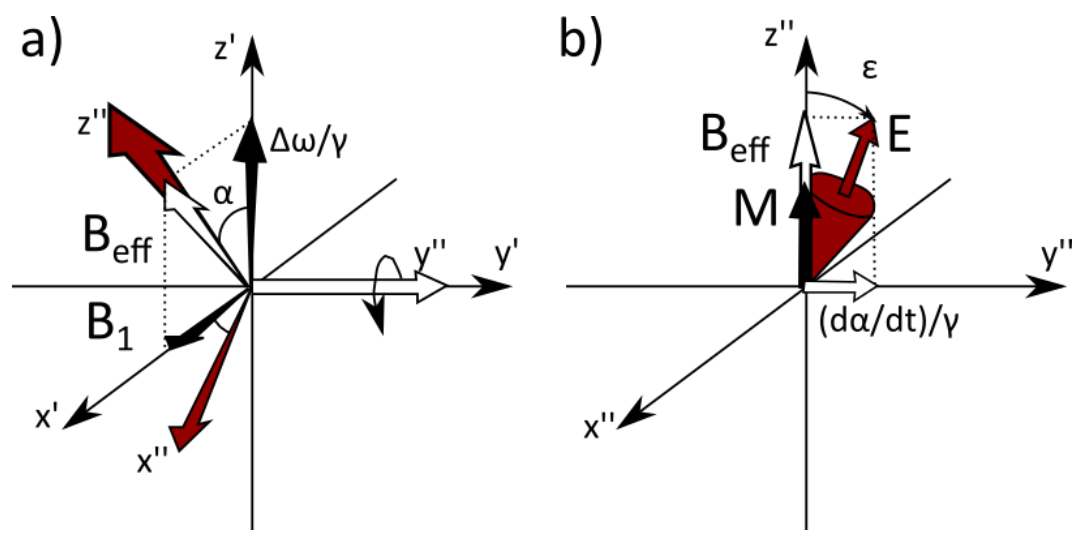

Figure 2.15: Vector diagrams showing the effective field and its components in two rotating frames of reference. In a) Relationship between the FM frame, $x^{\prime}, y^{\prime}, z^{\prime}$ with thin axes and the $B_{\text {eff }}$ frame, $x^{\prime \prime}, y^{\prime \prime}, z^{\prime \prime}$ with thick axes. In b) Magnetic field components and evolution of the magnetisation vector $\vec{M}$ in the $B_{\text {eff }}$ frame. Adapted from [28].

\subsubsection{Inversion Pulses}

One of the first adiabatic inversion pulses which produced a reasonably sharp profile is the hyperbolic secant or so-called adiabatic full passage pulse. The name of the pulse has its origin in the shape of the amplitude modulation which is given by

$$
\mathrm{amp}=\operatorname{sech}(\beta \cdot u)
$$

The phase modulation is given by

$$
\text { phase }=\mu \log (\mathrm{amp})
$$

where $\mu$ depicts the degree of phase modulation and $\beta$ the truncation level. Combining these gives

$$
\Delta \omega_{\mathrm{BW}}=2 \mu \beta
$$

which determines the bandwidth of the pulse. It is possible to solve the Bloch equations analytically with these amplitude and phase modulations.

The amplitude and phase modulation of this pulse with $-1 \leq u<1$ and $\beta=4.5$ and 
$\mu=5.0$ can be seen in Figure 2.16. The evolution of the magnetisation during a hyperbolic secant pulse is displayed in Figure 2.17 which was simulated using the open source MRI simulator JEMRIS $[30,31]$. As depicted in Figure 2.17, the longitudinal magnetisation is inverted relative slowly, to fulfil the adiabatic condition, while the transverse components oscillate during the pulse. After the pulse duration $T_{p}$, the longitudinal magnetisation is inverted and the transverse components are in the same condition such as before the pulse. This pulse results in a good inversion efficiency [32], is easy to implement and can be made

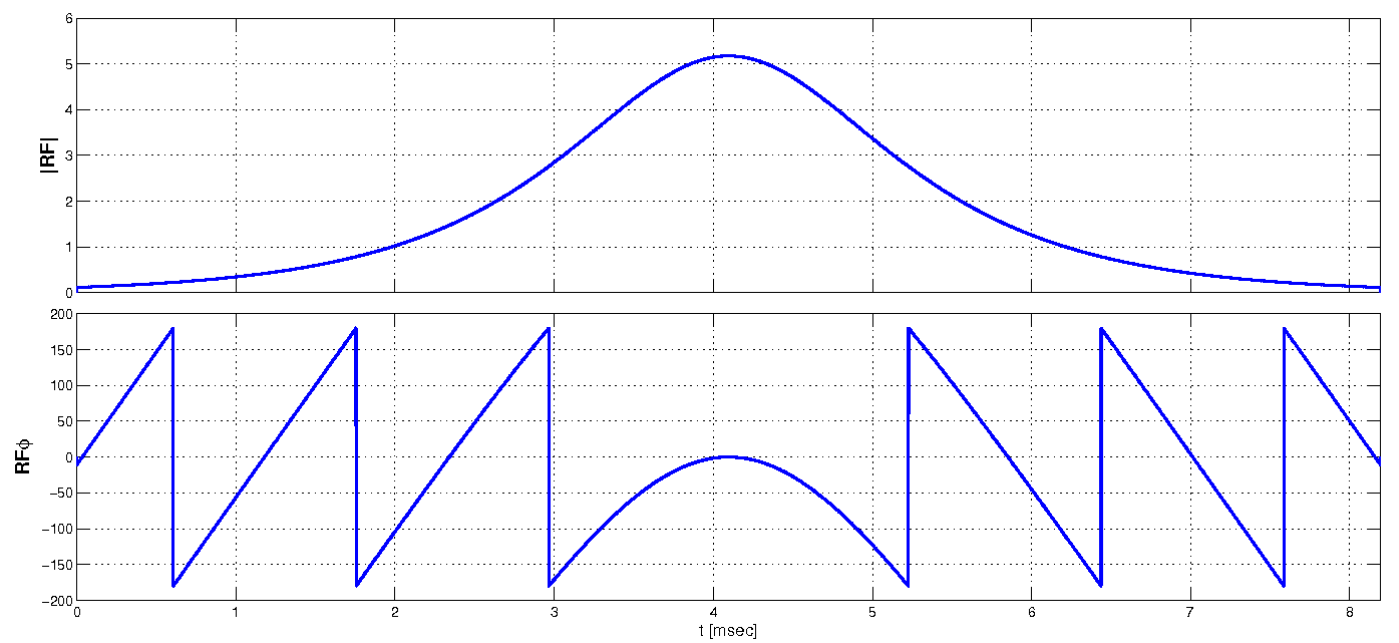

Figure 2.16: Amplitude and Phase modulation of the adiabatic full passage inversion pulse

slice selective. At the beginning of the pulse, the angle of $B_{\text {eff }}$ is 0 and thus, the effective field is aligned with the equilibrium magnetisation $M$ along the $z$-axis. If the adiabatic condition is satisfied the adiabatic passage principle dictates that the magnetization vector $M$ will track the direction of the effective field $B_{\text {eff }}$ during the RF pulse. More precisely $M$ precesses about $B_{\text {eff }}$ along a very tight cone. At half the pulse duration both $B_{\text {eff }}$ and $M$ point along the $x$ axis. During the next half of the pulse, $B_{\text {eff }}$ rotates toward the negative $z$ axis. At the end of the pulse, the effective magnetic field $B_{\text {eff }}$ has experienced a $180^{\circ}$ rotation. Since the

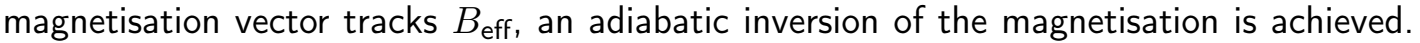
The trajectory of the magnetisation vector during adiabatic inversion is quite different from that in nonadiabatic inversion, in which case the magnetisation is always perpendicular to the $B_{1}$ field.

\subsubsection{Adiabatic RF Pulses with less peak power}

A relatively simple-to-implement adiabatic inversion pulse with less peak power and thus lower SAR deposition is the so-called HSn pulse [28]. These pulses allow an effective inversion and refocusing with less peak power during shorter pulse durations. Amplitude and 


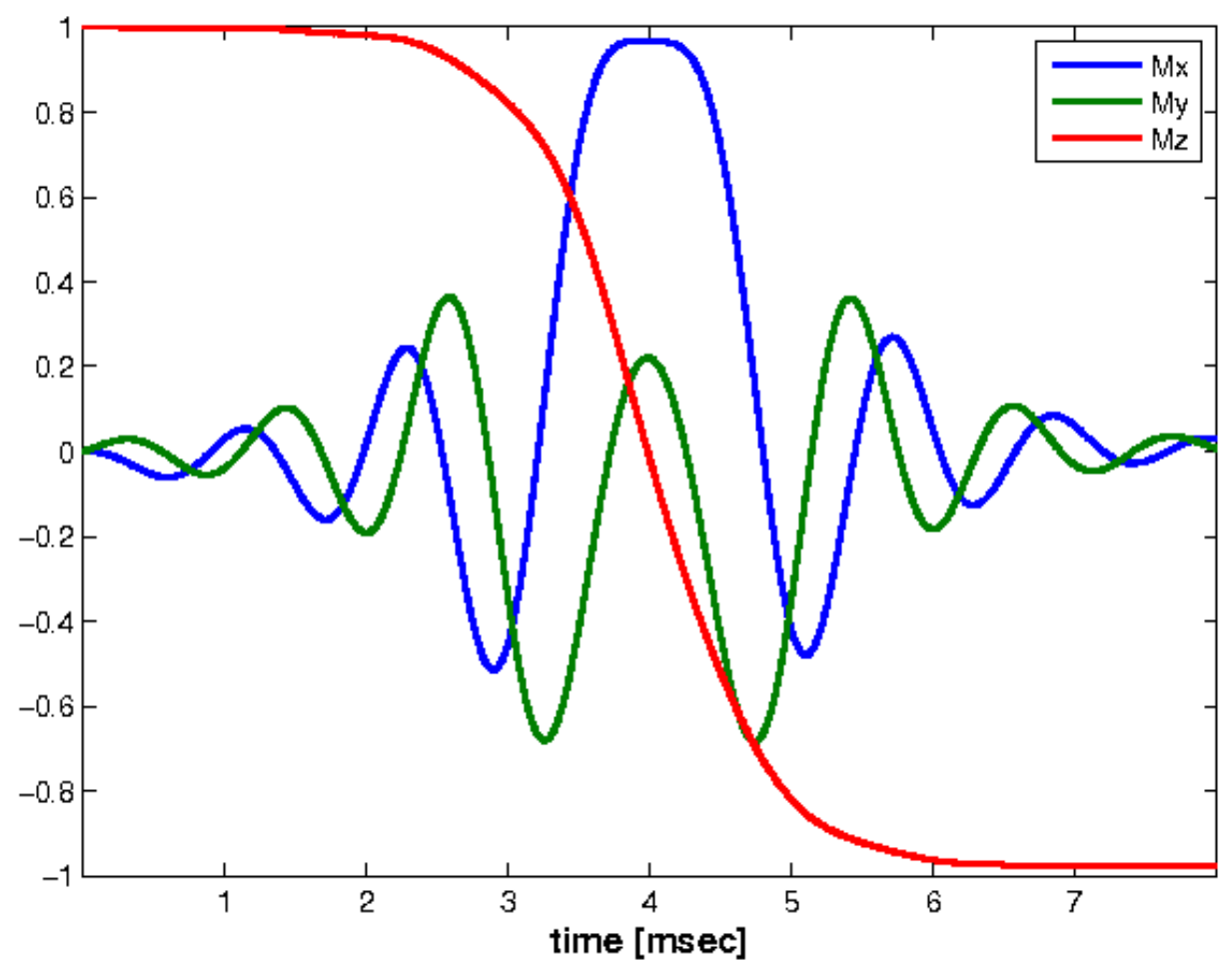

Figure 2.17: Evolution of the magnetisation during the AFP pulse

phase modulations of HSn pulses are shown in Figure 2.18 and given by $\left(\tau=\frac{2 t}{T_{p}}-1\right.$ for $\mathrm{t}$ in the range of $\left.\left[0, T_{p}\right]\right)$

$$
\begin{aligned}
\text { amp } & =\operatorname{sech}\left(\beta \tau^{n}\right) \\
\text { phase } & =\int \operatorname{sech}^{2}\left(\beta \tau^{n}\right) \mathrm{d} \tau
\end{aligned}
$$




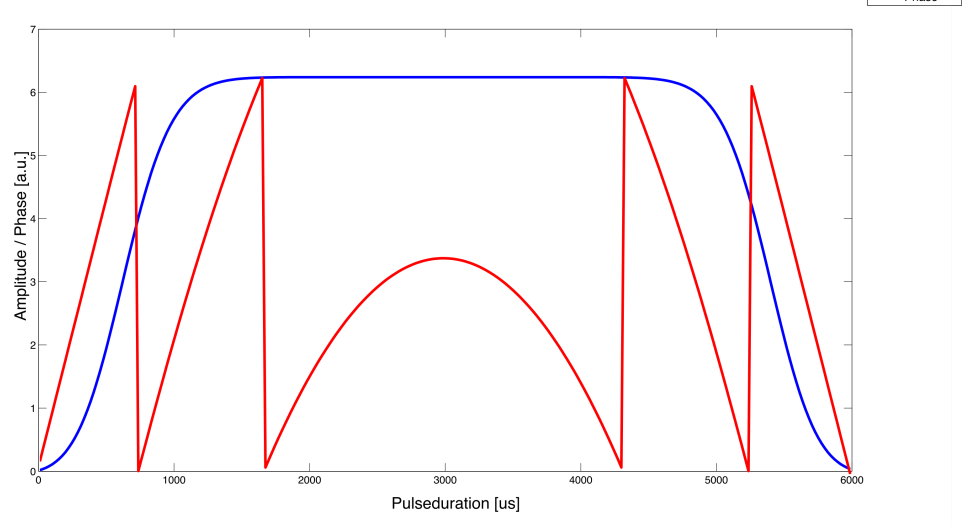

Figure 2.18: Amplitude and phase modulation of a HSn pulse with parameters: $n=6$; pulse duration $=6000 \mu \mathrm{s} ; \beta=5.3$; Bandwidth $=4000 \mathrm{~Hz}$. Phase is restricted to $[0,2 \pi]$ and amplitude is scaled to $[0,2 \pi]$ to achieve same size for better viewing.

\subsubsection{Parallel Imaging Techniques}

The main goal in parallel imaging is to speed up the whole imaging process. Classically, a coil with one element for receiving is used. For the use of parallel imaging more than one coil is needed. It is possible to acquire an image with each of the coil elements. To gain in acquisition speed, it is possible to acquire e.g. only every second line of the $k$-space and reconstruct the other ones with a GRAPPA (GeneRalized Autocalibrating Partially Parallel Acquisitions) [33] reconstruction.

Multicoil arrays [34] were designed to reach an increased signal-to-noise ratio (SNR) compared to volume coils. Another application of phased array coils is partially parallel acquisition (PPA) method, which is implemented to the image reconstruction system on the used 3 Tesla Siemens Tim-Trio system is the so called GRAPPA reconstruction method and described more precisely in section 2.3.7.2.

\subsubsection{History of Parallel Imaging Reconstruction}

Soon after the development of phased array coils [34], it was recognized that they could also be used to reduce scan time by utilising additional information given by multiple different coils and their own $B_{1}^{-}$field [35-38]. The basis of all such parallel imaging methods is that scan time is, as described in section 2.3.1.2, linearly proportional to the number of phaseencoding lines in a Cartesian acquisition. Increasing the distance between phase-encoding lines in $k$-space by a factor of $\mathrm{R}$, while keeping the maximal covered frequency in $k$-space 
fixed, reduces the scan time by the same factor. Increasing the distance between phaseencoding lines decreases the FOV by the same factor simultaneously. In parallel imaging, this factor $\mathrm{R}$ is called acceleration factor.

If the object extends outside the reduced FOV, aliasing or wrap-around artifact occurs (section 2.3.5.1). In PI techniques, the spatial dependence or sensitivity of the $B_{1}^{-}$field of the used receive coil array is used to reconstruct an unaliased image.

Two different approaches to remove the aliasing, one $k$-space based, for example GRAPPA [33], which itself is historically based on SMASH (simultaneous acquisition of spatial harmonics) [39] and one image space based, for example SENSE (sensitivity encoding) [40] exist.

In SMASH, the spatial dependence of the sensitivities is used to reconstruct synthetic missing $k$-space lines by approximating the corresponding sinusoidal phase twists produced by an encoding gradient from one single acquired line. Finally a single $k$-space data set is constructed and Fourier transformed to give the unaliased image. Further developed methods are AUTO-SMASH [41] in which a set of calibration lines are acquired around the centre of $k$-space and VD-AUTO-SMASH [42] in which these lines are used for latter image reconstruction as well.

SENSE [40] based image reconstruction is performed in the image space and not used during the work for this thesis and not further discussed.

\subsubsection{GRAPPA Reconstruction}

The prior described methods SMASH [39], AUTO-SMASH [41] and VD-AUTO-SMASH [42] were the first $k$-space based PI reconstruction methods but they are not used nowadays. GRAPPA [33] can be seen as a further developed VD-AUTO-SMASH method which applies, in contrast to all SMASH variants, a blockwise reconstructions to generate the missing lines and not only single lines. This is shown schematically in Figure 2.19. Data from multiple lines of all coils are fitted to an auto-calibration signal (ACS) line in a single coil. This results in some weights which are used to calculate all missing lines of the corresponding coil image. After applying this to all missing lines for each coil, a full set of uncombined images is obtained. These images can now be combined to one image using a normal sum of squares reconstruction.

The reconstruction of data from coil $j$ at a line offset $\left(k_{y}-m \Delta k_{y}\right)$ from the normal acquired data can be expressed by

$$
S_{j}\left(k_{y}-m \Delta k_{y}\right)=\sum_{l=1}^{L} \sum_{b=0}^{N_{b}-1} n(j, b, l, m) S_{l}\left(k_{y}-b A \Delta k_{y}\right) .
$$




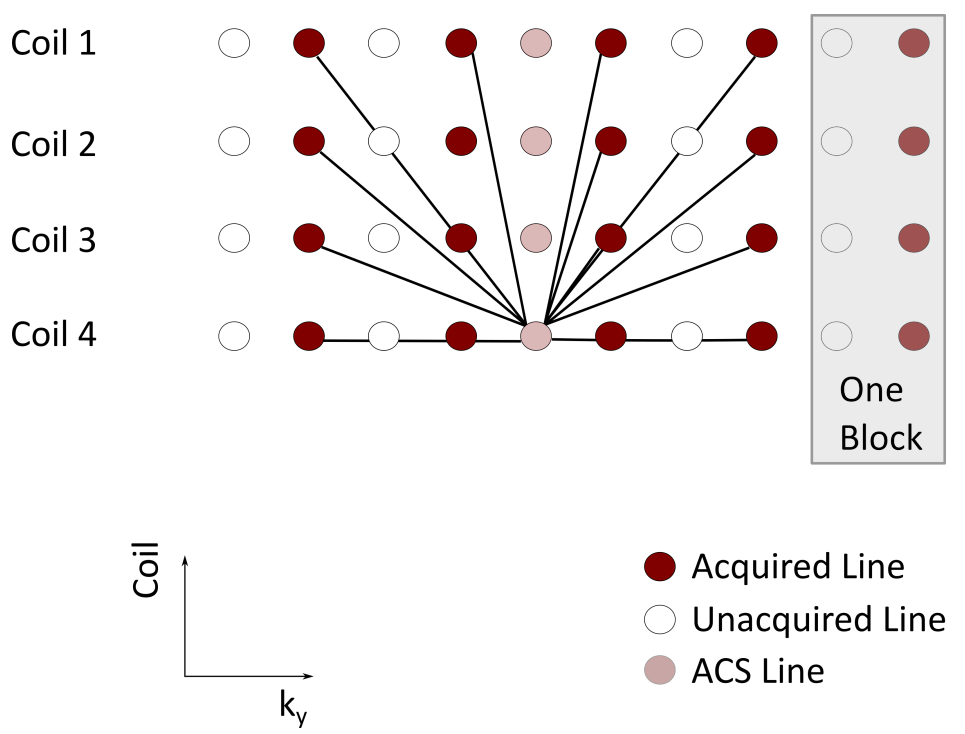

Figure 2.19: Schematic draft of the GRAPPA algorithm. Multi lines acquired in each of the coils are fit to an ACS line, which is acquired by a single coil. Here: 4 lines used to fit to a ACS line in Coil \#4. Adapted from [33]

For a more detailed description the reader is refered to [43].

\subsubsection{Multi Echoes and EPI Factor}

Echo planar imaging (EPI) is one of the fastest MRI pulse sequences [23] and with modern gradient and RF hardware, EPI is capable of acquiring a single 2D image in only a few tens of milliseconds. An EPI pulse sequence differs from conventional pulse sequences (such as spin echo and gradient echo as described in section 2.3.4.3), mainly in the ways that the readout and phase-encoding gradients are applied. In EPI a series of bipolar readout gradients is employed to generate an echo-train in with each gradient echo is distinctively spatially encoded via a phase encoding gradient pule. EPI is used to provide singleshot images with lower resolution. These images often suffer from artifacts such as ghosting along the phaseencode direction due to system imperfections (e.g. eddy currents) or chemical shift artifacts in the phase encoding direction (see section 2.3.5 for more details).

With so-called EPI-factors a multishot image acquisition is performed instead of a singleshot acquisition as in EPI and a standard gradient echo imaging sequence can be accelerated further by avoiding strong image artifacts as they appear in singleshot EPI images. With the use of multishot acquisitions, the effective acquisition bandwidth in phaseencode direction is increased and thus the appearance of chemical shifts artifacts are reduced.

If, e.g., three gradient echoes after one excitation are acquired and this is repeated $N / 3$ times, it is possible to speed up the acquisition process by a factor of three without having 
distortions.

This can be done in different ways. A first approach would be to acquire three consecutive lines. Thus the signal would be modulated periodically in the $k$-space, because of $T_{2}^{*}$ relaxation during the readout of the different lines. A simple alternative is to introduce interleaved segments, which are sampled after the excitation as depicted in Figure 2.20(a). The phase-encoding blip between the acquisition of the different lines has to have a greater momentum to reach the corresponding regions in $k$-space. This leads to a small time loss between the acquisitions in contrast to acquiring the lines consecutively but the aliasing artefacts - as they occur in convential EPI sequence - can be largely avoided [26]. The weighting caused by $T_{2}^{*}$ decay of the signal in $k$-space still leads to ringing in the image and thus the EPI-factor has to be well-chosen. Before image reconstruction takes place, the

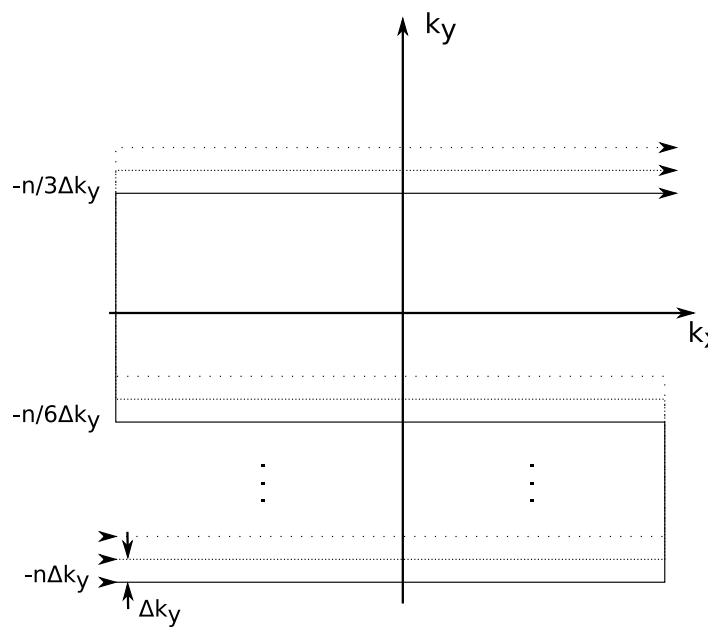

(a) $k$-space of a segmented EPI sequence

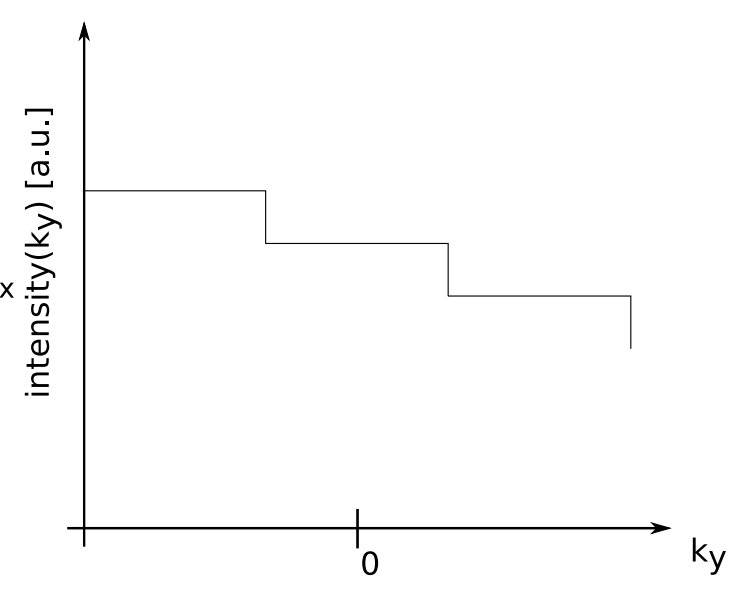

(b) relaxation weighting of the $k$-space

Figure 2.20: $k$-space diagram of a segmented EPI sequence and corresponding weighting of the intensity in $k$-space depending on the y-position.

lines, which were acquired with a negative gradient amplitude, have to be reversed because of time reversal, to be correctly registered to $k$-space.

Also phase correction of the acquired lines has to be performed to reduce ghosting artefacts [44]. These corrections are performed using the standard image reconstruction software, which is provided by the scanner. 


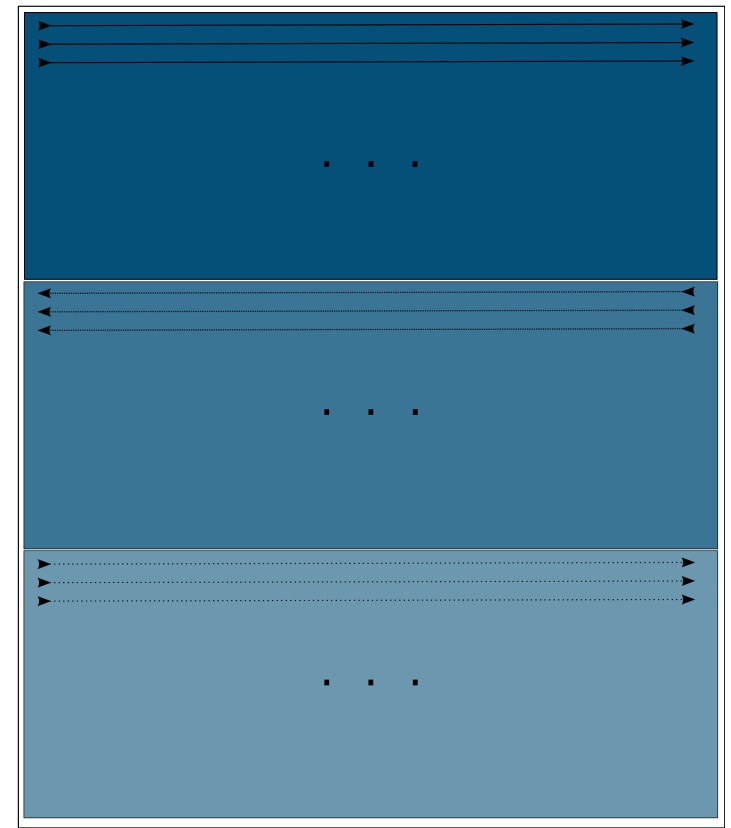

(a) segmented $k$-space with EPI factor 3

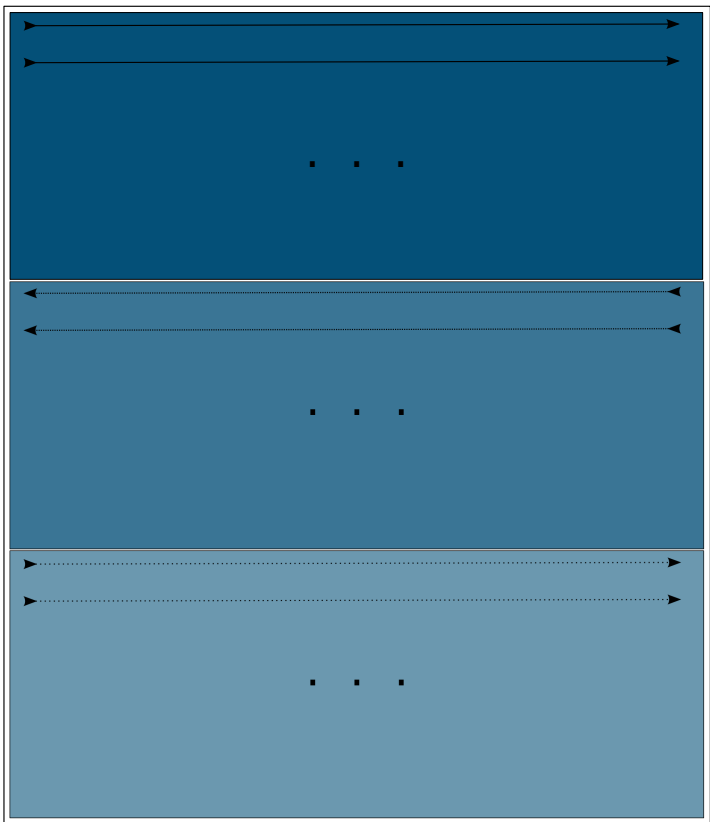

(b) segmented $k$-space with EPI factor 3 and GRAPPA 2

Figure 2.21: Depiction of a segmented $k$-space with EPI factor three.

By combining parallel imaging with the above mentioned segmented EPI readout scheme, the whole acquisition process can be accelerated by a large factor (e.g. combining EPI factor of 5 with an GRAPPA factor of 2 results in a total acceleration of approximately 10). This leads to faster imaging sequences which is a major advantage in the clinical acceptance of quantitative imaging.

\subsubsection{Echotimeshifting}

Multi-shot echo planar imaging, as it is used during the work for this thesis (see section 2.3.9 or section 3.3.2.2), suffers from field inhomoegneity related phase errors. Discontinuities in $k$-space result in ghosting artifacts in the image space as a result of the Fourier transform. As shown by Feinberg et. al $[45,46]$ the use of echo time shift (ETS) removes ghosting and modulation artifacts which are caused by recurrent identical phase errors in interleaved segmented or multi-shot EPI imaging sequences.

Off-resonance phase errors evolve continously with time during the gradient echo train and map directly onto the phase-axis of k-space. In single shot sequences there are no large discontinuous phase jumps but opposite polarity read gradients encode the consecutive signals so every other singal has to be reversed imposing a time reversal of the evolving 
phase error. In contrast multi-shot EPI different echo trains have identical off-resonance phase errors if they occur at the same time after excitation and thus signals with identical phase errors are grouped and phase discontinuity between these segments.

ETS makes use of a differential time delay $\Delta t$ between the refocusing pulse (in spin echo EPI) or the excitation pulse (in gradient echo EPI). Thus the echo train occurs at a different time on the phase error curve which is supposed to be constant. $\Delta t$ equals the spacing between next echoes in the train, $T_{G E}$ (total read gradient time including ramp up and down) divided by the number of excitation cycles $N_{E X}$. The time shift of the $i$ th excitation is given by

$$
\Delta t=(i-1)\left[\frac{T_{G E}}{N_{E X}}\right]-\frac{T_{G E}}{2}
$$

and visualised in Figure 2.22. As shown by Feinberg [46] this leads to less image artifacts due to a linear amplitude phase modulation instead of discontinuities. This only leads to negligible blurring in multi-shot EPI sequences and is implemented in all sequences used during the work for this thesis.
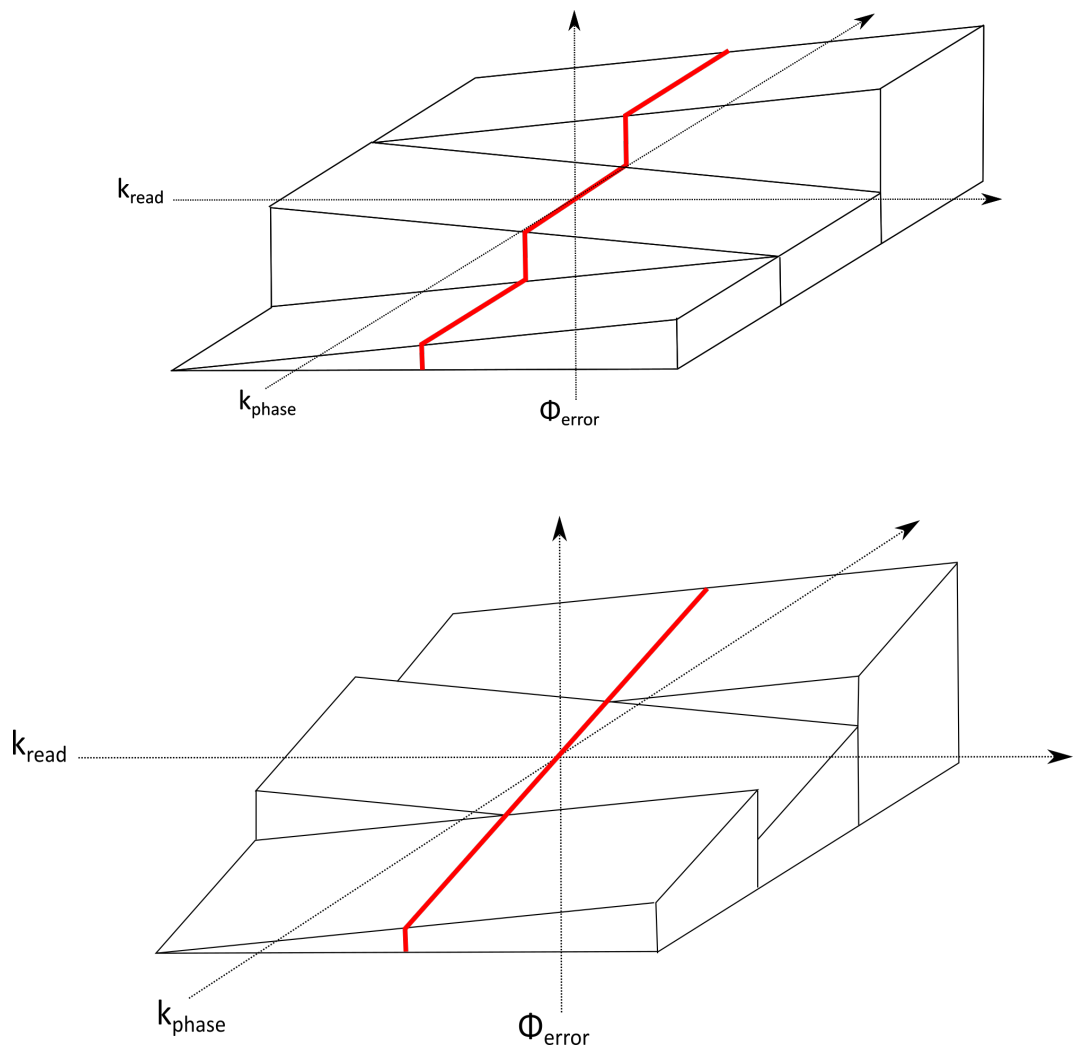

Figure 2.22: Evolution of the phase error in interleaved multi-shot EPI. Top: without ETS; bottom: with ETS. Here an echotrain of three echoes / segments is displayed. In spin echo EPI the phase error is zero in the middle of $k$-space, while for gradient echo EPI the phase error accumulates through the readout duration. It can be seen, that the phase evolution is a straight line (red) with the use of ETS while it has discontinuities if ETS is not used. Adapted from [46]. 


\subsubsection{Multi-shot multi-echo spin-echo EPI}

Nowadays single-shot EPI sequences are used for various purposes such as functional MRI and diffusion measurements, in which there is no need to achieve shortest possible echo times. As soon as quantitative $T_{2}$ imaging is performed, the need for short echo spacing and thus, in case of an EPI-like readout, the need for short readout times is obvious. In singleshot EPI sequences, this can only be achieved by the use of missing out parts in the k-space via parallel imaging techniques and partial Fourier skipping combined with low-resolution imaging. This shortens the readout time and thus the shortest possible TE and consequently echo-spacing which are beneficial to increase SNR. However, due to the inherent low SNR of the signal of interest averaging is still required increasing the measurement time. To overcome this drawback, we propose the use of a multi-shot segmented EPI readout. In this readout scheme, the $k$-space is divided in $\mathrm{n}$ segments (where $\mathrm{n}$ is the total number of acquired $k$-space lines divided by the number of shots). At each shot $\mathrm{n}$ lines (one line in each segment) is read out and this is repeated for all shots. Thus, the number of shots multiplies the total acquisition time, but the number of averages can be reduced due to higher available SNR consequence of shorter echo time and spacing.

\subsection{Safety Aspects}

MRI is a noninvasive medical imaging method, which does not use ionising radiation. At the current level of knowledge there is no evidence to suggest that MRI is associated with harmful effects; this aspect has been investigated since the first applications of NMR. There is, however, a need for safety-related precautions to be adhered to regarding all three main components of an MR scanner (main field, gradients, and the RF).

The static magnetic field interacts strongly with some types of materials. It has to be ensured that patients do not have any ferromagnetic implants or object on their person. The admittance of any ferromagnetic objects in the magnet room is strictly forbidden. These objects can be accelerated by the magnetic field to a velocity which can be compared to that of a projectile. Even small objects can have fatal effects when sharp. Obviously, although the main concern is for the safety of patient and healthcare staff. Accelerated ferromagnetic objects striking the magnet can also have serious effects on the magnet itself and cause it to change from being superconducting (no electrical resistance) to the normal, resistive state. When this happens, the magnetic field begins to decay suddenly and this event is known as a "quench". The sudden dissipation of heat causes the liquid helium to vaporise, creating a huge amount of helium gas at low temperature and very high pressure. If the gas is not channelled properly to the outside through a so-called quench pipe, the MR room 
will fill with gas very quickly after a quench, putting anybody in the room in danger of freezing and asphyxiation.

Another potential safety concern is direct electrical stimulation caused by the fast switching gradient fields. Electronic devices (e.g. pacemakers) can be easily damaged not just from the static field but also from the switching of gradients. Fast-switching gradients can induce currents into any conducting medium, for example the human body. This can result in direct electrical stimulation of peripheral nerves. Further effects include the generation of light flashes (magnetophosphenes) that may result from a slight torque exerted on the retinal cones. These biological side effects are circumvented by the imposition of limitations on the switching rate of the gradient fields $(d B / d t)$. The scanner operator is prevented from exceeding safety limits; the scanner software will simply stop the planned measurement from being executed until changes are made (switching times are lengthened, for example) such that the pertinent safety requirements are adhered to.

RF pulses can cause heating in the patient which is similar to the (much stronger) effect of microwaves and can cause heatings especially of ferromagnetic materials such as tattoos which contain ferromagnetic substances. Current FDA guidance limits the so-called SAR for whole body exposure to $4.0 \mathrm{~W} / \mathrm{kg}$ for patients with normal thermoregulatory function and $1.5 \mathrm{~W} / \mathrm{kg}$ for all patients, regardless of their condition. Again, the scanner operator is prevented from exceeding the safety limits by the software. 


\subsection{Oxygen Metabolism}

This section will describe some basic principles in the human body, first how oxygen from breathed air is transported in the blood in section 2.5.1 and finally how it is metabolised in the cells in the electron transport chain in section 2.5.2. The knowledge of the basic metabolisation processes is needed to understand and to develop new imaging technologies.

\subsubsection{Oxygen Transport}

Figure 2.23 shows the basic transport mechanisms in the human body. First air comprising oxygen is breathed in to the lungs. In the alveolis oxygen from the air is exchanged to the blood via osmosis and bound to haemoglobin in the erythrocytes. From there the blood is transported back to the heart and accelerated into the arterial system to the body. The arterial blood system ends up in capillaries where the bound oxygen is transported via osmosis to the tissue which can be e.g. muscles or the brain.

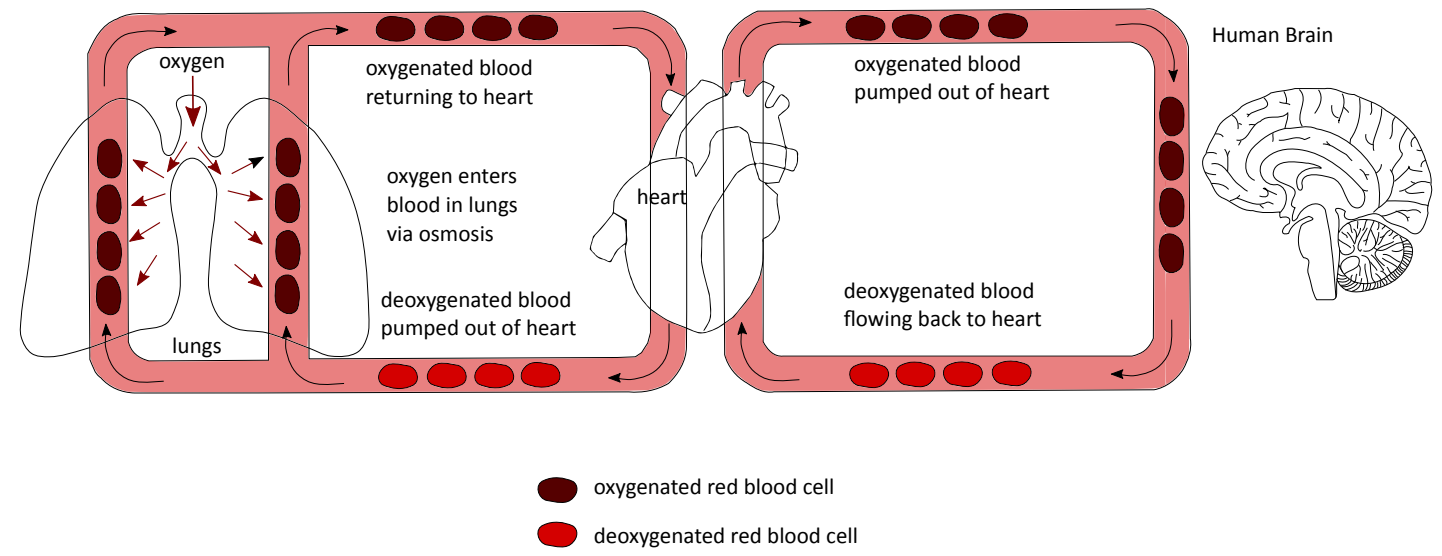

Figure 2.23: Schematic drawing of oxygen transportation and blood flow in the human body.

\subsubsection{Electron Transport Chain}

Following the transport of oxygen to the tissue, it is then metabolised from $\mathrm{O}_{2}$ to $\mathrm{H}_{2} \mathrm{O}$ in the electron transport chain (ETC). The ETC is a series of redox reactions which transfer electrons from electron donors to electron acceptors. This electron transfer is coupled with the transfer of protons across a membrane which creates an electrochemical proton gradient that drives the synthesis of adenosine triphosphate (ATP). The acceptor of electrons in the ETC is molecular oxygen and the final metabolisation step from oxygen to water happens in a redox reaction called Complex IV of the ETC, which is depicted in Figure 2.24. In Complex IV (or cytochrome $c$ oxidase) four electrons are moved from four molecules of cytochrome 
$c$ and then transferred to molecular oxygen $\left(\mathrm{O}_{2}\right)$. Which in turn produces two molecules of water. Simultaneously, four protons are removed from the mitochondrial matrix which contribute to the proton gradient. More details can be found in textbooks (e.g. $[47,48])$.

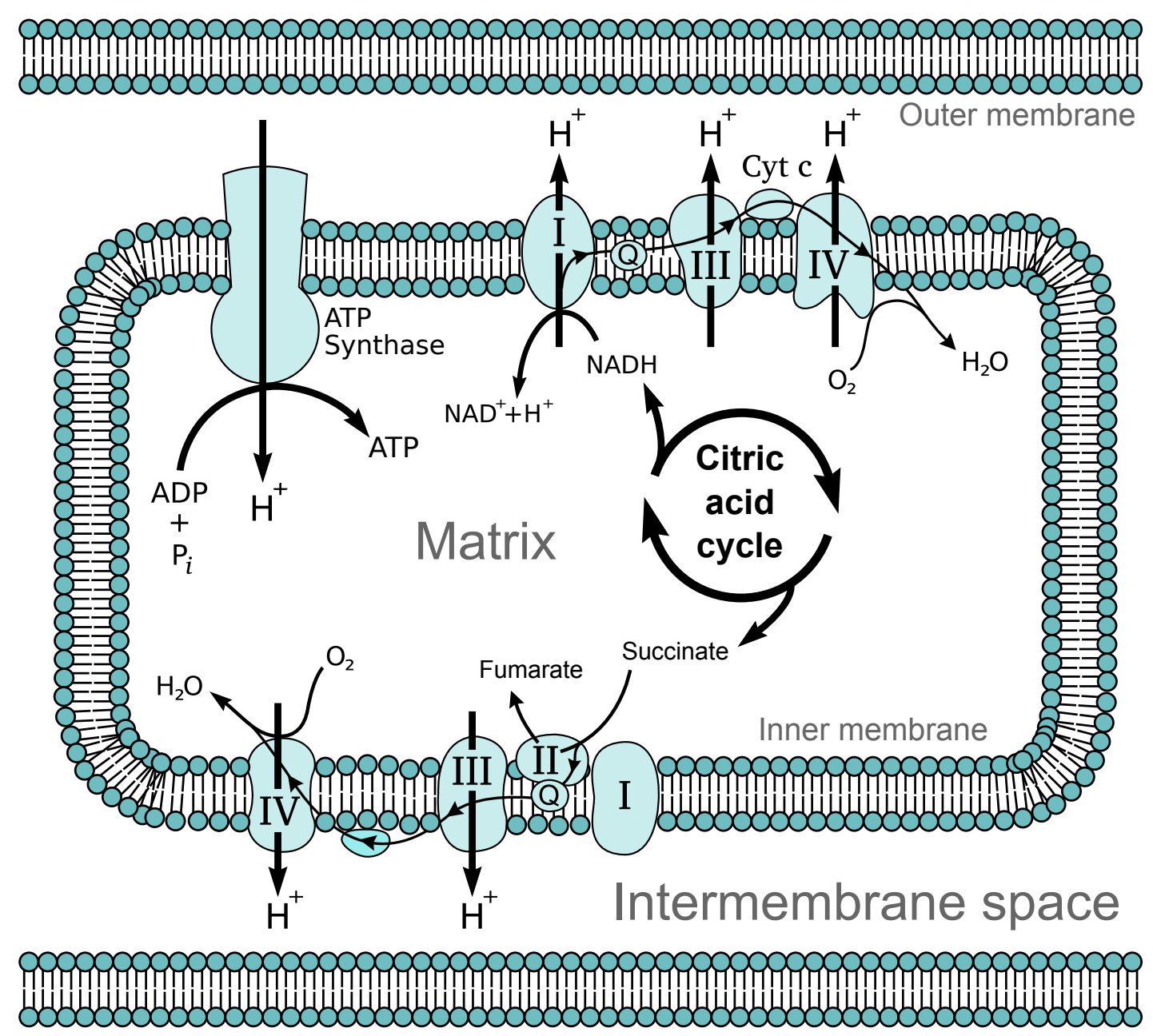

Figure 2.24: Schematic representation of the electron transport chain. At Complex IV $\mathrm{O}_{2}$ is metabolised to two $\mathrm{H}_{2} \mathrm{O}$. Figure taken from https://en.wikipedia.org/ wiki/Electron_transport_chain\#/media/File:Mitochondrial_electron_ transport_chain $\% 2 \% 80 \% 94 \mathrm{EtC} 4 . \mathrm{svg}$ which was originally published under the public domain. 


\subsection{Oxygen-17 $\left({ }^{17} \mathrm{O}\right)$}

Oxygen (molecular formula $\mathrm{O}_{2}$ ) is a colourless and odorless gas (at standard temperature and pressure which is according to IUPAC $T=273,15 \mathrm{~K}, \mathrm{p}=100 \mathrm{kPa}$ ). The two oxygen atoms are bond through a so-called double bond (spin-triplet electron configuration of an order of two).

Oxygen-16, which is the most abundand oxygen isotope, has eight neutrons an eight protons in its nucleus. Oxygen-17 ( $\left.{ }^{17} \mathrm{O}\right)$ was firstly hypothesized by Patrick Blackett [49] 1924 and imaged in Rutherford's lab 1924.

Blackett: "Of the nature of the integrated nucleus little can be said without further data. It must however have a mass 17 , and provided no other nuclear electrons are gained or lost in the process, an atomic number 8. It ought therefore to be an isotope of oxygen. If it is stable it should exist on the earth."

${ }^{17} \mathrm{O}$ is a stable isotope of oxygen with an additional neutron (with respect to ${ }^{16} \mathrm{O}$ ) in the nucleus and a nuclear magnetic moment $(\gamma=5.7 \mathrm{MHz} / \mathrm{T})$ and a half-integer spin of $\frac{5}{2}$ [50] which makes it suitable for NMR or MRI experiments [51-63].

In comparison to another isotope of oxygen, oxygen-15, which is used as an positron emission tomography tracer $\left({ }^{15} \mathrm{O}-\mathrm{PET}\right),{ }^{17} \mathrm{O}-\mathrm{MRI}$ can be used as a non-invasive method without any radioactive exposure due to $\mathrm{H}_{2}^{15} \mathrm{O}$ injection or inhalation of radioactive ${ }^{15} \mathrm{O}$. Another advantage of ${ }^{17} \mathrm{O}$ over ${ }^{15} \mathrm{O}$ is that no on-site production is needed. In case of ${ }^{15} \mathrm{O}$ experiments the tracer has to be produced on-site due to it's very short halflife of approximately 2 mins. [64]. Due to this benefits and the better availability of ultra-highfield MRI machines an increasing interest in ${ }^{17} \mathrm{O}$ MR spectroscopy (MRS) and MRI is seen during the last few decades.

A huge advantage of ${ }^{17} \mathrm{O}-\mathrm{MRI} / \mathrm{MRS}$ methods in contrast to ${ }^{15} \mathrm{O}-\mathrm{PET}$ is that ${ }^{17} \mathrm{O}_{2}$ molecules either in the form of free gas or dissolved in water are not MR-detectable due to the presence of two unpaired electrons making them strongly paramagnetic [58]. As an additional feature, when bound to haemoglobin, the ${ }^{17} \mathrm{O}$ signal is broadened so strongly that with the use of standard NMR techniques is not possible to measure the extremely slow rotational motion of the oxyhaemoglobin complex with its large molecular weight. This property causes very short transverse relaxation times $T_{2}$ leading the haemoglobin bound ${ }^{17} \mathrm{O}$ molecules being not detectable in NMR or MRI measurements.

Therefore, ${ }^{17} \mathrm{O}$ is only detectable by means of MR if it is bound to protons as water molecules $\left(\mathrm{H}_{2}^{17} \mathrm{O}\right)$ which is a unique feature of ${ }^{17} \mathrm{O}-\mathrm{NMR}$ techniques. This phenomenon allows the selective detection of metabolically generated $\mathrm{H}_{2}^{17} \mathrm{O}$ without confounding signals from the ${ }^{17} \mathrm{O}_{2}$ molecules bound to haemoglobin. This property is a huge advantage with respect to ${ }^{15} \mathrm{O}-\mathrm{PET}$ where the metabolically produced $\mathrm{H}_{2}^{15} \mathrm{O}$ and ${ }^{15} \mathrm{O}$ bound to haemoglobin are 
detectable thus impeding the differentiation between these two compartments.

Comparing the physical properties of ${ }^{17} \mathrm{O}$ to these of ${ }^{1} \mathrm{H}-\mathrm{MRI}$ the relatively small gyromagnetic ratio coupled to its scarce natural abundance $(0.037 \%)$, leads to a low NMR sensitivity $\left(2.9 \%\right.$ of $\left.{ }^{1} \mathrm{H}\right)$ complicating its detection and quantification [50].

The achievable SNR is strongly dependent on the available magnetic field strength as given by:

$$
\mathrm{SNR} \propto B_{0}^{\beta} \sqrt{\frac{Q T_{2}^{*}}{T_{1}}}
$$

where $\beta=7 / 4$, a theoretically predicted parameter, and $Q$ is the quality factor of the radiofrequency (RF) coil [65-67]. Thus ultra-high field (UHF) MR systems $(\geq 7 T)$ are usually the preferred choice in order to achieve an adequately high SNR.

In contrast to other nuclei (such as ${ }^{1} \mathrm{H},{ }^{31} \mathrm{P},{ }^{13} \mathrm{C}$ ), the relaxation times $T_{2}$ and $T_{2}^{*}$ and $T_{1}$ of ${ }^{17} \mathrm{O}$ are not largely determined by the dipole-dipole interactions. ${ }^{17} \mathrm{O}$ has a spin quantum number $I=\frac{5}{2}$ resulting in quadrupolar interactions and thus a shortening the relaxation times $\left(T_{2}^{*} \approx 2 \mathrm{~ms}, T_{1} \approx 4.8 \mathrm{~ms}\right.$ ) [50]. Subsidiary scalar coupling (between ${ }^{17} \mathrm{O}$ and ${ }^{1} \mathrm{H}$ ), which is depending on the proton chemical exchange rate of physiological solutions, leads to a further shortening of $T_{2}$ and $T_{2}^{*}$ [68].

It should be noted that quadrupolar interactions are field strength $\left(B_{0}\right)$ independent, so the fast $T_{1}$ and $T_{2}$ and $T_{2}^{*}$ relaxation times are not further influenced by the use of higher magnetic field strengths as it is for other nuclei as ${ }^{1} \mathrm{H}$. The field independence of the short ${ }^{17} \mathrm{O} T_{1}$ provides a useful advantage in terms of time efficient signal averaging at high fields [69] through averaging. Magnetic fields $\geq 7 \mathrm{~T}$, which are often used in research, generate a Boltzmann splitting which is large enough to achieve a sufficiently high SNR with ${ }^{17} \mathrm{O}$ at natural abundance. Due to the fast transverse relaxation rates standard Cartesian $k$-space sampling schemes are inefficient due to a relative long TE to perfom ${ }^{17} \mathrm{O}$ imaging which impedes the need for ultrashort echo time (UTE) sequences such as the twisted projection imaging (TPI) [70] are used and described in section 3.4.1. 


\section{Chapter 3}

\section{Methods}

In this chapter all methods employed in this thesis will be described. Initially, in section 3.1 , the different methods to quantify the cerebral metabolic rate of oxygen consumption $\left(\mathrm{CMRO}_{2}\right)$ based on either ${ }^{17} \mathrm{O}$ imaging, which is further described in section 3.2, and ${ }^{1} \mathrm{H}$ imaging, which is described in 3.1.4.

section 3.3 deals with the quantitative imaging methods, which are either the basis for the methods presented before or used as an additional input in the evaluation process.

\subsection{Quantification of $\mathrm{CMRO}_{2}$}

This section describes the most promising, currently available MRI-based methods for quantification of $\mathrm{CMRO}_{2}$. The first part in section 3.1.2 describes the methods and their mathematical concepts on how to quantify $\mathrm{CMRO}_{2}$ by using $\mathrm{x}$-nuclei imaging with ${ }^{17} \mathrm{O} \mathrm{MRI}$. The subsequent section 3.1.4 presents a method for clinical ${ }^{1} \mathrm{H}$ MRI and its further improvement.

\subsubsection{Cerebral metabolic rate of oxygen consumption}

Aging and, more importantly, healthy aging is becoming more important since the life expectancy is on the rise. At the same time many neurodegenerative and metabolic diseases associated with aging and pathologies such as Alzheimer's disease show altered values for $\mathrm{CMRO}_{2}$ [71-73] even before anatomical changes are detectable. Thus, a knowledge of quantitative values of $\mathrm{CMRO}_{2}$ is of great interest in order to follow and dynamically adapt patient treatment. Additionally, changes in $\mathrm{CMRO}_{2}$ are an active area in diabetes research where the central nervous system is assumed to play an integrative role.

Since decades, radioactive tracers based on ${ }^{15} \mathrm{O}$ were used to quantify $\mathrm{CMRO}_{2}$ via radioactive imaging modalities such as PET, with the latter being regarded as the gold standard [74]. 
As a consequence of the short half-life $(2 \mathrm{~min})$ of ${ }^{15} \mathrm{O}$, the methods are complicated and additionally expensive. Further, inherently they include radiation exposure which complicates studies on healthy volunteers due to its invasiveness.

Directly measured oxygen metabolism and a detailed knowledge of quantitative values for $\mathrm{CMRO}_{2}$ derived from NMR/MRI experiments may help to study altered brain metabolism in disorders such as the ones alluded to above and improves comparison with healthy control groups. One method to quantify $\mathrm{CMRO}_{2}$ is based on direct measurements of oxygen-17 $\left({ }^{17} \mathrm{O}\right)$. This method either needs ultrahigh-field MRI scanner $[61,75]$ or suffers from low resolution [76]. ${ }^{17} \mathrm{O}$ experiments are much easier to handle than ${ }^{15} \mathrm{O}$ PET imaging modalities, as there is no radiation exposure issues related to the short half-life ( $2 \mathrm{~min})$. However, ${ }^{17} \mathrm{O}$ enriched gas is expensive and a rebreathing circuit due to the high costs of enriched oxygen gas to limit the use of gas to less than 3 liters in e.g. 10 mins of breathing $[75,76]$ must be employed. Regardless of which method is used, the basic biological principles are the same and the oxygen extraction fraction (OEF) can be expressed via $\mathrm{CMRO}_{2}$ and $\mathrm{CBF}$ as follows $[77,78]$

$$
\mathrm{OEF}=\frac{\text { oxygen consumption }}{\text { oxygen delivery }}=\frac{\mathrm{CMRO}_{2}}{\left[\mathrm{HB}_{\mathrm{tot}}\right] \cdot \mathrm{CBF} \cdot Y_{a}}
$$

where $\left[\mathrm{HB}_{\text {tot }}\right]$ represents the total concentration of hemoglobin in blood and $Y_{a}$ represents the arterial and $Y_{v}$ the venous oxygen saturation. The OEF can be expressed according to [78] as a relationship between $Y_{a}$ and $Y_{v}$ via

$$
\mathrm{OEF}=\frac{Y_{a}-Y_{v}}{Y_{a}}
$$

\subsection{2 ${ }^{17} \mathrm{O} \mathrm{MRI}$}

Over the years a variety of methods were developed to quantify $\mathrm{CMRO}_{2}$ by measuring the ${ }^{17} \mathrm{O}$ signal which will be discussed in subsequent Sections. MRI based on a direct detection of ${ }^{17} \mathrm{O}$ enables $\mathrm{CMRO}_{2}$ quantification by comparison of the $\mathrm{H}_{2}^{17} \mathrm{O}$ signal increase to the natural abundance baseline [54-61].

It was shown that it is possible to calculate the CBF following injection of ${ }^{17} \mathrm{O}$-enriched physiological solutions [59] in spectroscopy (MRS) with additional measurements and by employing appropriate mathematical models [57-59]. ${ }^{17} \mathrm{O} \mathrm{MR}$ methods such as chemical shift imaging (CSI) provide the opportunity to determine the $\mathrm{CMRO}_{2}$ in different brain regions, by voxelwise comparison of the time course of spectral differences [79]. Imaging methods of ${ }^{17} \mathrm{O}$ provide voxelwise information as well and allow one to localise brain regions with different $\mathrm{CMRO}_{2}$ based on the imaging signal curve. Using imaging methods it has been demonstrated that white matter $\left(0.71 \pm 0.04 \frac{\mu \mathrm{mol}}{\mathrm{g} \mathrm{min}} \mu\right)$ has a lower $\mathrm{CMRO}_{2}$ than 
grey matter $\left(1.86 \pm 0.19 \frac{\mu \mathrm{mol}}{\mathrm{g} \text { min }} \mu\right)$ [61]. This method is non-invasive. Yet the development of the requiered mathematical models is a necessity, as different parameters such as the $\mathrm{H}_{2}^{17} \mathrm{O}$ contribution of perfusion from the rest of the body and the $\mathrm{H}_{2}^{17} \mathrm{O}$ loss factor, by exchange into the blood, have to be determined.

\subsubsection{Quantification of $\mathrm{CMRO}_{2}$ based on ${ }^{17} \mathrm{O}-\mathrm{MRI}$}

During the last few years, many groups have developed techniques which allow for a quantification of $\mathrm{CMRO}_{2}$ by means of spectroscopy [56-59]. Attempts to quantify $\mathrm{CMRO}_{2}$ via ${ }^{17} \mathrm{O}$ date back to the early 1990 s where enriched $\mathrm{H}_{2}^{17} \mathrm{O}$ was used as a contrast agent for ${ }^{1} \mathrm{H}-\mathrm{MRI}$ studies [80]. Thereafter, during the 1990s further efforts were made to calculate the CBF and $\mathrm{CMRO}_{2}$ by means of inhaling ${ }^{17} \mathrm{O}$ or by injecting solutions enriched with ${ }^{17} \mathrm{O}[58,59]$. However, all these efforts suffered from a low sensitivity and from the challenging properties of the ${ }^{17} \mathrm{O}$ nucleus. Additional difficulties came up in quantifying the parameters required to calculate the $\mathrm{CMRO}_{2}$. Thus, several mathematical models have been developed [57-60] in which the determination of additional parameters such as CBF is required.

One approach that has been developed by Zhu and Chen describes the $\mathrm{H}_{2}^{17} \mathrm{O}$ concentration changes in brain tissue during enriched ${ }^{17} \mathrm{O}$ gas inhalation $[57,58]$. The time-course of the signal of $\mathrm{H}_{2}^{17} \mathrm{O}$ is increased during inhalation of enriched ${ }^{17} \mathrm{O}$ gas [79]. Quantification of $\mathrm{CMRO}_{2}$ can be performed by assuming time-dependent $\mathrm{H}_{2}^{17} \mathrm{O}$ changes in excess of natural abundance within the brain tissue $C_{b}(t)$. This behaviour can be modelled via

$$
\frac{d C_{b}(t)}{d t}=2 \alpha f_{1} \mathrm{CMRO}_{2}+\mathrm{CBF}\left(f_{2}\left(C_{a}(t)-C_{v}(t)\right)\right)
$$

wherein $C_{a}(t)$ and $C_{v}(t)$ are the $\mathrm{H}_{2}^{17} \mathrm{O}$ fractions in the arterial and venous blood, $\alpha$ is the ${ }^{17} \mathrm{O}$ enrichment fraction of the inhaled ${ }^{17} \mathrm{O}$-air mixture and $f_{1}$ and $f_{2}$ are conversion factors $[57,58]$.

If further simplifications such as assuming fast water exchange rate in the capillaries in the brain are applied and if the CBF is estimated e.g. by a bolus injection of $\mathrm{H}_{2}^{17} \mathrm{O}$, one can modify (3.3) in such a way that $\mathrm{CMRO}_{2}$ can be quantified.

A mathematical model, which was evaluated by Atkinson and Thulborn [61], allows the quantification of metabolised water from dynamic experimental data on a voxelwise due to modeling the three phases (baseline / breathing room air; inhalation / breathing enriched oxygen; baseline / breathing room air) during the measurement. This model considers $\mathrm{H}_{2}^{17} \mathrm{O}$ signal changes during the inhalation period of enriched oxygen and requires three other parameters:

- $\mathrm{H}_{2}^{17} \mathrm{O}$ formation which represents the underlying $\mathrm{CMRO}_{2}$ 
- increase of the $\mathrm{H}_{2}^{17} \mathrm{O}$ due to diffusion/perfusion from the rest of the body $\left(K_{G}\right)$

- decrease of $\mathrm{H}_{2}^{17} \mathrm{O}$ due to loss by diffusion/perfusion into the blood, leading to chemical shift change due to metabolic conversion $\left(K_{L}\right)$.

All these factors are involved in the subsequently derived set of equations, expressing the signal changes of metabolised water, $\mathrm{M}_{V}^{\mathrm{H}_{2}^{17} \mathrm{O}}$, during the measurement time.

\subsubsection{1 $\mathrm{CMRO}_{2}$ quantification based on a 3-phase measurement}

As shown by Atkinson [61] the change of metabolised $\mathrm{H}_{2}^{17} \mathrm{O}, \mathrm{M}_{V}^{\mathrm{H}_{2}^{17} \mathrm{O}}$, can be calculated according to

$$
\frac{d M_{V}^{\mathrm{H}_{2}^{17} \mathrm{O}}(t)}{d t}=2 \cdot \mathrm{CMRO}_{2} A^{17} \mathrm{O}(t)-K_{L} \cdot M_{V}^{\mathrm{H}_{2}^{17} \mathrm{O}}(t)+K_{G} B^{\mathrm{H}_{2}^{17} \mathrm{O}}(t)
$$

wherein $A^{17} \mathrm{O}(t)$ is the concentration of arterial oxygen and $B^{17} \mathrm{O}(t)$ is the amount of $\mathrm{H}_{2}^{17} \mathrm{O}$ in excess of natural abundance. A depiction can be found in Figure 3.1. Apparently, this equation also contains unknown parameters $\left(K_{G}, K_{L}\right)$ which have to be determined in order to calculate $\mathrm{CMRO}_{2}$.

If the measurement is split into three phases as mentioned before, one can solve a set of

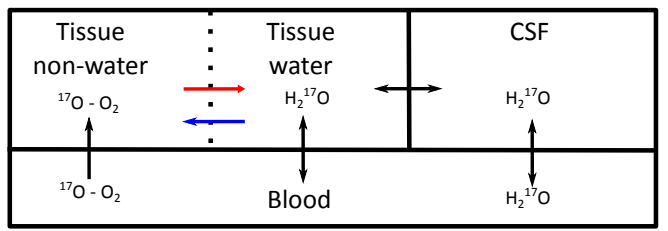

$\mathrm{H}_{2}{ }^{17} \mathrm{O}$ Osmosis / Diffusion $\longrightarrow$ 17-labeled $\mathrm{O}_{2}$ Diffusion $\longrightarrow \mathrm{H}_{2}{ }^{17} \mathrm{O}$ Generation $\longleftarrow \mathrm{H}_{2}{ }^{17} \mathrm{O}$ Conversion

(a) Multi-compartment model as used by Atkinson [61].

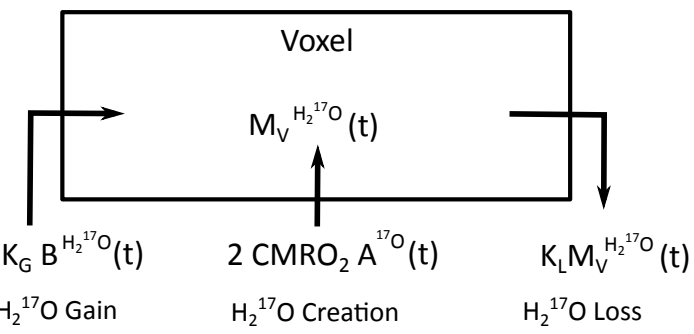

(b) Summary model of $\mathrm{H}_{2}^{17} \mathrm{O}$ generation and persistance. The total amount of the signal of $\mathrm{H}_{2}^{17} \mathrm{O}$ in one voxel depends on metabolically generated water, $\mathrm{H}_{2}^{17} \mathrm{O}$ gain due to diffusion from the blood and loss due to outward diffusion or chemical conversion.

Figure 3.1: Multi-compartment model as described by Atkinson and schematic representation of processes involved in signal changes within a single voxel. Adapted from [61]. 
equations. The derivation is described in detail in [61] and leads to the result:

$$
M_{V}^{H_{2}^{17} O}=\left\{\begin{array}{lr}
D & t<T_{A} \\
2 \cdot \alpha \cdot \mathrm{CMRO}_{2} \cdot\left(\frac{1}{K_{L}}-\frac{S_{T_{A}} e^{-\rho\left(t-T_{A}\right)}}{K_{L}-\rho}\right) \ldots & \\
+\frac{K_{G}}{K_{L}} \cdot \alpha \cdot\left(t-T_{A}+\frac{\left.S_{T_{A}} K_{L} \exp \left(-\rho\left(t-T_{A}\right)\right)\right)}{\rho\left(K_{L}-\rho\right)}-\frac{S_{T_{A}}}{\rho}-\frac{1}{K_{L}}\right) \ldots & \\
+c_{1} e^{-K_{L}\left(t-T_{A}\right)}+D & T_{A} \leq t \leq T_{B} \\
2 \cdot \alpha \cdot \mathrm{CMRO}_{2} \cdot \frac{1-S_{T_{A}} e^{-\rho T_{D}}}{K_{L}-\rho} e^{-\rho\left(t-T_{B}\right)} \ldots & \\
& +\frac{K_{G}}{K_{L}} \cdot \alpha \cdot\left(T_{D}+\frac{1-S_{T_{A}}}{\rho}\right) \ldots \\
-K_{G} \cdot \alpha \frac{1-S_{T_{A}} \cdot e^{-\rho T_{D}}}{\rho \cdot\left(K_{L}-\rho\right)} e^{-\rho\left(t-T_{B}\right)} \ldots & \\
+c_{2} e^{-K_{L}\left(t-T_{B}\right)}+D & t>T_{B}
\end{array}\right.
$$

where

$$
\begin{gathered}
c_{1}=2 \frac{\alpha \cdot \mathrm{CMRO}_{2}\left(\rho-K_{L}+K_{L} S_{T_{A}}\right)}{K_{L}\left(K_{L}-\rho\right)}-\frac{K_{G}}{K_{L}} \cdot \alpha \cdot\left(\frac{K_{L} S_{T_{A}}}{\rho \cdot\left(K_{L}-\rho\right)}-\frac{S_{T_{A}}}{\rho}-\frac{1}{K_{L}}\right), \\
c_{2}=c_{1} \cdot e^{-K_{L} T_{D}}-\frac{2 \mathrm{CMRO}_{2} \cdot \alpha \cdot \rho}{K_{L}\left(K_{L}-\rho\right)}+\frac{K_{G}}{K_{L}} \alpha\left(\frac{K_{L}}{\rho\left(K_{L}-\rho\right)}-\frac{1}{K_{L}}-\frac{1}{\rho}\right),
\end{gathered}
$$

$\rho$ is the rate with which the oxygen in the blood is exchanged, $\alpha$ is the level to which the fraction of ${ }^{17} \mathrm{O}$ labelled arterial oxygen gas in excess of natural abundance $A^{17} O$ is increased and $S_{T_{A}}$ is the oxygen saturation in the blood at time $T_{A}, T_{D}=T_{B}-T_{A}$ and $D$ the mole of $\mathrm{H}_{2}^{17} \mathrm{O}$ at natural abundance (equivalent to the water content in each voxel). Previous studies by Atkinson et al. [61] and Hoffmann et al. [75,81-83] use literature values of water content based on a segmentation in white and grey matter which could potentially fail in the presence of pathological changes. In the scope of this thesis, a method for quantifying the water content in the human brain based on ${ }^{1} \mathrm{H}$ MRI was developed and is described later in section 3.3.3.

By using this mathematical model, there is no need to calculate parameters such as the $\mathrm{CBF}$, which would require additional invasive measurements. The measurement procedure is based on the same experimental principle as ${ }^{17} \mathrm{O}$ spectroscopy by providing a breathing system that supplies a known quantity of ${ }^{17} \mathrm{O}$-enriched air $(70 \%)$.

\subsection{4 ${ }^{1} \mathrm{H}-\mathrm{MRI}$}

To quantify $\mathrm{CMRO}_{2}$ on the basis of ${ }^{17} \mathrm{O} \mathrm{MRI}$ experiments, one needs an extra device for recirculating the exhaled gas with enriched ${ }^{17} \mathrm{O}$. Such devices decrease patient comfort 
drastically which leads to a lower acceptance rate especially for patients with severe illness. To overcome issues like low resolution at clinical field strengths and to achieve a higher patient acceptance, ${ }^{1} \mathrm{H}$-based imaging modalities that do not require additional breathing masks during MRI are alternatives that hold potential. All implemented methods discussed here are based on the Fick principle of arteriovenous difference [1]. As shown in [84], $\mathrm{CMRO}_{2}$ can be written as

$$
\mathrm{CMRO}_{2}=\mathrm{CBF} \cdot\left(Y_{a}-Y_{v}\right) \cdot C_{a},
$$

wherein the CBF is the amount of blood flowing through the brain tissue in units of $\mathrm{ml} / 100 \mathrm{~g} / \mathrm{min} . Y_{a}$ and $Y_{v}$ represent the arterial and venous oxygenation (in \%) respectively. $C_{a}$ is a well-known constant in haematology [85] representing the amount of oxygen molecules, which can be carried by a unit of blood. If one considers that each gram of haemoglobin can carry $55.6 \mu \mathrm{mol}$ of oxygen [85] at a typical haematocrit (Hct) of 0.44 , the value of $C_{a}$ is $833.7 \mu \mathrm{mol} \mathrm{O}_{2} / 100 \mathrm{ml}$ blood. $C B F$ is measured by means of arterial spin labelling (ASL) using the same protocol in all measurements. In healthy subjects $Y_{a}$ can be assumed to be 1 . Alternatively it can easily be measured using pulse oximetry. The remaining variable $Y_{v}$ can be measured in different ways. The following methods are based on changes in the transverse relaxation rate of venous blood in proton MRI with different blood oxygenation levels. While the TRUST method is faster with respect to the total acquisition time, the QUIXOTIC method provides localised $\mathrm{CMRO}_{2}$ values instead of one single whole-brain $\mathrm{CMRO}_{2}$ value as acquired with the TRUST method.

\subsubsection{From venous blood $T_{2}$ to $Y_{v}$ values}

In the early 1980s, Thulborn et al [86] discovered a relationship between blood oxygen saturation and transverse relaxation times in MR. Later a parametric model, which in turn is based on the Luz-Meiboom model [87] of spin relaxation during two-compartment exchange, was derived. This enabled first MRI measurements of oxygen saturation in vivo [86] by estimating $T_{2}$,blood. $T_{2}$ relaxation rates of blood can be linked to physiologic parameters including the oxygenation $Y$ according $\operatorname{Van} \mathrm{Zijl}[77,88]$ via

$$
\frac{1}{T_{2, \text { blood }}}=A+\operatorname{Hct}\left[(B+C(1-Y))+(1-\mathrm{Hct})(D+E(1-Y))^{2}\right],
$$

where Hct represents the hematocrit of the blood and $A, B, C, D$ and $E$ represent lumped biophysical parameters of the blood related to susceptibility differences of red blood cell and plasma, water relaxation rates in red blood cells and plasma and the spacing between the refocusing pulses in a CPMG spin-echo module $\left(\tau_{\mathrm{CPMG}}\right)$. These values were estimated by Bolar [14] on the basis of data obtained at a clinical $3 \mathrm{~T}$ MRI from freshly extracted 
bovine blood with known Hct and $Y_{v}$ values to be:

$$
\begin{aligned}
& A=1.09 \frac{1}{s} \\
& B=11.26 \frac{1}{s} \\
& C=-7.96 \frac{1}{s} \\
& D=1.08 \frac{1}{\sqrt{s}} \\
& E=16.54 \frac{1}{\sqrt{s}} .
\end{aligned}
$$

These values are used in this work.

Using $Y_{v}$ one can calculate $\mathrm{CMRO}_{2}$ and OEF via (3.1) and (3.2) combining a hematocrit measurement to calculate the total hemoglobin concentration via

$$
\left[\mathrm{HB}_{\mathrm{tot}}\right]=\frac{\mathrm{Hct}}{3.0\left(\frac{\mathrm{ml}}{\mathrm{g}}\right) \cdot 0.016125\left(\frac{\mathrm{g}}{\mu \mathrm{mol}}\right)} .
$$

The missing quantitative value for arteriolar blood is either assumed to be fully saturated $\left(Y_{a}=1\right)$ or measured independently by a pulse oximetry.

\subsubsection{Multi Echo Spin Echo}

As mentioned in the previous section 3.1.4.1, $Y_{v}$ can be calculated via known transverse relaxation rates of venous blood. Since (see section 2.3.3) in a spin echo experiment the signal is weighted $T_{2}$ and not by $T_{2}^{*}$ as in a gradient echo experiment, the simplest method, to quantify $T_{2}$ in general is done with performing spin echo experiments at different TE. First, images are acquired at increasing TE. The signal intensity decays with respect to TE in an exponential way with the relaxation time $T_{2}$ and thus allows for a simple voxelwise exponential fit of the data. This results in a quantitative $T_{2}$ map of the corresponding image volume. The repetition time $T R$ has to be kept long ( $T R \approx 10 \mathrm{~s}$ or even longer) to achieve full relaxation, this method is relatively time consuming. One approach to acquire all relevant images at all different TE in one go, is to use a multi-echo, spin-echo sequence. In quantification procedures of $T_{2}^{*}$ a multi-echo, gradient-echo sequence, such as QUTE [89-91], is widely used. If this approach is adapted to spin echo acquisitions basic MR principles have to be taken into account to avoid stimulated echo generation. A basic multi-echo, spin-echo sequence is depicted in Figure 3.2. The FID signal decays with $T_{2}^{*}$ and the signal intensity of each spin echo decays with $T_{2}$. 


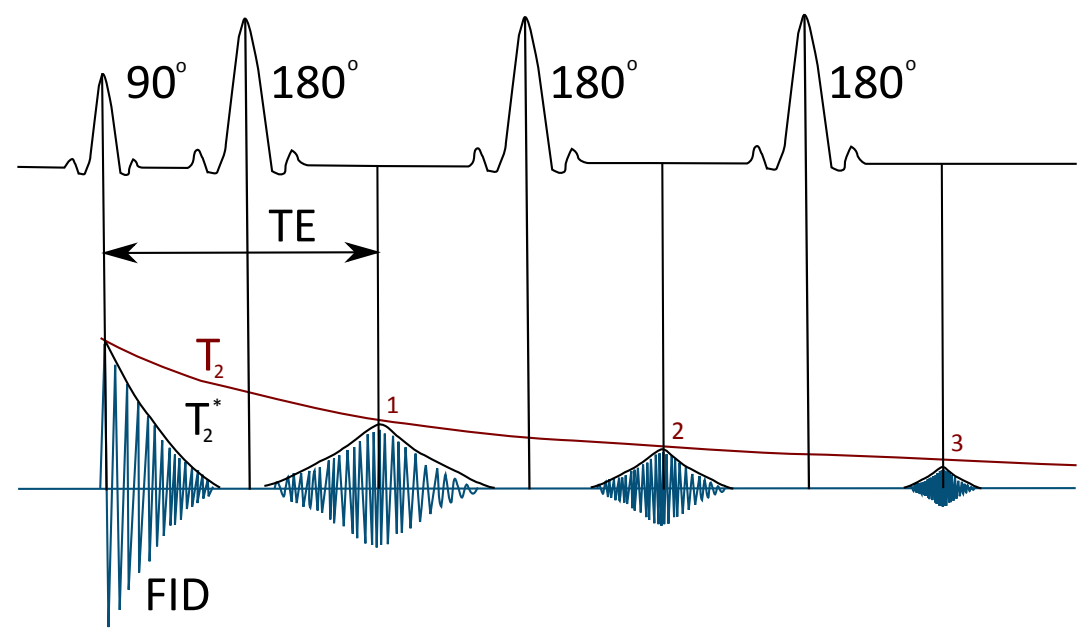

Figure 3.2: Schematic representation of a multi-echo, spin-echo train including depiction of $T_{2}$ and $T_{2}^{*}$ signal decay.

\subsection{Stimulated Echoes}

Quantitative imaging and quantification of $T_{2}$ is being investigated since decades. It is wellknown that simple multi-echo, spin-echo approaches are prone to errors due to RF pulse imperfections that generate stimulated echoes [92-95]. Since imperfect $180^{\circ} \mathrm{RF}$ pulses (due to non-ideal pulses or $B_{1}^{+}$inhomogeneities) do not refocus the transverse magnetisation in the selected volume completely, parts of the initially generated transverse magnetisation are tipped back into the longitudinal plane and are thus subject to $T_{1}$ relaxation processes. Subsequent $180^{\circ} \mathrm{RF}$ pulses generate transverse magnetisation and refocusses it again which gives rise to unwanted echoes with mixed relaxation time dependencies. One approach to overcome the problem of refocusing this magnetisation and thus avoiding stimulated echoes is based on variable gradient spoiling and will be described in the following section . Another approach to avoid tipping the transverse magnetisation back to longitudinal is the use of adiabatic refocusing pulses (see section 2.3.6), which are much less prone to $B_{1}^{+}$ inhomogeneities.

\section{Spoiling Scheme}

Stimulated echoes caused by hard rectangular RF pulses become much more prominent than if adiabatic refocusing pulses are used. To overcome this issue a gradient spoiling scheme is introduced. In normal CPMG SE sequences a pair of gradients are used on the read and slice axis before and after the refocusing pulse to dephase transverse magnetisation. This leads to stimulated echoes right after the third RF pulse (at TE of the second $\mathrm{SE}$ ) and thus $T_{2}$ quantification is no longer accurate. Poon and Henkelman introduced an efficient gradient spoiling scheme in 1992 to avoid stimulated echo contribution [93] which 
is implemented in this sequence. as schematically represented in Figure 3.3.

\begin{tabular}{c|c} 
Pulse Number & Relative Momentum \\
\hline 1 & $N / 2$ \\
2 & $-(N / 2)$ \\
3 & $N / 2-1$ \\
4 & $-(N / 2-1)$ \\
5 & $N / 2-2$ \\
6 & $-(N / 2-2)$ \\
$\vdots$ & $\vdots$ \\
$N-1$ & 1 \\
$N$ & -1
\end{tabular}

Table 3.1: Gradient spoiling scheme for the use in multi-echo, spin-echo sequences as suggested by Poon et al. [93]. Data is expressed as the relative gradient strength at each pulse. $N$ is assumed to be even.

In multi-echo, spin-echo sequences the use of refocusing pulses generates stimulated echoes which contain additional $T_{1}$ relaxation information and thus generate an unwanted signal contribution. One way to overcome this issue is to use a spoiler pattern on the slice selection gradient axis which dephases all magnetisation in such a way that it will not rephase in later echoes. For $N$ echoes this can be sufficiently achieved with a spoiling pattern as shown in Table 3.1.

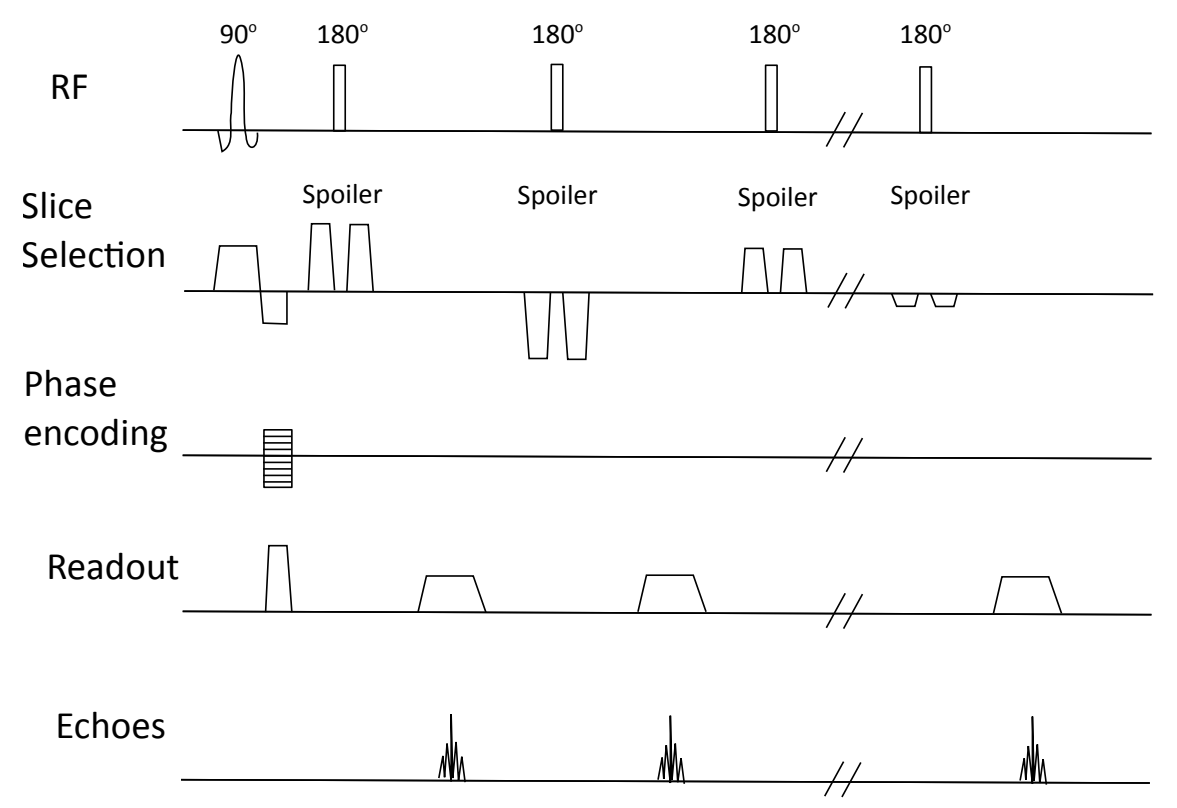

Figure 3.3: Imaging sequence diagram with gradient spoiling scheme as suggested by Poon et al. [93]. 
A simulation of the magnetization in both spoiling schemes according to the phase-graph formalism, which is described in detail in [96], can be found in Figure 3.4. The top part of the graph shows the gradient spoiling on the z-axis and the lower part its effect on the various states generated by RF-pulses (shown with dotted vertical lines).

Figure 3.4a) presents the case of equal spoiling momentums as is it used in CPMG sequences. Figure $3.4 \mathrm{~b}$ ) presents the diversification of the different states if the spoiling scheme introduced by Poon and Henkelman [93] is used. It is clearly visible that the use of equal spoiling lead all states which form to stimulated echoes coincidence with the spin echo pathways and thus generate an unwanted signal contribution. Figure 3.4b) shows that it is not the case if the alternating spoiling scheme is used and all pathways are separated and pure spin echoes are formed. 

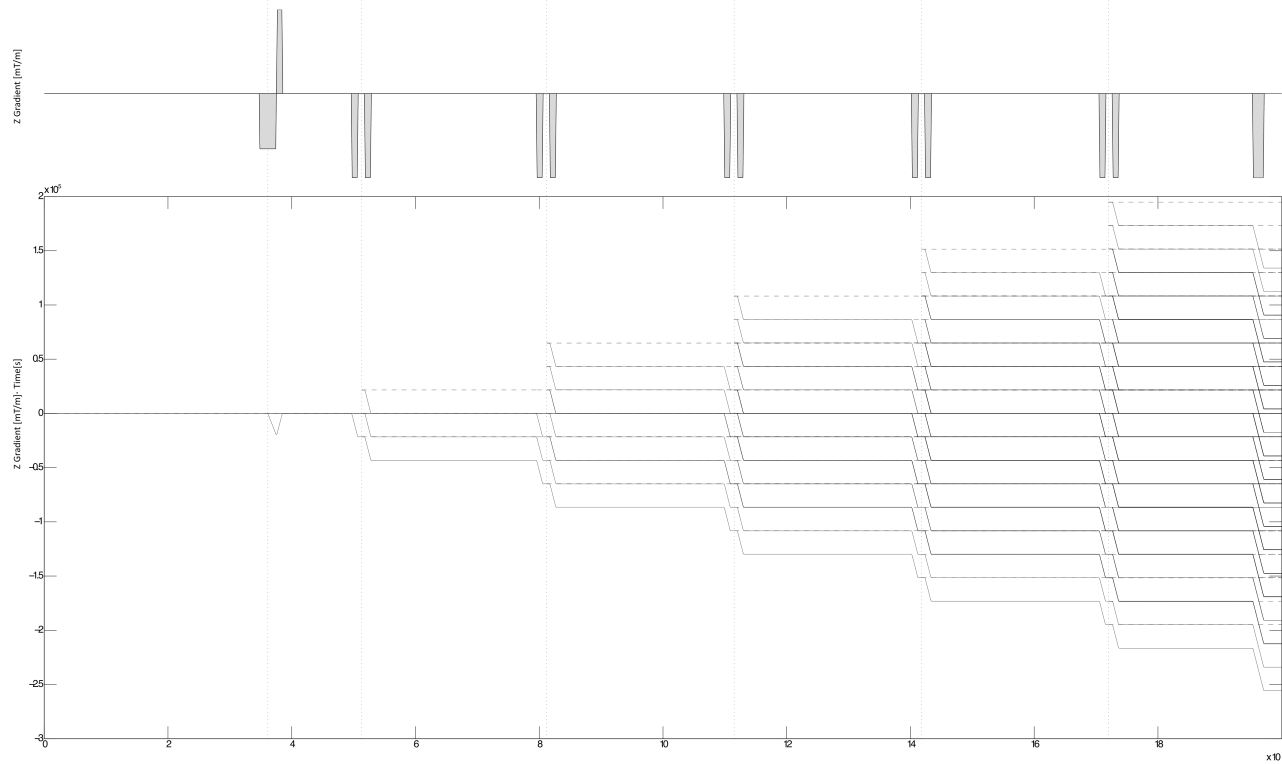

(a) Equal spoiling scheme

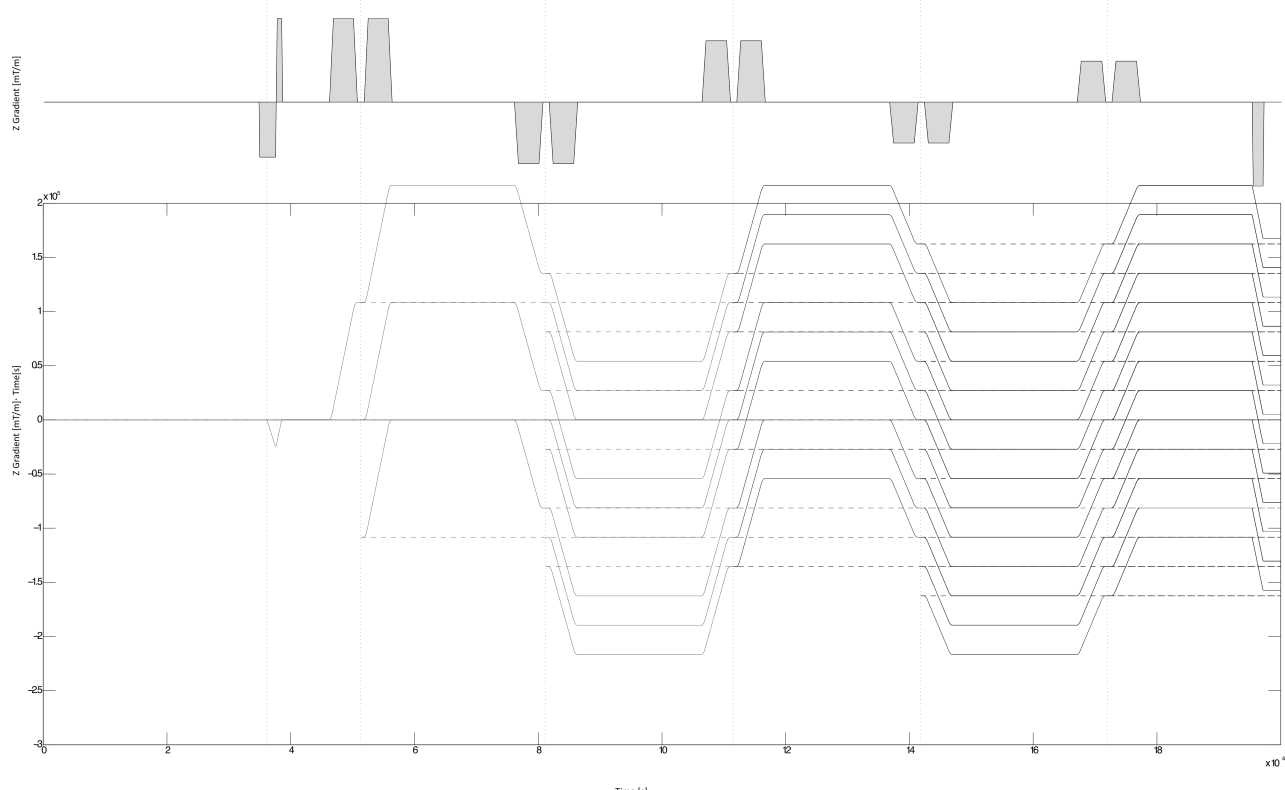

(b) Nonequal spoiling scheme

Figure 3.4: Phasegraph simulations of different spoiling schemes on the z-axis in a multi echo spin echo experiment. 


\section{$3.2{ }^{17} \mathrm{O}$ imaging}

As mentioned before, the use of ${ }^{17} \mathrm{O} \mathrm{MRI}$ provides the opportunity to perform localised measurements of $\mathrm{CMRO}_{2}$ in different brain regions in a voxelwise manner by means of signal intensity changes of $\mathrm{H}_{2}^{17} \mathrm{O}$. This opens up many possibilities such as studying healthy brain metabolisms, but can also be of enormous diagnostic value for the detection of pathological processes.

Ultrashort echo time (UTE) sequences such as flexible twisted projection imaging (fTPI) [70] or density-adapted 3D radial projection imaging (3D-DAPR) [97] provide appropriate signal acquisition methods, which are suited for the short relaxation times $\left(\mathrm{T}_{2}^{*} \approx 2 \mathrm{~ms}\right)$ of ${ }^{17} \mathrm{O}[61,97]$.

During the work for this thesis a fTPI (see section 3.4.1) sequence was used for phantom and in vivo ${ }^{17} \mathrm{O}$ imaging at a $9.4 \mathrm{~T}$ whole body human scanner and a $9.4 \mathrm{~T}$ small animal scanner. Results can be found in section 4.2.1. 


\subsection{Quantitative Imaging}

\subsubsection{Motivation}

A detailed knowledge of the quantitative water content is absolutely necessary to quantify $\mathrm{CMRO}_{2}$ on the basis of ${ }^{17} \mathrm{O}$ measurements, as mentioned in section 3.1.2. Atkinson et. al [61] used sodium imaging to identify white and grey matter in the brain and calculated tissue mass according to these measurements. Along that it is not a direct measure of tissue mass there is the need for an additional ${ }^{23} \mathrm{Na}$ RF coil and lengthy acquisition times compared to ${ }^{1} \mathrm{H}$ imaging methods. Quantitative $\mathrm{H}_{2} \mathrm{O}$ values are a direct measure and thus quantitative water mapping the method of choice to calibrate the acquired $\mathrm{CMRO}_{2}$ by means of ${ }^{17} \mathrm{O}$ imaging modalities.

Additionally accurate quantitative imaging of the human brain in clinical acceptable measurement times offers a wide range of applications for diagnostics. Commonly, only $T_{1}, T_{2}$, $T_{2}^{*}$ or proton density weighted images are acquired. These images do not offer the possibility to compare sets of images acquired on different scanners, with different parameters or at different time points. Quantification or quantitative analysis is not possible, because the contrast of the images depends on many different parameters.

Fast and accurate $T_{1}$ mapping of the human brain provides many opportunities for diagnostic use. For example, it has been shown that $T_{1}$ values differ within the globus pallidus, the caudate nucleus, and the posterior limb of the internal capsule of patients suffering from hepatic encephalopathy, a liver disease with neuropsychiatric consequences [98].

Since the acquired signal is dependent on $T_{1}, T_{2}, T_{2}^{*}$ and proton density (which represents more or less the water content) quantification of $\mathrm{H}_{2} \mathrm{O}$ can only be done, if all other parameters are taken into account. Neeb et al. [90] presented a method for fast quantitative mapping of absolute water content in vivo, which uses the TAPIR sequence which in turn is described in section 3.3.2.2. An imaging modality for estimating $T_{2}^{*}$ relaxation rates is described in section 3.3.3.2. The aforementioned method for water content mapping based on the TAPIR sequence was further enhanced and newly improved corrections for $B_{1}^{-}$inhomogeneities [10] were applied. 


\subsubsection{Mapping of Relaxation Times}

\subsubsection{Gold Standard - Spectroscopic Measurement of Relaxation Parameters}

\section{Longitudinal Relaxation Time $T_{1}$}

A spectroscopic inversion recovery sequence is gold standard for $T_{1}$ measurement. This sequence consists of a $\pi$ pulse, which inverts the equilibrium magnetisation and a subsequent $\pi / 2$ pulse after the inversion time, $\mathrm{TI}$, for excitation followed by a readout. $\mathrm{Tl}$ is defined as the time between the inversion and the excitation and has to be varied several times in order to make a $T_{1}$ fit possible. Assuming complete inversion, the longitudinal magnetisation right after the inversion pulse is given by:

$$
M_{z}\left(0^{+}\right)=-M_{0}
$$

where $M_{0}$ is the equilibrium value. During the $\pi$ and the $\pi / 2(0<t<\mathrm{TI})$ pulse the magnetisation relaxes to

$$
M_{z}(t)=M_{0}\left(1-2 e^{-t / T_{1}}\right) .
$$

After tipping the longitudinal magnetisation into the transverse plane $(t>\mathrm{TI})$, the magnitude of the transverse magnetisation is given by

$$
M_{\perp}(t)=\left|M_{0}\left(1-2 e^{-\mathrm{TI} / T_{1}}\right)\right| e^{-(t-\mathrm{TI}) / T_{2}^{*}} .
$$

The whole measurement is repeated $n$ times with $n$ different inversion times $\mathrm{TI}$ and $T_{1}$ can then be fitted from the data. The time between the acquisitions should be around $5 \cdot T_{1}$ to achieve full recovery and thus to start at the thermal equilibrium magnetisation $M_{0}$.

\section{Tranverse Relaxation Time $T_{2}$}

The transverse relaxation time constant $T_{2}$ can be measured by a spin echo experiment which is described in detail in chapter 2.3.3.1. The demodulated signal at the spin echo (at time TE) is

$$
s(\mathrm{TE}) \propto \omega_{0} e^{-\mathrm{TE} / T_{2}} \int d^{3} r B_{\perp}(\vec{r}) M_{\perp}(\vec{r}, 0) .
$$

Varying the echo time ( $T E^{\prime}>\mathrm{TE}$ ) leads to

$$
s\left(\mathrm{TE}^{\prime}\right) \propto \omega_{0} e^{-\mathrm{TE}^{\prime} / T_{2}} \int d^{3} r B_{\perp}(\vec{r}) M_{\perp}(\vec{r}, 0) .
$$


Combining equations (3.12) and (3.13), the spin-spin decay constant can be calculated from the two echo times TE and $T E^{\prime}$ utilising:

$$
T_{2}=\frac{\mathrm{TE}^{\prime}-\mathrm{TE}}{\ln \left(\frac{s(\mathrm{TE})}{s\left(\mathrm{TE}^{\prime}\right)}\right)} .
$$

To reach a better accuracy, more data points can be sampled. Again, the time between the different acquisition should be long in comparison to $T_{1}$ to achieve full recovery of the magnetisation.

\subsubsection{TAPIR sequence}

The TAPIR sequence ( [99-102], depicted in Figure 3.5) is a distortion free and accurate method to estimate high resolution $T_{1}$ maps. The TAPIR sequence is based on the LookLocker approach [103] and consists of a magnetisation preparation module followed by a segmented EPI readout. It allows a flexible selection of sample coverage and number of time points acquired on the recovery curve. Without the use of any acceleration method, it is too slow for whole brain coverage in clinical use. Recently it has been shown that the accuracy of Look-Locker based $T_{1}$ measurements can be increased throughout the use of an adiabatic fast passage (AFP) inversion pulse [32].

A short summary of the loop structure shall be given here. The innermost loop, the socalled "slice" loop, repeats the acquisition of one or - depending on the EPI-factor - more lines through all slices in one time-point. The "time-point" loop repeats the slice loop for all time-points. With this approach it is possible to acquire data of a given set of slices throughout the whole $T_{1}$ relaxation process. The outermost loop, the "line" loop, comprises the magnetisation preparation module and both of the other loops. It is called $k_{\max _{y}} /$ (EPI-factor $\cdot$ iPAT-factor) times. The nonselective magnetisation preparation module consists of a $90^{\circ}$ saturation pulse, a preparation delay time, $\tau$, (which is not shown to scale in Figure 3.5) followed by an inversion pulse. The saturation pulse and the delay $\tau$ are inserted to allow for a shorter acquisition time than allowed by an inversion recovery module. The behaviour of the relaxation curve is changed in such a way that a shorter sampling of the curve is sufficient. The next acquisition can start right after sampling the last time point and a pause of approximately $5 T_{1}$ can be avoided. The magnetisation preparation module is followed by a train of slice-selective excitation pulses and a segmented EPI-like readout for each slice. In a Look-Locker sequence the measured signal intensity $S$ is given by [103]

$$
S=M_{0} m \sin (\alpha)
$$




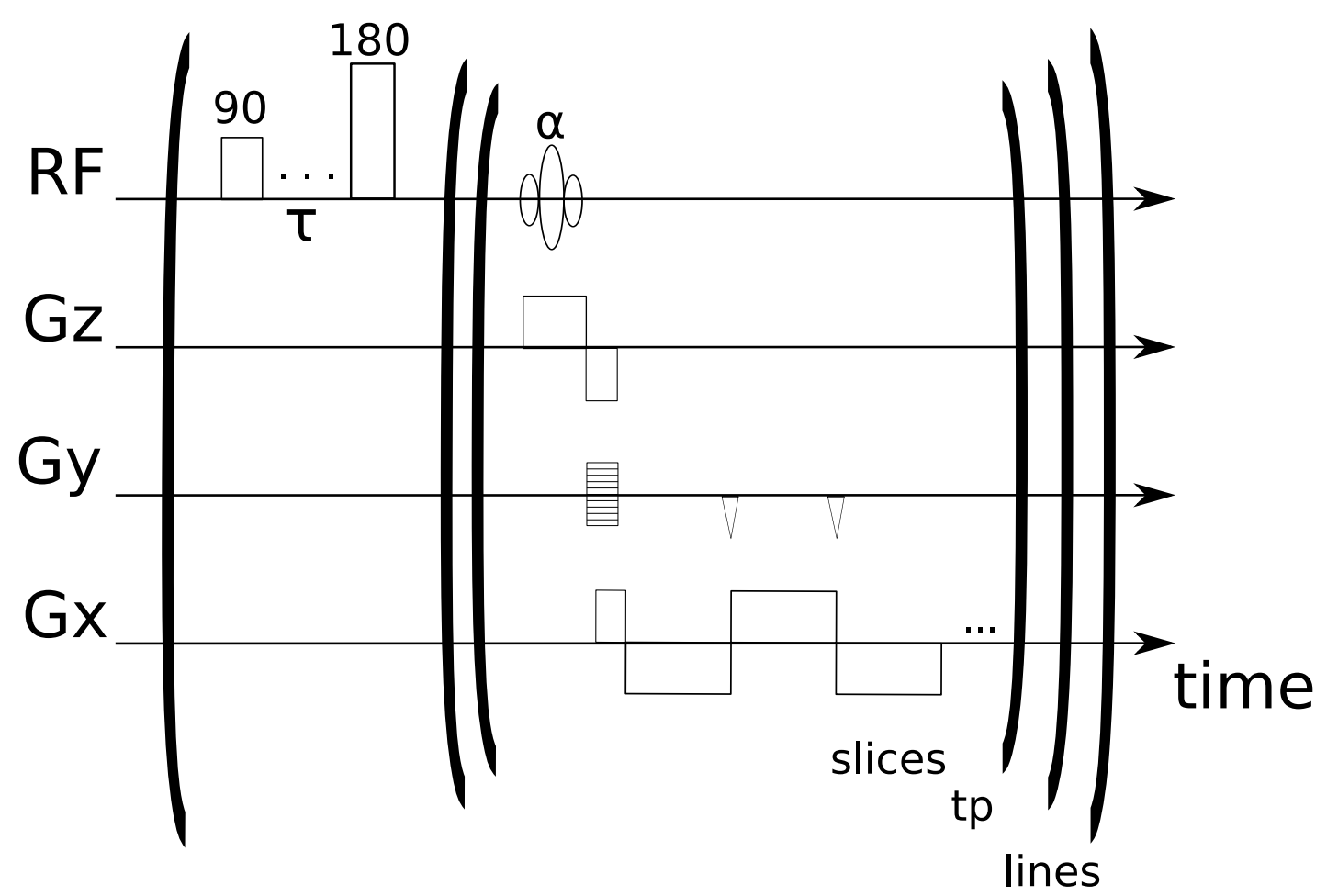

Figure 3.5: TAPIR sequence diagram with rectangular inversion. Time $\tau$ between $90^{\circ}$ and $180^{\circ}$ is not shown to scale.

with

$$
m=m_{\infty}-\left(m_{\infty}-m_{1}\right) \exp \left(-\frac{t-t_{n}}{T_{1}^{*}}\right)
$$

and

$$
\frac{1}{T_{1}^{*}}=\frac{1}{T_{1}}-\frac{\ln (\cos (\alpha))}{T R}
$$

where $m_{\infty}$ stands for the magnetisation in the steady state, $m_{1}$ for the initial magnetisation and $t_{n}$ for the time between the inversion and the first acquisition of slice $n$. 


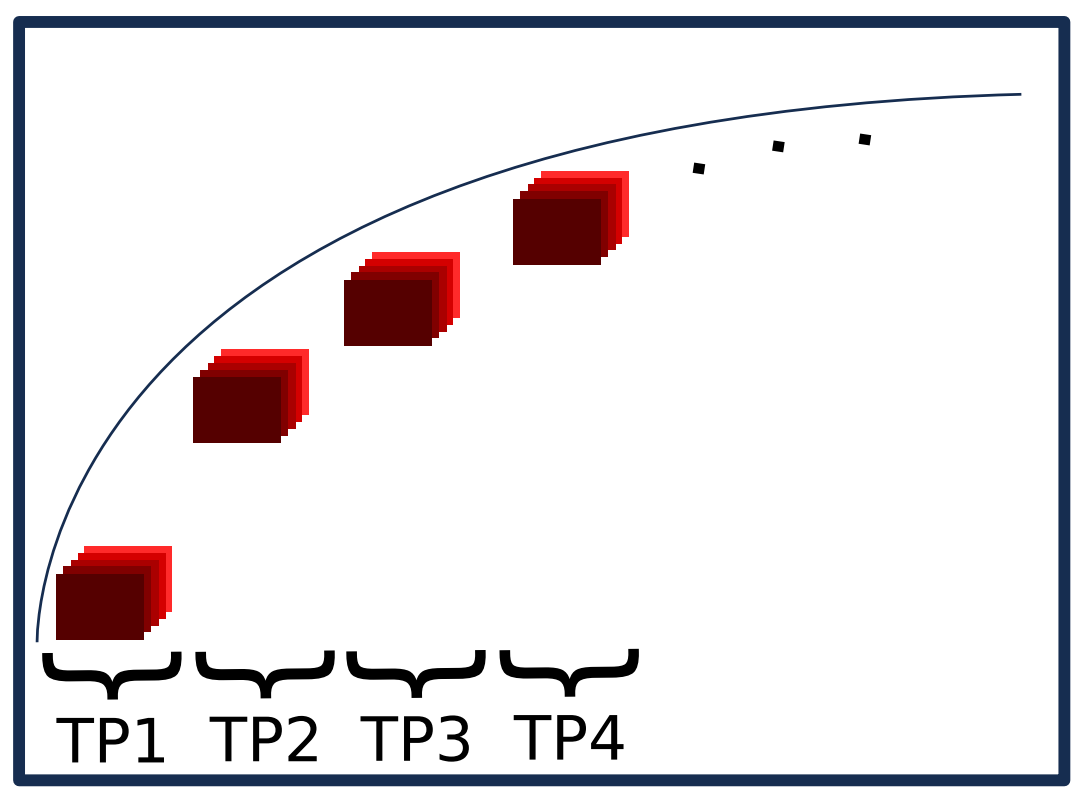

Figure 3.6: Schematic draft of the recovery of the MR signal which follows the inversion pulse. Sampling of the curve throughout all $n$ slices (red rectangles) and time-points is shown at the bottom of the Figure. TPn denotes the $n$-th timepoint.

Applying a large number of excitation pulses in succession, the longitudinal magnetisation $m_{z}(t)$ can be expressed as

$$
m_{z}(t)=m_{0}^{*}-\left(\left(m_{0}\right)+m_{0}^{*}\right) \cdot \exp \left(-\left(\frac{t}{T_{1}^{*}}\right)\right)
$$

where $m_{0}$ is the equilibrium magnetisation and $m_{0}^{*}$, the apparent equilibrium magnetisation, which can be expressed - for $T R \ll T_{1}^{*}$ - by $m_{0}^{*}=m_{0}\left(\frac{T_{1}^{*}}{T_{1}}\right)$.

Using Equation (3.18) and $A=m_{0}^{*}$ and $B=m_{0}+m_{0}^{*}, T_{1}$ can be calculated using

$$
T_{1}=T_{1}^{*}\left(\frac{B}{A}-1\right) .
$$

If the magnetisation preparation module is used, the equilibrium magnetisation is not the initial point and $A=m_{0}^{*}$ and $B=m_{0}\left(1-\exp \left(\frac{\tau}{T_{1}}\right)\right)+m_{0}^{*}$ have to be modified as fitting parameters. The voxelwise fitting for $M_{0}, \alpha$ and $T_{1}$ results in a set of quantitative maps. As shown by Zaitsev et al [104], the accuracy of the measurement depends largely on a well-chosen magnetisation preparation delay time $(\tau)$. It should be at least $\sim 1.5 \cdot T_{1}$, and for $\tau>2.5 \cdot T_{1}$ the accuracy does not change significantly. 
Inversion Efficiency Measurement

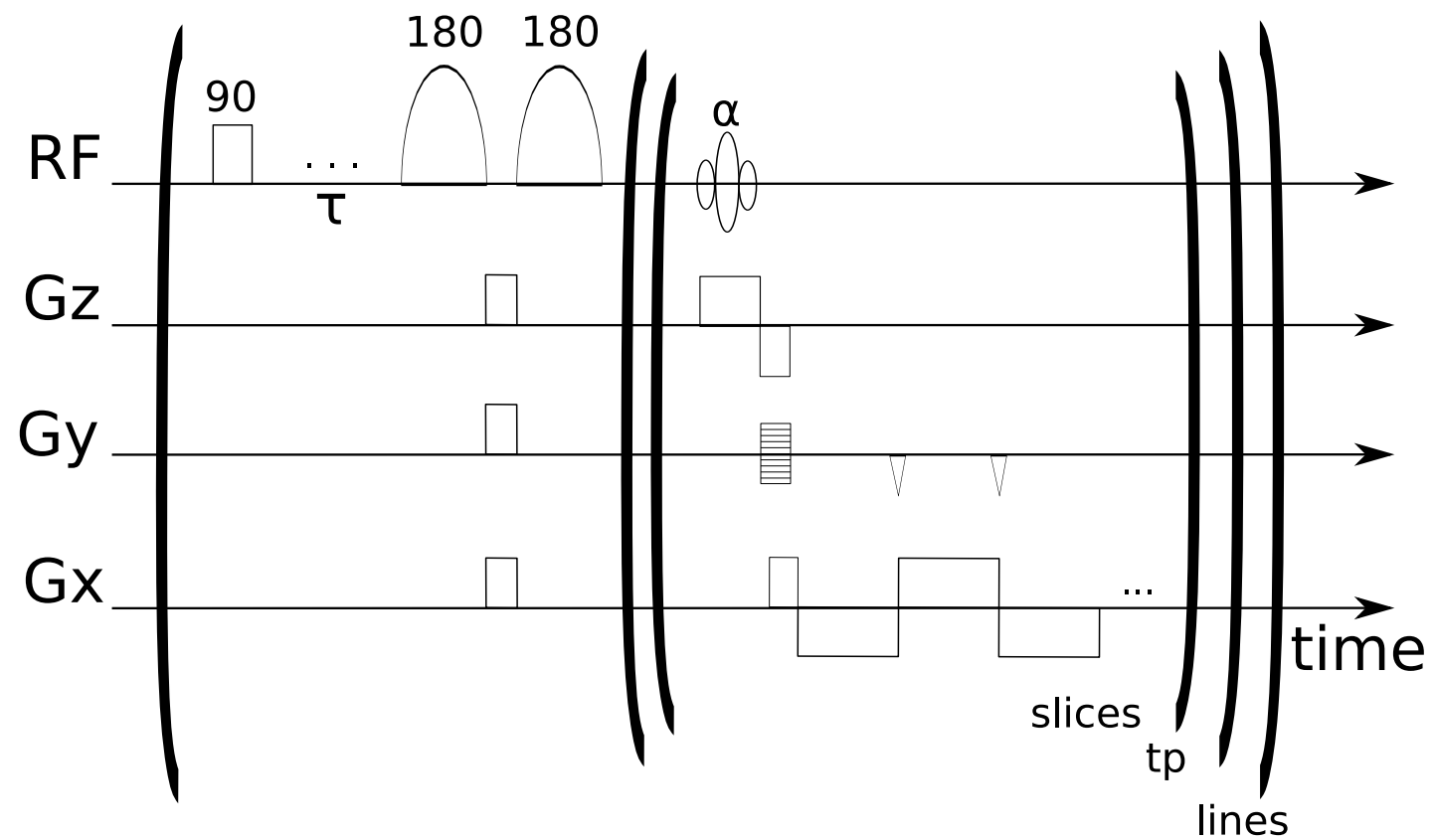

Figure 3.7: Sequence diagram of the inversion efficiency measurement. The two inversion pulses are played out in one go while in the second measurement these pulses are omitted.

The efficiency of the $180^{\circ}$ inversion pulse suffers from $B_{1}^{+}$inhomogeneities. Consequently, it is not homogeneously distributed over the measured volume. The inversion efficiency (IE) of the pulse used can be measured and has been described extensively in the literature $[102,104]$. For the sake of completeness, a short overview is given here.

The inversion efficiency measurement comprises two separate acquisitions of corresponding slices. The first experiment consists of the same saturation pulse and preparation delay time, $\tau$, as in the TAPIR sequence. Thereafter, two inversion pulses followed by an acquisition of one or more lines (corresponding to the chosen EPI factor) in $k$-space are performed. The second measurement applies no inversion pulse. Right after the preparation delay time, $\tau$, the same $k$-space lines as in the first experiment are acquired. The resulting longitudinal magnetisation after the $90^{\circ}-\tau$ module can be described for each acquired line by

$$
m=1-\exp \left(-\left(\frac{\tau}{T_{1}}\right)\right) .
$$

As mentioned above, this module is followed by a $180^{\circ}-$ Spoil $-180^{\circ}$ module and multislice acquisition as implemented in the TAPIR sequence. At time $t_{n}$ ( $n^{\text {th }}$ slice acquisition), the 
residual longitudinal magnetisation follows

$$
m=K\left(\exp -\left(\frac{\delta}{T_{1}}\right)\left(1+K\left(1-\exp \left(-\left(\frac{\tau}{T_{1}}\right)\right)\right)\right)-1\right) \exp \left(-\left(\frac{t_{n}}{T_{1}}\right)\right)
$$

where $\delta$ is the time required for spoiling between the inversion pulses to crush residual transverse magnetisation, and the inversion efficiency equals $K$. If the $180^{\circ}-S p o i l-180^{\circ}$ module is not applied, the magnetisation is given by

$$
m=1-\exp \left(-\left(\frac{\tau}{T_{1}}+\frac{t_{n}}{T_{1}}\right)\right) \text {. }
$$

The ratio of these two signals is included into the fitting process according to [104]

$$
\frac{s^{\prime}}{s}=\frac{1-\left(1-K\left(\exp \left(-\delta / T_{1}\right)\left(1+K\left(1-\exp \left(-\tau / T_{1}\right)\right)\right)-1\right)\right) \exp \left(-t_{n} / T_{1}\right)}{1-\exp \left(-\frac{\tau}{T_{1}}-t_{n} / T_{1}\right)}
$$

which is a quadratic equation regarding the inversion efficiency $K$.

This voxelwise fixed ratio needs to be included into the fitting procedure according to Eq. (3.23) because the IE depends on the $T_{1}$ of the corresponding voxel. 


\subsubsection{Water Mapping}

Water content in the human body is tightly regulated. The non-invasive quantification of water content allows one to improve diagnostics and to follow patient treatments in longitudinal studies in various diseases. However, as mentioned earlier (see section 3.1.2), a ground truth of the water content signal is needed to achieve quantitative $\mathrm{CMRO}_{2}$ values based on ${ }^{17} \mathrm{O}$ imaging methods.

As described in the literature [105-108], many pathological conditions are accompanied by a global or local increase in water content. These changes largely depend on the underlying pathology. For example hepatic encephalopathy (HE) results in global water content changes of about $\sim 1 \%-3 \%$ [109] while brain tumour patients often show an increased water content primary in white matter up to $20 \%$ [107].

\subsubsection{1 $M_{0}$ Maps}

Common MR sequences are based on proton imaging. Thus, an $M_{0}$ map can provide information about the water content of the imaged object. Due to the rather short $T_{2}^{*}$ of hydrogen embedded in macro or bound water molecules, standard MRI methods almost entirely acquire signals of free water protons since the larger part of the magnetisation of such fast decaying species is already dephased at the shortest possible echo time with common imaging sequences. [110] Hence, in the presented work, water mapping means the measurement of free water. In vivo free water is mostly present in intra- and extracellular fluid.

As stated in chapter 3.3.2.2, during the postprocessing of the TAPIR signal data, a three parameter fit for $M_{0}, T_{1}$ and the flip angle $\alpha$ is performed. The resulting $M_{0}$ map can be used as a basis for quantitative water mapping.

The TAPIR fitting routine is rather stable with regard to $T_{1}$, but it is unstable between $M_{0}$ and the flip angle $\alpha$. It can be assumed that the $B_{1}$ field, which is directly proportional to the flip angle map, does not have large variation on small distances [111]. Thus a median filter with a radius of 5 voxels is applied to the resulting flip angle map. With this filtered flip angle map another 2 parameter fit $\left(T_{1}\right.$ and $\left.M_{0}\right)$ is performed. The resulting $M_{0}$ map is not as noisy as before and the $T_{1}$ map is quantitatively unaffected and remains the same such as the resulting $T_{1}$ map of the first 3 parameter fitting procedure.

\subsubsection{2 $T_{2}^{*}$ Correction}

In MR imaging sequences, TE is usually about a few milliseconds. During this time, $T_{2}^{*}$ relaxation of the signal takes place. To obtain quantitative data, the $M_{0}$ maps have to be 
corrected for $T_{2}^{*}$ contamination voxelwise. The required $T_{2}^{*}$ maps were accessed using slice selective 2D MR imaging sequence called quantitative T2 $_{2}^{*}$ image (QUTE) [89-91], which is a multislice multi-echo gradient echo sequence.

Using multi echoes at different echo times, TE, the $T_{2}^{*}$ relaxation constant can be fitted to the signal intensity. Due to the exponential decay of the signal intensity with the decay time, $T_{2}^{*}$, between excitation and readout, the correction

$$
S_{0_{T_{2}^{*} \text {-corrected }}}=S_{0}(\mathrm{TE}) \cdot \exp \left(\frac{\mathrm{TE}}{T_{2}^{*}}\right)
$$

has to be applied to the $M_{0}$ map voxelwise.

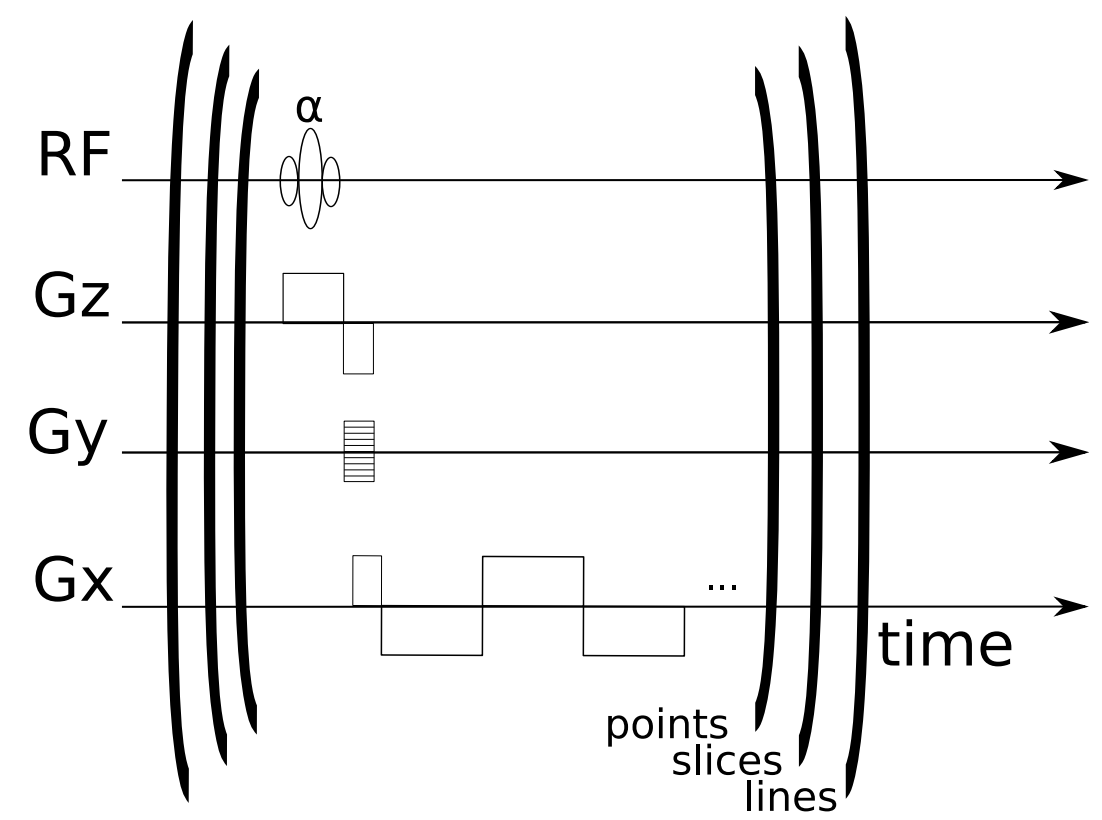

Figure 3.8: QUTE MR pulse sequence diagram [89-91]. Following a slice-selective pulse, the signal is phase encoded for the highest line of $k$-space and several gradient echoes are read out. This loop is repeated until the required number of echoes have been acquired.

\subsubsection{Receiver Coil Sensitivity Correction}

For in vivo human brain imaging, transmission and reception is often performed by different coils. Nowadays, parallel head coil arrays with several coil elements are used for signalreception as mentioned in section 2.3.7 and the static, installed body coil is used for signalexcitation. The sensitivity of both coils differ resulting in a different $B_{1}^{-}$weighting of every calculated $M_{0}$ map.

In earlier times and on lower field strengths such as $1.5 \mathrm{~T}$, the same sequence with the same parameters was measured twice. In a first acquisition the body coil was used for 
transmission and reception of the signal and in a second measurement, the body coil was used to excite the signal and the head coil for receiving the signal. With this information Lorentz's reciprocity theorem was used for correction but this is not sufficient at $3 \mathrm{~T}$ and above where the $B_{1}^{-}$homogeneity is reduced [112-115].

To overcome this issue an unified segmentation [116] was proposed $[117,118]$, which only holds for in vivo and human brain and may fail in presence of pathological changes. As shown by Abbas et al. [10] in 25 healthy subjects, the well-known correlation existing between tissue $T_{1}$ and proton density (PD) can be used to estimate the receive bias field properly.

If one defines the residual nonuniformity in the PD map:

$$
\mu=\left(\frac{\mathrm{R} B_{1}^{+}}{B_{1}^{-}}\right)
$$

which is still present after the correction by the reciprocity theorem one can use the correlation of $T_{1}$ and PD [119] in a way that

$$
\mathrm{PD}^{-1}=C_{0}+C_{1} T_{1}^{-1}
$$

with coefficients $C_{0}$ and $C_{1}$. Defining $Q=\frac{C_{1}}{C_{0}}$ and treating these correlation coefficients as spatial distributed this leads to a PD contrast (PDC)

$$
\operatorname{PDC}(r)^{-1}=\mu(r) C_{0}(r)\left(1+Q(q) T_{1}(r)^{-1}\right)
$$

To reduce the probability of observing $T_{1}$ effects in the regions which are not explained by a PD variation, the region $\Omega$ is restricted to homogenous $T_{2}^{*}$ values in such way $\Omega_{I}=_{\text {def }}$. $\left\{r ; T_{2}^{*}(r) \in I\right\}, I$ being region within $T_{2}^{*}$ of $[50,60] \mathrm{ms}$, thus according to [10] we can assume $C_{0}$ and $C_{1}$ constant in such regions resulting in:

$$
\operatorname{PDC}(r)^{-1}=\mu(r) C_{0}\left(\Omega_{I}\right)\left(1+Q\left(\Omega_{I}\right) T_{1}(r)^{-1}\right),
$$

with $C_{0}\left(\Omega_{I}\right)$ and $Q\left(\Omega_{I}\right)$ being constants.

This technique allows assessing $\mu(r)$ for all voxels in $\Omega_{I}$ and can be extrapolated to a complete map by means of field map regularization. Details can be found in the literature $[10]$ and are beyond the scope of this thesis.

\subsubsection{Calibration to CSF}

Since the $M_{0}$ map is in arbitrary units it has to be rescaled to known values. Following $T_{2}^{*}$ - and receiver coil sensitivity correction as mentioned in section 3.3.3.3 a calibration 
of the $M_{0}$ map to the known water content of CSF is done. It is assumed to have the same water content as pure water. The CSF signal intensity was measured in the right and left lateral ventricles, by automatically ROI selection based on $T_{1}$ and $T_{2}^{*}$ information with $T_{1}>2900 \mathrm{~ms}$ and $T_{2}^{*}>500 \mathrm{~ms}$ [11]. Partial volume effects are minimised by erosion of the segmented volume. 


\subsubsection{Data Processing}

Initially, the TAPIR signal is processed. The resulting flipmap is median-filtered (for more details see section 3.3.3.1). With this median filtered flipmap, the TAPIR signal data is processed once again. Exponential fitting of the data acquired with the QUTE sequence results in a quantitative voxelwise $T_{2}^{*}$ map with the same resolution. This map has to be applied as a voxelwise correction (see section 3.3.3.2) as well.

This map is then corrected for receiver coil inhomogeneities as mentioned in section 3.3.3.3, which finally results in a quantitative map of water content. 


\subsection{Sequence Design}

\subsubsection{Twisted Projection Imaging - TPI}

Boada et al. [70] presented an efficient 3D sampling strategy for the acquisition of fast relaxing nuclei such as sodium $\left({ }^{23} \mathrm{Na}\right)$ or oxygen $\left({ }^{17} \mathrm{O}\right)$. In standard Cartesian sampling the echo time is limited by the read dephaser and the time of the readout gradient until the center of $k$-space is reached. At low readout bandwidths, which are necessary to achieve sufficient SNR with fast relaxing nuclei, this leads to typical echo times of approximately $3 \mathrm{~ms}$ even if an asymmetric readout (echo does not appear in the centre of the readout but already at e.g. $\left.\frac{1}{4}\right)$ is used. This can be overcome by the use of projection imaging $(\mathrm{PI})$ methods $[120,121]$. By using $\mathrm{PI}$, a short echo time is achieved due to the absence of phase encoding and slice selection. These methods are very efficient in imaging fast relaxing species, but they result in long acquisition times caused by a poor sampling efficiency especially at the edge of the 3D $k$-space.

One way to overcome the inefficient sampling is to use spiral imaging in $2 \mathrm{D}$ or $3 \mathrm{D}$ equivalent cones but the $k$-space trajectories of these sampling schemes close to the origin are limited due to gradient slew rate. As suggested by Boada [70], a mixture of $\mathrm{PI}$ and cone imaging can be implemented. A typical sampling scheme of this so-called twisted projection imaging method and the accompanying pulse sequence diagram are shown in Figure 3.9. 


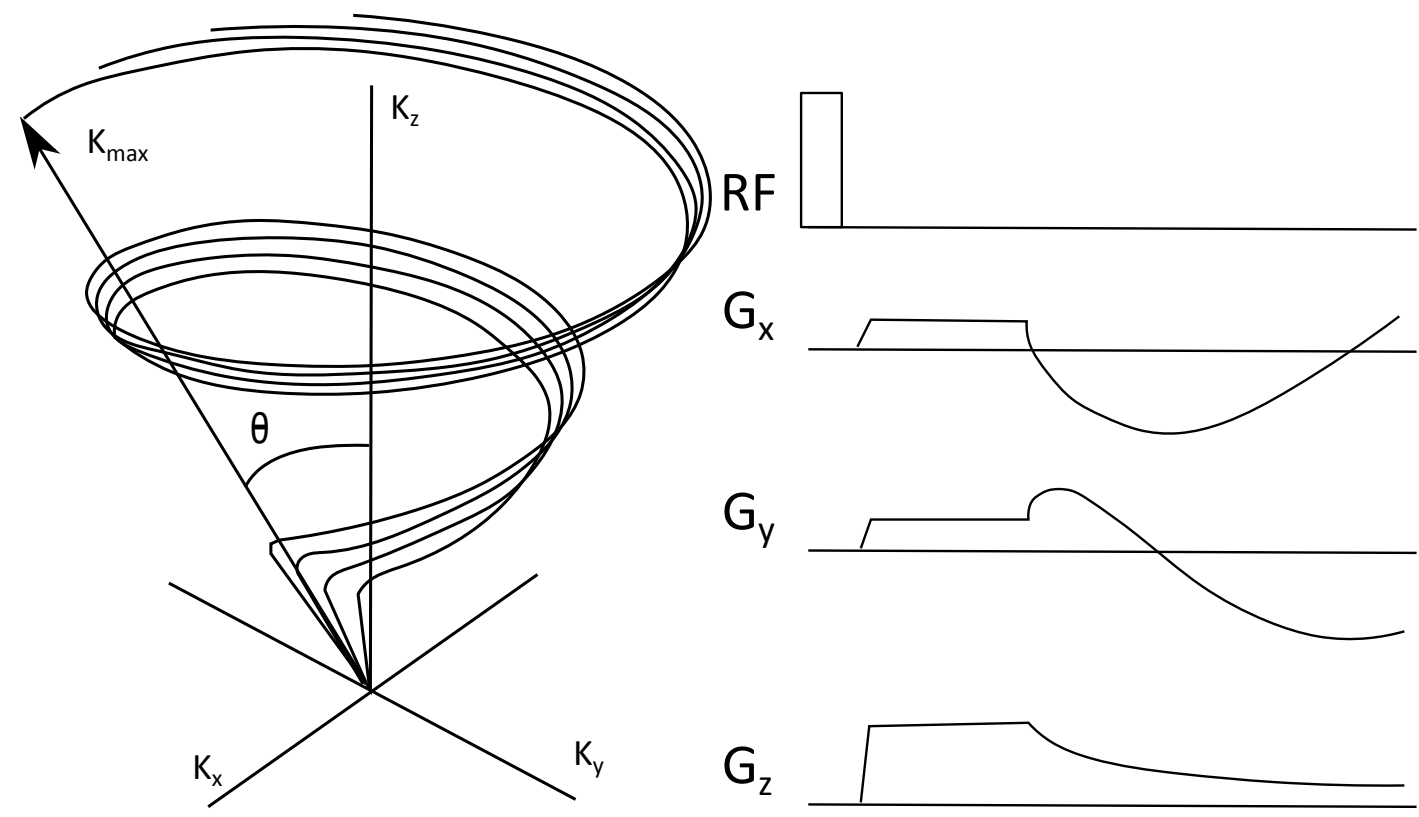

(a) Depiction of the sampling scheme in twisted- (b) Sequence diagram of twisted-projection-imaging. projection-imaging.

Figure 3.9: Depiction of TPI sequence diagram and corresponding $k$-space trajectory. Adapted from [70].

The scheme was developed as follows

1. Surface of a sphere with the radius $K_{\max }$ is divided into even numbers $N_{R}$ of rings which are separated by a distance equal to the inverse of the desired FoV. Each ring defines a particular cone at an angle $\Phi_{0}$.

2. The $k$-space trajectory of each cone departs from the origin in a radial line up to a fraction $p$ of $K_{\max }$. At that particular point the $k$-space trajectory starts to twist in such way that the sample density is maintained until $K_{\max }$ is reached. 


\subsection{2 $T_{1}$ Mapping with TAPIR}

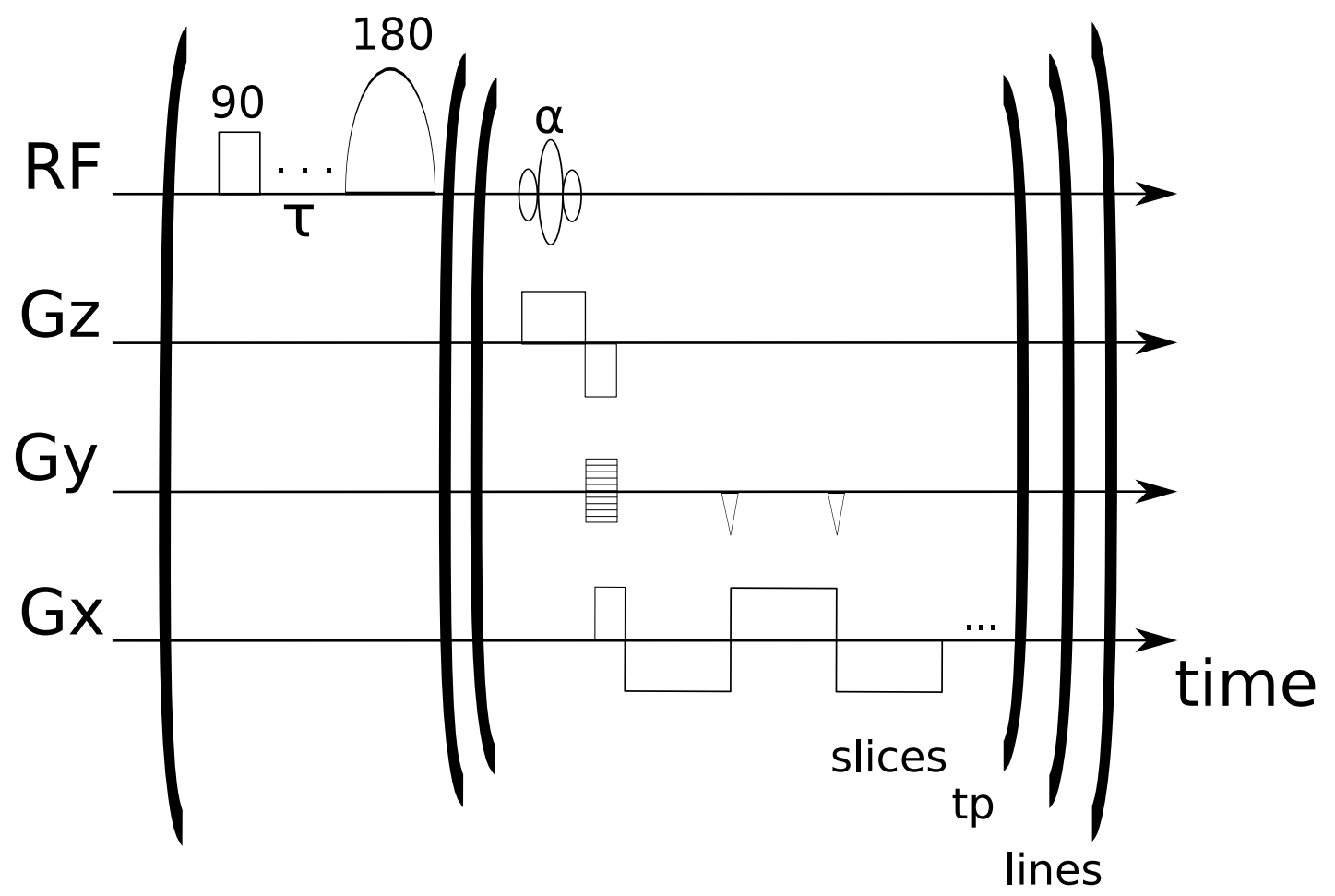

Figure 3.10: TAPIR sequence diagram with adiabatic inversion pulse

The original TAPIR sequence [99-102], which is depicted in Figure 3.5, allows a fast and accurate measurement of $T_{1}$ relaxation times. Since time is a limiting factor in the clinical application of MR sequences, it is a common need to make the sequence faster. This is achieved using parallel imaging in addition to the EPI-like readout, which was mentioned in chapter 2.3.7. A gain in accuracy of the $T_{1}$-mapping procedure can be achieved with the use of adiabatic inversion pulses [32]. The use of ETS (see section 2.3.8.1) makes the use of EPI factors less prone to image artefacts.

\subsubsection{Inversion Efficiency and Adiabatic Inversion Pulses}

As considered in chapter 2.3.5.3, inhomogeneities in the excitation field $B_{1}^{+}$results in an undesired excited flip angle effect. Imperfect inversion of the longitudinal magnetisation prior to the acquisition, gives rise to a misinterpretation of the $M_{0}$ value. Because of the way in which $M_{0}$ is included into the equation for $T_{1}(3.15)$, this would result in an underestimation of the $T_{1}$ value. As mentioned in chapter 2.3.6, employing a hyperbolic secant inversion pulse instead of a rectangular inversion pulse gives rise to a more homogeneous inversion 
even over a large volume (depicted in Figure 3.10). The newly used inversion pulse is described in chapter 2.3.6.2. The used parameters were $\mu=5.0$ and $\beta=4.5$ with a pulse duration $T_{p}=8.192 \mathrm{~ms}$. Following Deichmann [32], the amplitude of the AFP pulse with a pulse duration, $t_{\text {pulse }}$, should be set to 13.5 times the amplitude of a rectangular pulse with the same pulse duration, $t_{\text {pulse }}$.

\subsubsection{Parallel Imaging Combined with EPI Readout}

In addition to the adiabatic inversion pulse, which is used to get a better accuracy, parallel imaging - such as it is described in 2.3.7 - is implemented in the sequence. Using this, it is possible to speed up the acquisition by a factor of 2 without any significant penalties in accuracy. Combining this with a segmented EPI readout scheme, a gain of a factor of 10 in speed is feasible.

\begin{tabular}{cc}
\hline & acquisition time [min] \\
\hline TAPIR & $14: 30$ \\
TAPIR EPI3 & $5: 00$ \\
TAPIR EPI5 & $3: 00$ \\
TAPIR EPI3 iPAT2 & $2: 30$ \\
TAPIR EPI5 iPAT2 & $1: 30$ \\
\hline
\end{tabular}

Table 3.2: Acquisition times for different accelerated TAPIR sequences. Parameters: Number of slices $=8$, Slice thickness $=2 \mathrm{~mm}, T R=12 \mathrm{~ms}, 20$ time points, $\mathrm{TI}=20 \mathrm{~ms}, \mathrm{BW}=720 \mathrm{~Hz} / \mathrm{Px}$, $\alpha=25^{\circ}, \tau=2400 \mathrm{~ms}$, FoV read $=220 \mathrm{~mm}$; FoV phase $=167 \mathrm{~mm}$, in plane resolution: $1 \mathrm{~mm} \times 1 \mathrm{~mm}$. A comparison of accuracy and precision in phantom experiments can be found in section 4.1.2

Table 3.2 illustrates the breakdown of total acquisition time of a quantitative mapping process using the TAPIR sequence with a given set of exemplary parameters. Without any acceleration the total acquisition time for a $T_{1}$ map with volume coverage of 8 slices with a slice thickness of $2 \mathrm{~mm}$ is about $14: 30$ minutes ( $T R=12 \mathrm{~ms}$ and 20 time points (TP), $\mathrm{FA}=25^{\circ}, \mathrm{BW}=720 \mathrm{~Hz} / \mathrm{Px}$, resolution $1 \mathrm{~mm} \times 1 \mathrm{~mm} \times 2 \mathrm{~mm}$ ) and additional 2 minutes for an inversion efficiency measurement using an EPI factor of 5 ( 8 slices, TR $=12 \mathrm{~ms}$, $\mathrm{FA}=25^{\circ} \mathrm{BW}=720 \mathrm{~Hz} / \mathrm{Px}$, resolution $2 \mathrm{~mm} \times 2 \mathrm{~mm} \times 2 \mathrm{~mm}$ ). Using parallel imaging with an acceleration factor of 2 and an EPI-factor of 5 the acquisition time of the TAPIR sequence can be reduced to 1:30 minutes. This results in an effective total acquisition time for the TAPIR sequence of less than 12 seconds per slice. 


\subsubsection{QUIXOTIC}

The OEF and the $\mathrm{CMRO}_{2}$ are fundamental metabolic parameters of the brain indicating health and function. With the knowledge of section 3.1.4.1 enables calculation of OEF from known haematocrit $(\mathrm{Hct})$ and transverse relaxation rates $\left(\mathrm{T}_{2}\right)$ of venous blood. The most critical point is to acquire MRI signal of venous blood only. One way to calculate global OEF values based on signal from e.g. sagittal sinus (SS) only [15]. The main disadvantage of this technique is that it employs only one single global brain value. No localisation of the $\mathrm{OEF}$ and $\mathrm{CMRO}_{2}$ is possible.

To address this issue, a novel method called QUantitative Imaging of eXtraction of Oxygen and TIssue Consumption (QUIXOTIC) was introduced by Bolar et al. [12-14].

\subsection{Venular-Targeted Velocity-Selective Spin Labelling}

The key point in the QUIXOTIC measurement approach is the introduction of a so-called venular-targeted velocity-selective spin labelling (VT-VSSL), which allows for targetting of the MR signal to the postcapillary venular (PCV) blood compartment. This is achieved by applying velocity selective MR pulses to exploit different blood velocities in the vascular tree. The method relies on a uniformly forward flow from arteries, throughout the capillaries to the veins.

Data acquisition was done in two different steps, which creates a control and a tag image of the desired slice. Pairwise subtraction at each different $T E_{\text {eff }}$ eliminates signal from static tissue, CSF and non PCV blood and leaves a dataset comprised of PCV blood only. Bolar et al. adapted the QUIXOTIC sequence from a VS-ASL sequence [122] and use similar VS modules, which are depicted in Figure 3.11. The imaging sequence itself is shown in Figure 3.12 .

Since velocity is a time derivative of the position, it can be phase encoded by applying bipolar gradient fields or equivalently by unipolar pulses separated by a $180^{\circ} \mathrm{RF}$ refocusing pulse [123]. If this is accompanied by two excitation pulses of opposite phase $\left(90^{\circ}-\overline{90^{\circ}}\right)$, it results in a pulse train

$$
90^{\circ}-G-180^{\circ}-G-\overline{90^{\circ}} \text {. }
$$

If this magnetisation preparation module is applied to laminar flowing spins, it results in rephased spins flowing with a velocity slower than a certain cutoff velocity $V_{\text {cutoff, }}$ which can be calculated by $[124,125]$

$$
V_{\text {cutoff }}=\frac{\pi}{\beta}=\frac{\pi}{\gamma G \delta \Delta},
$$


where $\Delta$ represents the separation, $\delta$ the duration and $G$ the amplitude of the bipolar gradient pulses. As suggested in [124], a four RF a four-gradient pulses approach is used according as following

$$
90^{\circ}-G^{+}-180^{\circ}-G^{-}-G^{+}-180^{\circ}-G^{-}-\overline{90^{\circ}} \text {. }
$$

Simple but robust AFP pulses, as mentioned in section 2.3.6, were used by Bolar et al. for robust refocusing even at inhomogenous $B_{1}^{+}$and $B_{0}$ fields. To achieve lower SAR values, which enables shorter echo-spacing and/or longer $\mathrm{TE}_{\text {eff }} \mathrm{HSn}$ pulses (introduced in section 2.3.6.3 as well) were used in the used implementation. The used VT-VSSL module employed in this work is shown in Figure 3.11.
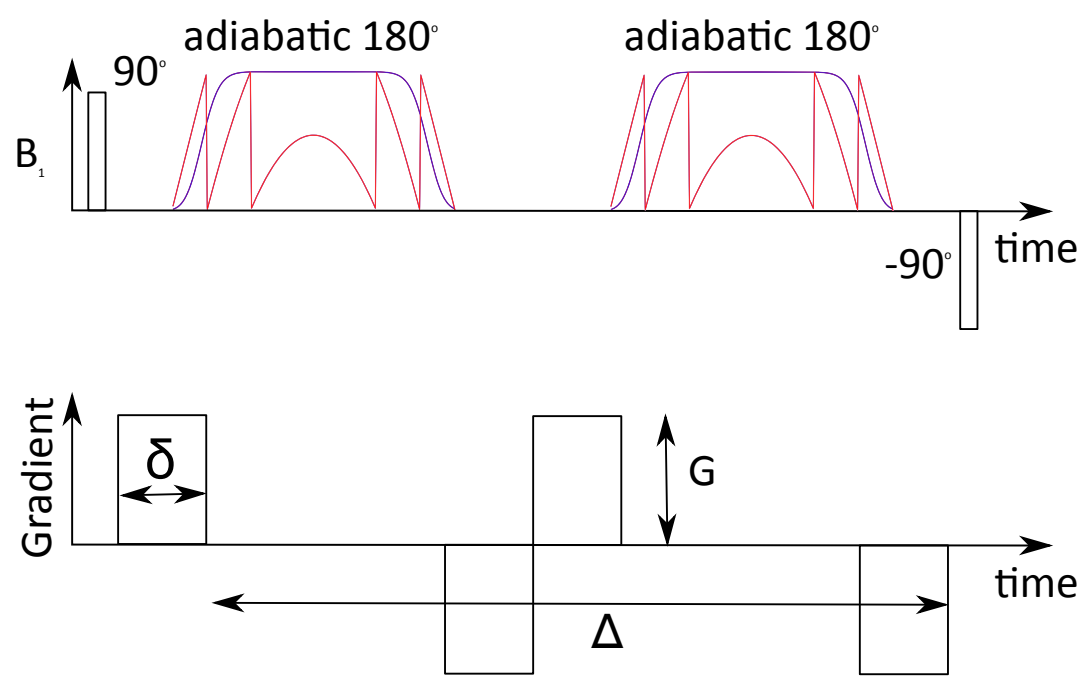

Figure 3.11: Depiction of the used velocity selective module with amplitude (blue) and phase (red) modulation of the used HSn pulses. Velocity weighting is defined by the timing of the gradient duration $\delta$, gradient separation $\Delta$ and gradient amplitude $\mathrm{G}$.

\section{Tag / Control}

As shown in Figure 3.12, the sequence consists of two different velocity selective (VS) modules (namely VS1 and VS2). Both modules are played out in tag and control mode. The only difference is inside of VS2. While a user-defined cutoff velocity $V_{\text {cutoff }}$ for both VS1 and VS2 is applied in tag mode, in control mode it is only applied in VS1 and disabled $\left(V_{\text {cutoff }}=\infty\right)$ in $\mathrm{VS} 2$. 


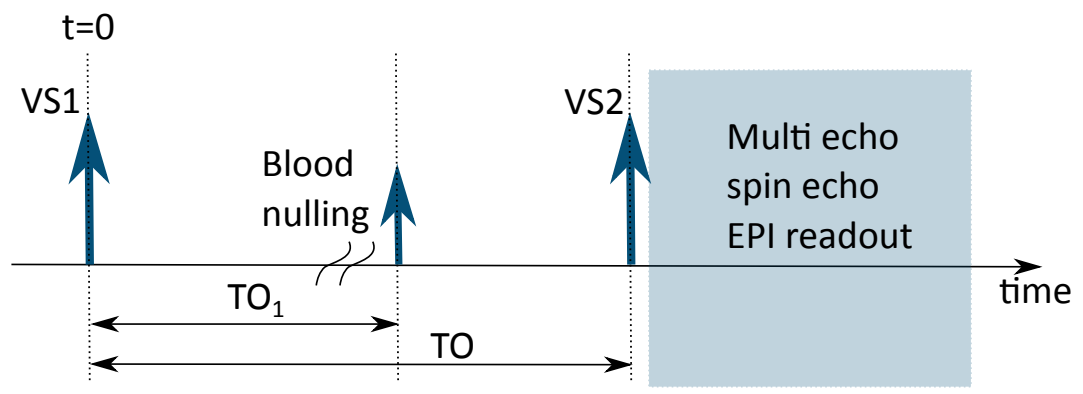

Figure 3.12: Schematic pulse sequence timing diagram of the QUIXOTIC / turbo QUIXOTIC sequence. The sequence runs once in control and once in tag mode. These differ in the velocity selective labelling in VS2.

At time TO1 an inversion pulse is included to compensate for adverse effects of $T_{1}$ relaxation which could potentially be introduced by unwanted arterial blood signal. A schematic representation of spin labelling is shown in Table 3.3. $T_{1}$ relaxation effects and arterial blood nulling via inversion at TO1 will be neglected in a first model.

At time $\mathrm{t}=0$, before VS1 is applied, all arterial, venous and capillary blood is relaxed. Applying of VS1 a strong velocity weighting with a low $V_{\text {cutoff }} \approx 1 \mathrm{~cm} / \mathrm{s}$ is achieved and only spins flowing with a velocity slower than $V_{\text {cutoff }}$ contribute to the signal. After the outflow time TO, which allows for blood flow of the prepared spins from capillaries to veins during which it is accelerated. At time $t=\mathrm{TO}$ the second preparation module VS2 is applied. In case of control mode, the velocity weighting is set to $\infty$, which implies no velocity weighting, and the resulting magnetisation originates from all spins which where labelled in VS1. If the tag mode is applied, another weighting with the same $V_{\text {cutoff }}$ is applied, which results in a dephasing of the spins which where accelerated to velocities faster than the desired $V_{\text {cutoff. }}$ This in turn represents the blood which resided in capillaries during VS1 and moved to larger veins. If a simple subtraction of the resulting images (Control-Tag) is performed the remaining signal originates from venous blood only, that is if flow is assumed to be directed from arteries to capillaries to veins. Signal originating from other sources such as tissue or CSF is eliminated via subtraction. While the original QUIXOTIC implementation had a T2prep module, as introduced by Botnar for improved coronary artery imaging [126], consisting of $90^{+\circ}-180^{\circ}-180^{\circ}-90^{-\circ}$ pulses, where the number of 180 pairs is varied, to

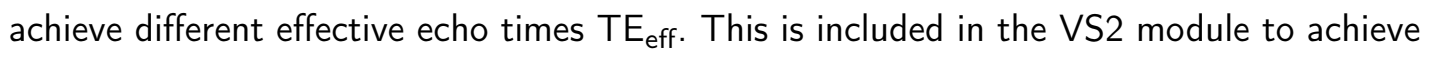
different $T_{2}$ weightings. The turbo QUIXOTIC approach is not using T2prep modules but a multi-echo, spin-echo readout as described in section 3.1.4.2.

\subsection{Measurement Setup}

First, segmented multi shot multi-echo, spin-echo imaging is performed on a phantom and quantitative $T_{2}$ values were compared to previously measured spectroscopic relaxation rates. 
Table 3.3: Idealized sketch of venular blood signal targeting. Dephased spins are labelled with dots, spins that can contribute to the signal with an arrow.

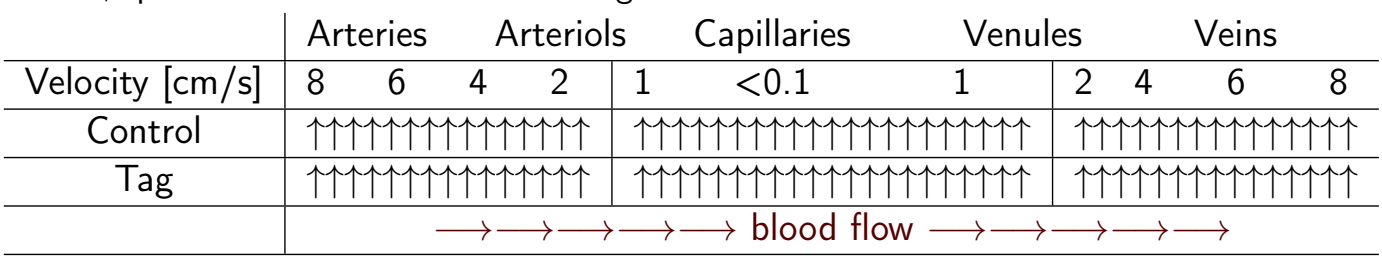

Before VS module I $\left(t=0^{-}\right)$

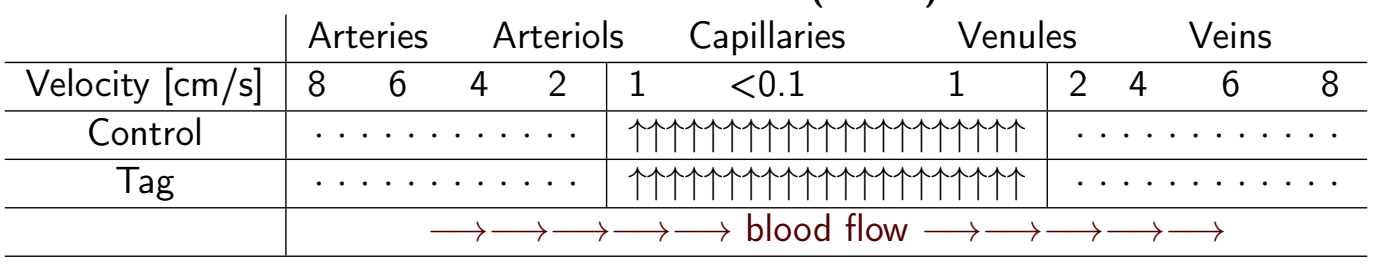

After VS module I $\left(t=0^{+}\right)$

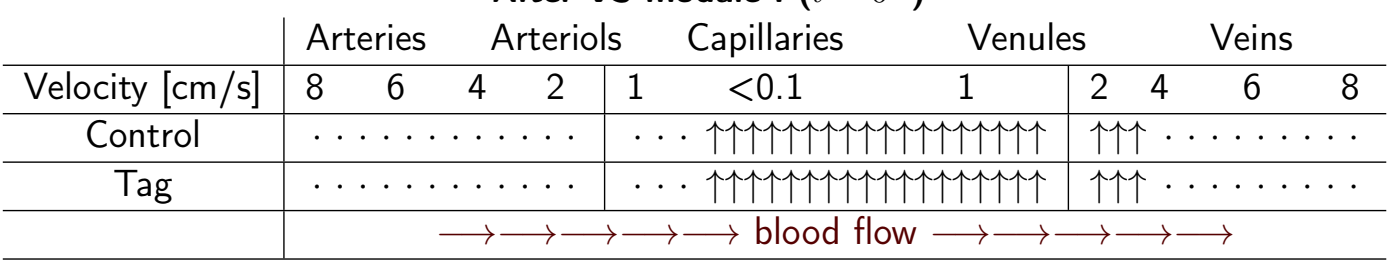

Before VS module II $\left(t=\mathrm{TO}^{-}\right)$

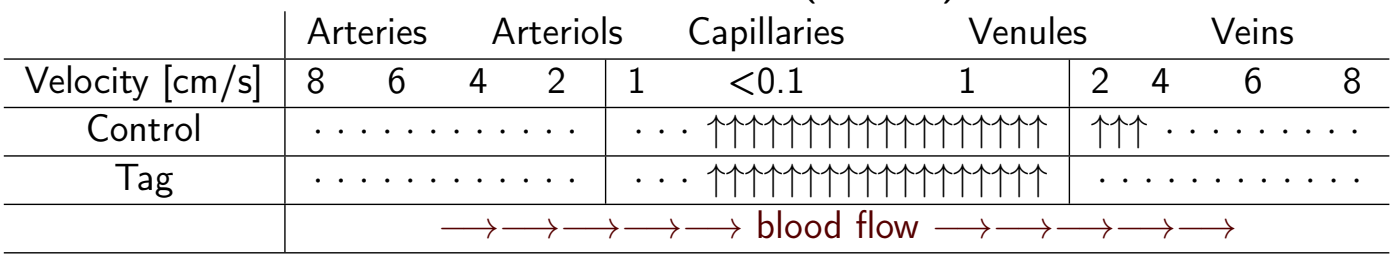

After VS module II $\left(t=T O^{+}\right)$

Secondly, quantitative $T_{2}$ relaxation rates of venous blood of four healthy volunteers were acquired in vivo with written, informed consent at a $3 T$ Tim-Trio MRI system (Siemens AG, Erlangen, Germany) with the use of the manufacturers 32 channel receive head array.

Measurements with the newly improved turbo QUIXOTIC variant by Möllenhoff et al [16], utilizing segmented multi-shot EPI, were carried out and compared to results of the traditional T2prep and published multiple-echo based variant. $Y_{v}$ values were calculated from quantitative venous $T_{2}$ values as published by Bolar and according to section 3.1.4.1.

The CBF was measured using a pseudo Continuous ASL (pCASL) [127] sequence which makes use of a 1.4 second labelling train of RF and magnetic gradient pulses to invert the magnetisation in the labelling slab. The position of the labelling slab was chosen on the basis of a quick time-of-flight angiography to ensure an optimal orientation and positioning of the carotid and vertebral arteries. The measured data was evaluated using the ASL 
data processing toolbox published by Wang et al. [128]. Directly before the MRI measurements, hematocrit was measured via finger prick blood samples using the Ultracrit device (Separation Technologies, Altamonte Springs, FL). All data postprocessing was performed similar to Bolar et al., which means in detail a pairwise subtraction of VS2-VS1 data for each $T E / T E_{\text {eff }}$ following a voxelwise exponential fit to achieve quantitative $T_{2}$ values. All unphysiological values (in that case $<0 \mathrm{~ms}$ and $>130 \mathrm{~ms}$ ) were removed via thresholding, outliers and voxels containing no information $(\mathrm{NaN})$ were then removed via the build in tools in Image J [129]. The resulting quantitative $T_{2}$ map were used to calculate $Y_{v}$ for each voxel which then can be used together with the CBF map, resulting from the $\mathrm{pCASL}$ measurements, to calculate a $\mathrm{CMRO}_{2}$ map. The data postprocessing is visualised in Figure 3.13 .

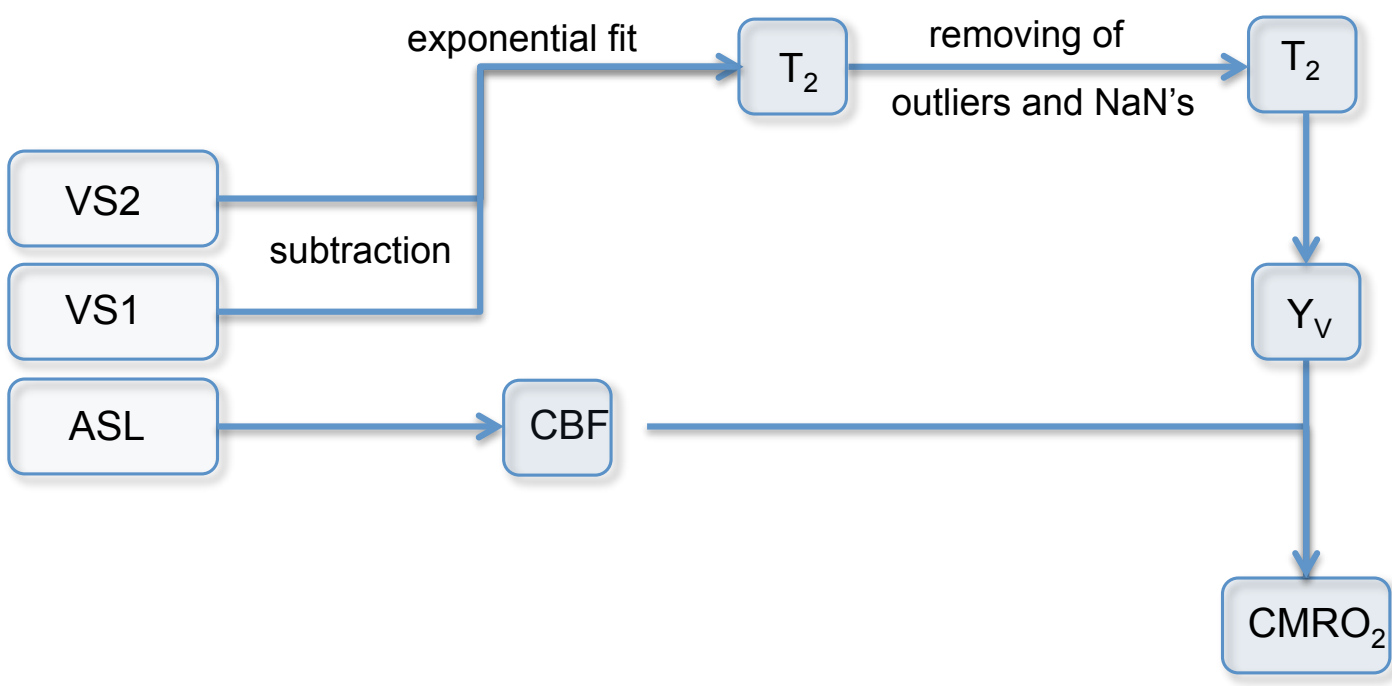

Figure 3.13: Postprocessing steps for QUIXOTIC data. 


\section{Measurement protocol}

Quixotic and turbo QUIXOTIC were used to evaluate $Y_{v}$ and $\mathrm{CMRO}_{2}$ values.

1. Time of flight (TOF) imaging sequence to adjust labelling slab of pCASL

2. $\mathrm{pCASL} T R=3500 \mathrm{~ms} ; \mathrm{TE}=14 \mathrm{~ms} ; 40$ measurements; in-plane resolution $3.6 \mathrm{~mm}$; slice thickness $=10 \mathrm{~mm} ; \mathrm{TA}=2: 24 \mathrm{~min}$

3. Turbo QUIXOTIC with sequence parameters: $T R=4000 \mathrm{~ms} ; \mathrm{TE}=20,40, \ldots, 180,200 \mathrm{~ms}$; 30 averages; in-plane resolution $=3.6 \mathrm{~mm}$; slice thickness $=10 \mathrm{~mm} ; 5 \mathrm{EPI}$ shots; $\mathrm{TA}=5: 59 \mathrm{~min}$ per control $/$ tag; $v_{\text {cutoff }}=2.0 \mathrm{~cm} / \mathrm{s} ; \mathrm{TO} 1=400 \mathrm{~ms} ; \mathrm{TO}=725 \mathrm{~ms}$; partial fourier $=6 / 8$; readout bandwidth $=1628 \mathrm{~Hz} / \mathrm{Px}$

4. "Traditional" Quixotic with identical parameters as turbo QUIXOTIC but instead of 10 TE times, 5 different T2prep durations and thus different TEeff $=28 \mathrm{~ms}, 56 \mathrm{~ms}$, $112 \mathrm{~ms}, 168 \mathrm{~ms}, 224 \mathrm{~ms}$; TA=5:59 min per control/tag and per TEeff 


\section{Chapter 4}

\section{Experiments and Results}

In this chapter the experiments and results which were carried out using methods described in chapter 3 are shown. Discussion and interpretation of these results can be found in chapter 5 .

\subsection{Mapping of Absolute Water Content and Longitudinal Relaxation Times}

\subsubsection{Sequence Parameters}

For each sequence type the same sequence parameters were used in phantom and in vivo:

- TAPIR: $\mathrm{TR}=22 \mathrm{~ms}, \mathrm{TE}=9.74 \mathrm{~ms}, \mathrm{TI}=20 \mathrm{~ms}$, flip angle $(\mathrm{FA})=25^{\circ}$, bandwidth $(B W)=400 \mathrm{~Hz} / \mathrm{Px}$, resolution $1 \mathrm{~mm} \times 1 \mathrm{~mm} \times 2 \mathrm{~mm}$, EPI factor $=5$, GRAPPA factor $=2$, time points $(T P)=20, \tau=2400 \mathrm{~ms}$, slices $=32$, concatenations $=4$, acquisition time $(T A)=6: 44 \mathrm{~min}$

- TAPIR inversion efficiency measurement: same settings as for TAPIR but resolution $3.1 \mathrm{~mm} \times 3.1 \mathrm{~mm} \times 2 \mathrm{~mm}, \mathrm{TA}=0.53 \mathrm{~min}$

- QUTE (slab-selective 3d): first $T E=2.3 \mathrm{~ms}, \triangle T E=2.1 \mathrm{~ms} ; 11$ echoes, $T R=34 \mathrm{~ms}$; $\mathrm{BW}=500 \mathrm{~Hz} / \mathrm{Px}$, resolution $1 \mathrm{~mm} \times 1 \mathrm{~mm} \times 2 \mathrm{~mm}$, GRAPPA factor $=2, F A=50^{\circ}$, $\mathrm{TA}=3: 41 \mathrm{~min}$ 


\subsubsection{Phantom Results}

A so-called "revolver phantom" comprising nine falcon tubes filled with different mixtures of distilled water, $\mathrm{D}_{2} \mathrm{O}$ and $\mathrm{MnSO}_{4}$ was used for phantom experiments. In a first step, the $T_{1}$ relaxation times of all these tubes were determined at $3 \mathrm{~T}$ using a gold standard spectroscopic inversion recovery sequence. The results accessed from the phantom using TAPIR were compard with the spectroscopic values to validate the accuracy of $T_{1}$ and $\mathrm{H}_{2} \mathrm{O}$.

For the quantification of the accuracy, ROls were defined manually for each tube using ImageJ [130]. $T_{1}$ and $\mathrm{H}_{2} \mathrm{O}$ values obtained with TAPIR and with the spectroscopic inversion recovery experiment of each tube are presented in Table 4.1.

\begin{tabular}{ccc||ccc}
$T_{1_{\text {spect. }}}[\mathrm{ms}]$ & $T_{1_{\text {meas. }}}[\mathrm{ms}]$ & Accuracy [\%] & $\mathrm{H}_{2} \mathrm{O}_{\text {real }}[\%]$ & $\mathrm{H}_{2} \mathrm{O}_{\text {meas. }}[\%]$ & Accuracy [\%] \\
\hline 271 & $277 \pm 16$ & 2 & 50 & $49 \pm 2$ & -1 \\
442 & $446 \pm 17$ & 1 & 60 & $57 \pm 2$ & -3 \\
660 & $652 \pm 24$ & -1 & 65 & $67 \pm 2$ & 2 \\
753 & $748 \pm 27$ & -1 & 70 & $70 \pm 2$ & 0 \\
941 & $923 \pm 27$ & -2 & 75 & $73 \pm 2$ & -2 \\
1291 & $1244 \pm 39$ & -4 & 80 & $83 \pm 3$ & 3 \\
1482 & $1458 \pm 72$ & -2 & 85 & $84 \pm 4$ & -1 \\
1993 & $1955 \pm 115$ & -2 & 90 & $91 \pm 5$ & 1 \\
3066 & $3003 \pm 279$ & -2 & 100 & $91 \pm 7$ & -9
\end{tabular}

Table 4.1: Quantitative values and their differences in $\%$ to previously measured gold standard spectroscopic values or to the known concentrations of $\mathrm{H}_{2} \mathrm{O}$ and $T_{1}$ measurements.

\subsubsection{In Vivo Results}

It has been demonstrated several times [104] that the TAPIR method allows for an accurate and robust quantification of the $T_{1}$ values of in vivo the human brain. In vivo measurements were performed on ten healthy volunteers (all right-handed male, mean age of $25 \pm 3$ ) after written consent was obtained according to the ethical guidelines of our institution.

For a representative subject an axial single slice of the whole $\mathrm{H}_{2} \mathrm{O}$ map is presented in Figure 4.1a), whereas the corresponding $T_{1}$ map is depicted in Figure 4.1b). Values of global white and grey matter are obtained using mean values of the corresponding histogram peaks. Representative histograms of a quantitative $\mathrm{H}_{2} \mathrm{O}$ and a $T_{1}$ map are shown in Figure 4.2a) and b). Mean $T_{1}$ and $\mathrm{H}_{2} \mathrm{O}$ content values from white and grey matter regions of all healthy volunteers and average values over all volunteers are presented in Table 4.2. The measured values show good agreement with the $T_{1}$ value ranges found in the literature for white and grey matter (e.g. $\mathrm{H}_{2} \mathrm{O}: \mathrm{WM}: 70 \pm 2 \%$; GM: $83 \pm 3 \%[90,91,131,132] ; T_{1}$ : WM: 
$832 \pm 10 \mathrm{~ms}$ [92], $790 \pm 21 \mathrm{~ms}$ [133], $1110 \pm 40 \mathrm{~ms}$ [134], $1084 \pm 45 \mathrm{~ms}$ [135]; GM: $1331 \pm 13 \mathrm{~ms}$ [92], $1421 \pm 43 \mathrm{~ms}$ [133], $1470 \pm 50 \mathrm{~ms}$ [134], $1820 \pm 114 \mathrm{~ms}$ [135]).

\begin{tabular}{c|cc|cc} 
& \multicolumn{2}{|c|}{$\mathrm{H}_{2} \mathrm{O}[\%]$} & \multicolumn{2}{c}{$T_{1}[\mathrm{~ms}]$} \\
\hline & $\mathrm{WM}$ & $\mathrm{GM}$ & $\mathrm{WM}$ & $\mathrm{GM}$ \\
\hline & 70 & 85 & 875 & 1515 \\
& 72 & 85 & 960 & 1500 \\
& 72 & 86 & 940 & 1501 \\
& 70 & 86 & 891 & 1485 \\
& 72 & 86 & 930 & 1531 \\
& 69 & 82 & 926 & 1495 \\
& 70 & 86 & 927 & 1525 \\
& 71 & 85 & 921 & 1507 \\
& 68 & 83 & 910 & 1519 \\
& 69 & 83 & 904 & 1513 \\
\hline \hline average & $70.3 \pm 1.4$ & $84.7 \pm 1.5$ & $918 \pm 24$ & $1509 \pm 14$
\end{tabular}

Table 4.2: Quantitative values of $\mathrm{H}_{2} \mathrm{O}$ and $T_{1}$ measurements WM and GM utilising TAPIR on ten healthy volunteers.

Figure 4.1: Representative slice of $\mathrm{H}_{2} \mathrm{O}$ and $T_{1}$ maps for a single healthy volunteer measured utilising TAPIR.
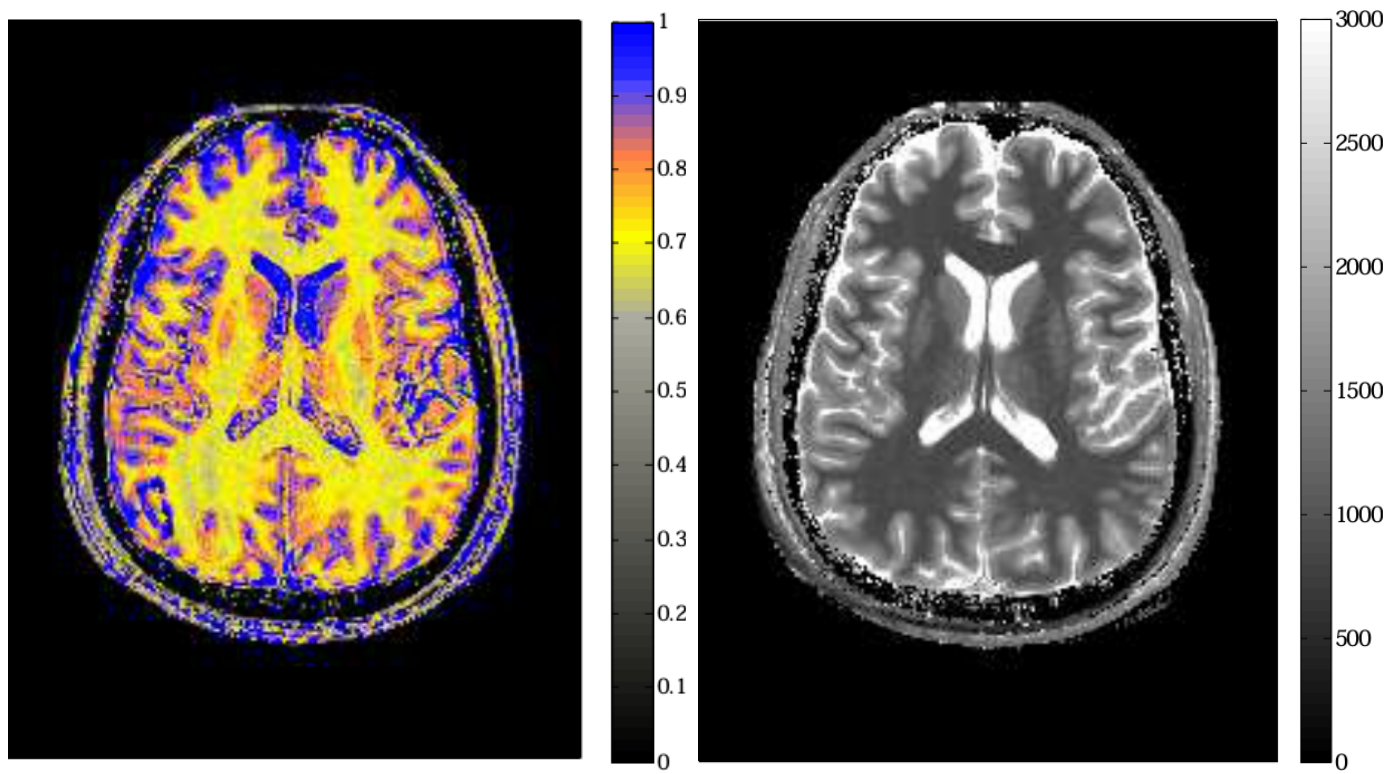

(a) Representative slice of a quantitative $\mathrm{H}_{2} \mathrm{O}$ map. (b) Representative slice of a quantitative $T_{1}$ map. Values are given in \%.

Values are given in ms. 
Figure 4.2: Histograms of $\mathrm{H}_{2} \mathrm{O}$ and $T_{1}$ maps for a healthy volunteer measured utilising TAPIR.
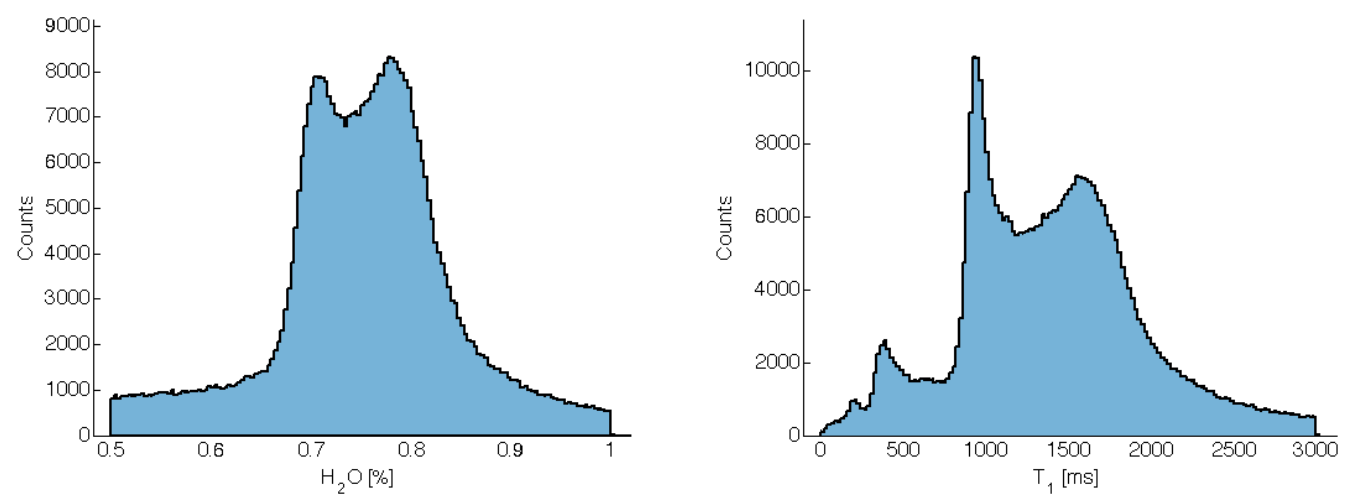

(a) Histogram of a quantitative $\mathrm{H}_{2} \mathrm{O}$ map. Values (b) Histogram of a quantitative $T_{1}$ map. Values are are given in $\%$. given in $\mathrm{ms}$. 


\subsubsection{Discussion and Conclusions}

In general acquisition times of $\mathrm{H}_{2} \mathrm{O}$ quantification, especially with the use of the originally published TAPIR sequence, are too long for clinical acceptance. With the enhanced TAPIR sequence including adiabatic inversion pulses, echo time shifting and last but not least parallel imaging techniques, the acquisition times can be further reduced. The results presented in this chapter show that the accuracy (below $\pm 4 \%$ for $T_{1}$ and below $\pm 3 \%$ for clinically relevant $\mathrm{H}_{2} \mathrm{O}$ values in phantom experiments) and precision (below $\pm 5 \%$ for clinically relevant $T_{1}$ and below $\pm 5 \%$ for clinical relevant $\mathrm{H}_{2} \mathrm{O}$ values in phantom experiments) of the accelerated versions are shown to be good.

It has been shown that with a given set of parameters it is possible to acquire accurate $T_{1}$ and $\mathrm{H}_{2} \mathrm{O}$ maps in clinically acceptable measurement times. This was achieved using the TAPIR sequence combined with a fast and accurate $T_{2}^{*}$ mapping sequence (QUTE). The use of cerebrospinal fluid as a reference value for water content makes the use of external reference probes obsolete. At higher field strenghts it is not possible to use reference probes for calibration to a known $\mathrm{H}_{2} \mathrm{O}$ content because it is not possible to exactly compensate the receive profile outside the brain and thus using the cerebrospinal fluid as a reference value was proposed by $[10,11]$.

It has also been demonstrated in phantom experiments that the presented method shows good agreement between the measured and the gold standard spectroscopic $T_{1}$ values as well as between the measured and known water contents. The method for quantification of $T_{1}$ relaxation time and $\mathrm{H}_{2} \mathrm{O}$ content mapping was performed on a cohort of ten healthy volunteers. Results of the study are in line with the known literature values for white $\left(T_{1}=918 \pm 24 \mathrm{~ms}, \mathrm{H}_{2} \mathrm{O}=70.3 \pm 1.4 \%\right)$ and grey matter $\left(T_{1}=1509 \pm 14 \mathrm{~ms}\right.$, $\left.\mathrm{H}_{2} \mathrm{O}=84.7 \pm 1.5 \%\right)$.

Taking the total acquisition time of 11:18 minutes into account, the presented method paves the way to a clinical appliance of $T_{1}$ and $\mathrm{H}_{2} \mathrm{O}$ mapping of the human brain. For later use in ${ }^{17} \mathrm{O} \mathrm{MRI}$ low-resolution images would be sufficient and thus the acquisition time can be reduced drastically. 


\subsection{Natural Abundance ${ }^{17} \mathrm{O} \mathrm{MRI}$}

\subsubsection{Natural Abundance ${ }^{17} \mathrm{O} \mathrm{MRI}$ in vivo}

Natural abundance images of a healthy male volunteer were acquired in vivo after having gained written consent within a clinical trial on a 9.4 T MRI system (Siemens AG, Erlangen, Germany). A custom-built circularly polarized birdcage coil tuned to the ${ }^{17} \mathrm{O}$ Larmor frequency $(54.2 \mathrm{MHz}$ at $9.4 \mathrm{~T}$ ) with an inner diameter of $280 \mathrm{~mm}$ and a length of $240 \mathrm{~mm}$ was used for transmission and reception (Figure 4.3). A twisted projection imaging sequence (TPI) [70] was used for these measurements.

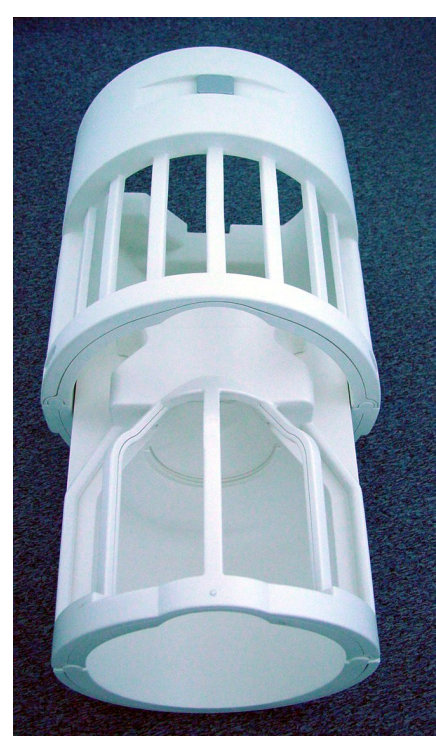

Figure 4.3: $\mathrm{Tx} / \mathrm{Rx}$ coil for ${ }^{17} \mathrm{O}$ at $9.4 \mathrm{~T}$.
The sequence parameters were: $\mathrm{TE}=0.4 \mathrm{~ms}, \mathrm{TR}=11.4 \mathrm{~ms}$, readout time $=5.12 \mathrm{~ms}$. The maximum specific absorption rates (SAR) limit imposed the use of a flip angle of 30 degrees. With these sequence parameters two sets of data were acquired. The first data set had a nominal resolution of $5 \mathrm{~mm}$ isotropic and was obtained with 10 averages in 11:19 min. The second data set had a nominal resolution of $10 \mathrm{~mm}$ isotropic and was obtained with 8 averages in 8:08 $\mathrm{min}$. The raw data was regridded to a Cartesian grid using a standard gridding algorithm written by Zwart and Pipe which is freely available [136]. To improve the SNR, a Blackman filter [137] was applied to $k$-space data of the $5 \mathrm{~mm}$ measurements. Representative slices of each measurement can be found in Figure 4.4 . 


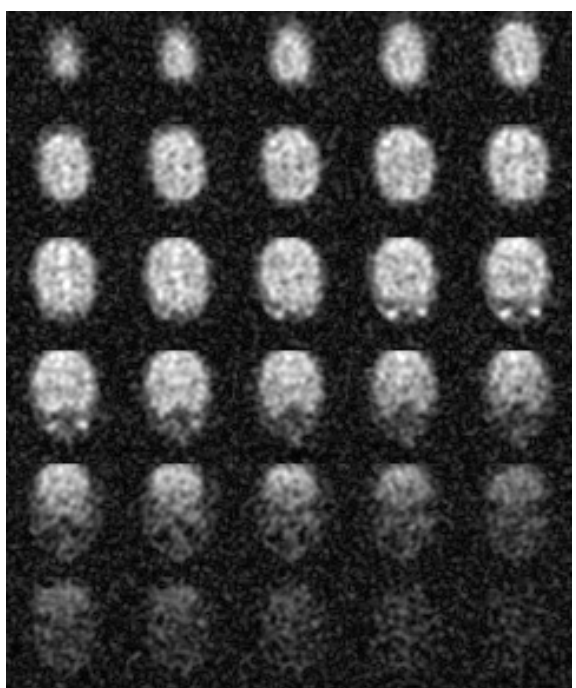

(a) $5 \mathrm{~mm}$ isotropic ${ }^{17} \mathrm{O}$ image

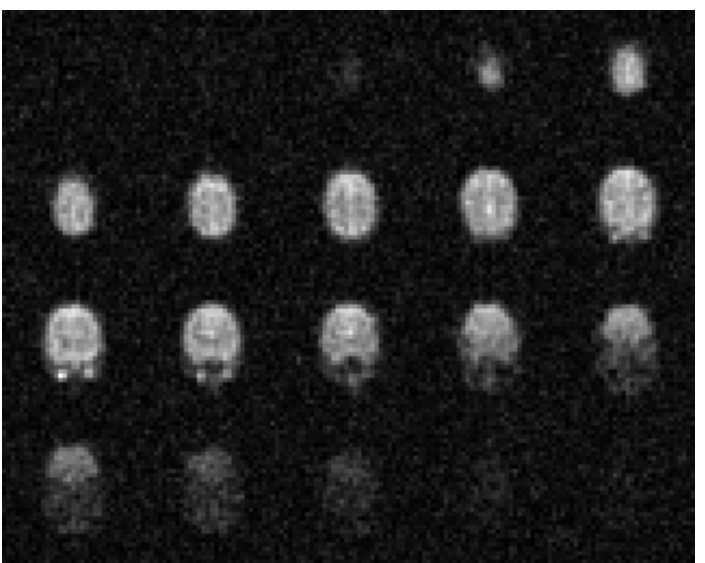

(b) $10 \mathrm{~mm}$ isotropic ${ }^{17} \mathrm{O}$ image

Figure 4.4: Natural abundance ${ }^{17} \mathrm{O}$ images of a healthy volunteer acquired at $9.4 \mathrm{~T}$ with nominal isotropic resolutions of $5 \mathrm{~mm}$ and $10 \mathrm{~mm}$ in $11: 19 \mathrm{~min}$ and $8: 08 \mathrm{~min}$ respectively [2,3], displayed is the transverse view. 


\subsubsection{Natural Abundance ${ }^{17} \mathrm{O} \mathrm{MRI}$ in phantom}

Following natural abundance MR experiments were carried out on a home-integrated preclinical 9.4 T MRI scanner. This system, shown in Figure 4.5, consists of an actively-shielded horizontal bore superconducting magnet and gradient/shim coil with an inner diameter of $120 \mathrm{~mm}$ with a gradient strength up to $400 \mathrm{mT} / \mathrm{m}$ and up to 2 nd order shim. The main components are made by Varian/Agilent Technologies, USA, and major system electronics and clinical software/user interface (SYNGO) from Siemens Healthcare, Erlangen, Germany. This allows transferring all MR pulse sequences directly from the human Siemens clinical MRI system and vice versa.

Two identical circularly-polarised tuneable high-pass birdcage coils were designed and constructed using a flexible printed circuit board [138]. These coils were self-shielded by the use of copper tapes overlapped alternatively. Two types of home-built Transmit/Receive (T/R) switches controlled by PIN diodes were designed and embedded into the coil set. For ${ }^{17} \mathrm{O}$ the frequency a hybrid coupler was used while for ${ }^{1} \mathrm{H}$, two Wilkinson's power splitters were utilised in order to divide the transmit power and to combine receive signals. All preamplifiers were protected by a couple of phase shifters $(\pi)$ either made by lumped elements or adjusted by the coaxial cable length. Two $1 \mathrm{~kW}$ RF amplifiers (Barthel, Germany) were utilised - one narrowband $(370-400 \mathrm{MHz})$ for ${ }^{1} \mathrm{H}$ and one broadband $(50-180 \mathrm{MHz})$ for ${ }^{17} \mathrm{O}$.

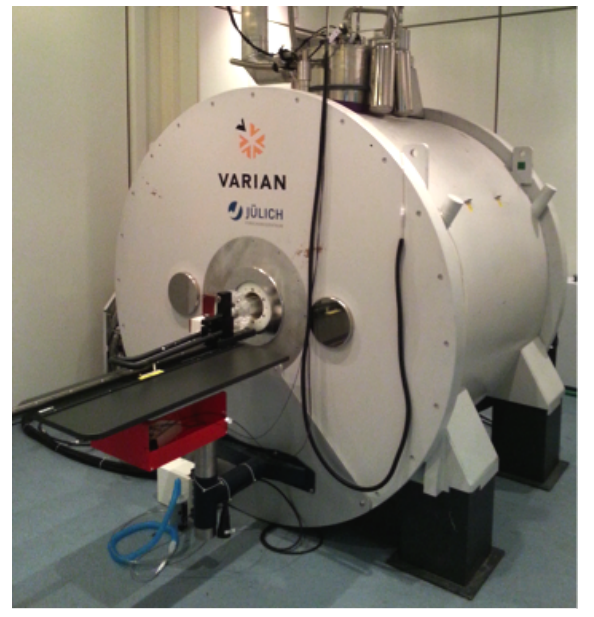

Figure 4.5: $9.4 \mathrm{~T}$ home-integrated preclinical system.

Figure 4.6 shows the same axial view of a phantom comprising of seven tubes filled with distilled water. First measured at the proton frequency with a 3d Cartesian gradient echo sequence and a TPI sequence with the same nominal isotropic resolution of $1 \mathrm{~mm}$ at comparable measurement times of 3:12 min and 3:20 min respectively.

Following these measurements the coil was changed to ${ }^{17} \mathrm{O}$ from the back of the scanner without moving the phantom and a set of measurements was carried out. First a 3d Cartesian gradient echo imaging sequence with a nominal isotropic resolution of $1.2 \mathrm{~mm}$ in an acquisition time of $25 \mathrm{~min}$ and secondly a TPI sequence [70] with a comparable resolution of $1.3 \mathrm{~mm}$ in $20 \mathrm{~min}$ were performed. Following this, lower resolution acquisitions were performed with $2 \mathrm{~mm}$ and $3 \mathrm{~mm}$ nominal isotropic resolution in acquisition times of $5 \mathrm{~min}$ and $1 \mathrm{~min}$ respectively. 


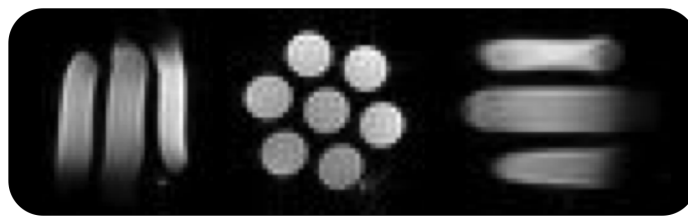

(a) $1 \mathrm{~mm}$ isotropic ${ }^{1} \mathrm{H}$ GRE image; Acquisition Time $(\mathrm{TA})=3: 12 \mathrm{~min}$

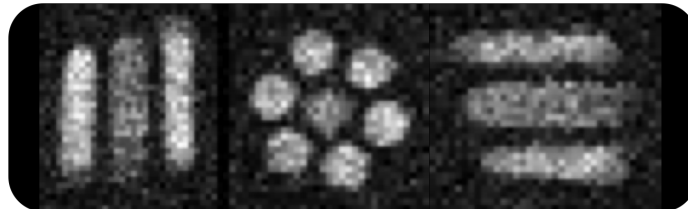

(c) $1.2 \mathrm{~mm}$ isotropic ${ }^{17} \mathrm{O}$ GRE image; TA $=25 \mathrm{~min}$

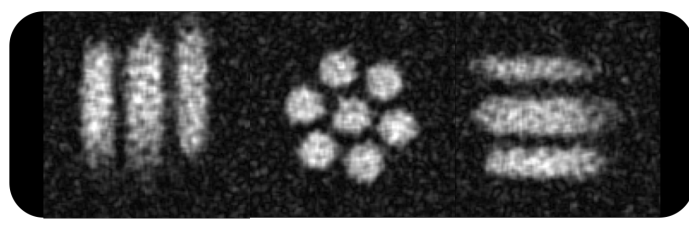

(e) $2 \mathrm{~mm}$ isotropic ${ }^{17} \mathrm{O} \mathrm{TPI}$ image; TA $=5 \mathrm{~min}$

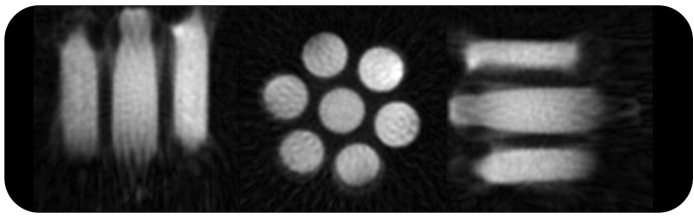

(b) $1 \mathrm{~mm}$ isotropic ${ }^{1} \mathrm{H}$ TPI image; TA $=3: 20 \mathrm{~min}$

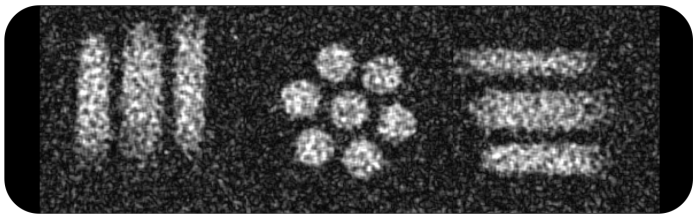

(d) $1.3 \mathrm{~mm}$ isotropic ${ }^{17} \mathrm{O} \mathrm{TPI}$ image; TA=20 $\mathrm{min}$

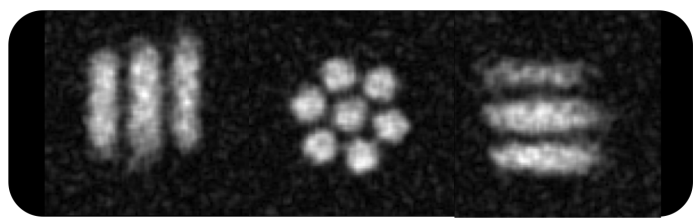

(f) $3 \mathrm{~mm}$ isotropic ${ }^{17} \mathrm{O} \mathrm{TPI}$ image; TA $=1 \mathrm{~min}$

Figure 4.6: Three axial views of a phantom comprising seven tubes of distilled water measured at a 9.4 T small animal MRI scanner. First two acquisitions show the ${ }^{1} \mathrm{H}$ and the last four measurements the ${ }^{17} \mathrm{O}$ signal.

All relevant sequence parameters can be found in Table 4.3. Details about TPI sequence can be found in section 3.4.1 and in the literature [70].

\begin{tabular}{l||c|c|c|c|c|c}
\multicolumn{1}{c||}{} & \multicolumn{2}{c|}{${ }^{1} \mathrm{H}$} & \multicolumn{4}{c}{17} \\
\hline & GRE & TPI & GRE & TPI & TPI & TPI \\
\hline resolution [mm] & 1 & 1 & 1.2 & 1.3 & 2 & 3 \\
TE [ms] & 2.54 & 0.55 & 2.74 & 0.1 & 0.1 & 0.1 \\
TR [ms] & 100 & 100 & 20 & 20 & 20 & 20 \\
averages & 1 & 1 & 64 & 20 & 5 & 1 \\
projections & - & 2000 & - & 3000 & 3000 & 3000 \\
$\mathrm{p}[\%]$ & - & 80 & - & 100 & 100 & 100 \\
\hline TA [min] & $3: 12$ & $3: 20$ & 25 & 20 & 5 & 1
\end{tabular}

Table 4.3: Sequence parameters which were used for natural abundance imaging on a $9.4 \mathrm{~T}$ preclinical MRI system. 


\subsubsection{Discussion and Conclusions}

In all in vivo images acquired at the 9.4 T whole-body human MRI scanner the eyes and the two hemispheres of the human brain are clearly visible. Within the images it is possible to distinguish between white and grey matter, especially in images with sufficient SNR such as in the low resolution acquisition in Figure 4.4b), keeping in mind that these images where acquired at natural abundance of only $0.037 \%$. In further studies the sequence parameters could be optimised to increase the nominal FA which inherently increases the SNR.

Further sequence parameter optimisation was performed for the phantom images which were acquired at the 9.4 T small animal scanner. Even in the shortest acquisition time of $1 \mathrm{~min}$ with a nominal resolution of $3 \mathrm{~mm}$ all phantom tubes are clearly distinguishable. The dataset, which was acquired with a cartesian gradient echo sequence, shows significant blurring due to relatively long readout and echo times.

Further studies on the human scanner were not continued, since the magnet had major technical issues after a quench and is still in repair. 


\subsection{Quantification of $\mathrm{CMRO}_{2}$}

\subsection{1 $T_{2}$ Measurements}

Despite of the use of the VS module, the same measurement protocol as used for in vivo measurements to quantify $\mathrm{CMRO}_{2}$ with QUIXOTIC (see section 3.4.2.3.2) was employed for $T_{2}$ quantification in phantoms, in order to prove accuracy of the method with respect to relaxation time mapping.

Calculated $\mathrm{T}_{2}$ values can be found in Table 4.4 along with the corresponding gold standard spectroscopically measured values of a resulting in $T_{2}$ within a physiological range. The calculated values are in good agreement with the spectroscopic results.

\begin{tabular}{c|c|c}
$\mathrm{T}_{2_{\text {spectroscopic }}}[\mathrm{ms}]$ & Multi-echo spin echo $\mathrm{T}_{2}[\mathrm{~ms}]$ & Difference [\%] \\
\hline 32 & $33 \pm 4$ & 3 \\
52 & $54 \pm 5$ & 4 \\
62 & $63 \pm 5$ & 2 \\
79 & $77 \pm 7$ & -2
\end{tabular}

Table 4.4: Quantitative values of $T_{2}$ measured in four different Falcon tubes with different $T_{2}$ values in a physiological range and comparison with previously measured gold standard spectroscopic values.

\subsubsection{QUIXOTIC}

Figure 4.7 shows representative $\mathrm{Y}_{v}$ and $\mathrm{CMRO}_{2}$ maps of a healthy male volunteer measured with

a) standard QUIXOTIC sequence utilizing a T2prep magnetisation preparation module and

b) segmented multi-shot multi echo spin echo sequence after data processing by applying the QUIXOTIC theory on a voxel-by-voxel basis

$\mathrm{Y}_{v}$ is relatively uniform across the whole imaged slice, while $\mathrm{CMRO}_{2}$ values are different in grey matter (GM) and white matter (WM), which has been shown by Bolar et al. [14]. This is further known from ${ }^{17} \mathrm{O}$ MRI $[61,75,82]$ and ${ }^{15} \mathrm{O}$ PET [139] experiments as well. Labelling parameters were kept the same and chosen to be identical to Bolar et al. [14]. Quantitative values for $\mathrm{CBF}, \mathrm{CMRO}_{2}, \mathrm{~T}_{2}$ and $\mathrm{Y}_{v}$ in grey matter in four healthy volunteers are shown in Table 4.5 .

The mean values in GM of all healthy volunteers are found to be $Y_{v}=0.61 \pm 0.03$, $\mathrm{T}_{2}=54 \pm 4 \mathrm{~ms}, \mathrm{CMRO}_{2}=174 \pm 13 \mu \mathrm{mol} / 100 \mathrm{~g} \mathrm{~min}$ and $\mathrm{CBF}=53 \pm 3 \mathrm{ml} / 100 \mathrm{~g} \mathrm{~min}$. The results show good agreement between each other and further agree with literature $\mathrm{CMRO}_{2}$ values 
in GM, especially to gold standard ${ }^{15}$ O PET based measurements (Bolar (QUIXOTIC) [14]: $\mathrm{CMRO}_{2}=125 \pm 15 \mu \mathrm{mol} / 100 \mathrm{~g}$ min, Leenders ( ${ }^{15} \mathrm{O}$ PET) [139]: $\mathrm{CMRO}_{2}=186 \pm 19 \mu \mathrm{mol} / 100 \mathrm{~g}$ min). $\mathrm{CMRO}_{2}$ of white matter regions are reported to be significantly lower as reported by different studies (e.g. $\mathrm{CMRO}_{2 \mathrm{WM}}=71 \pm 4 \mu \mathrm{mol} / 100 \mathrm{~g} \min$ [139]) which explains the slightly lower $\mathrm{CMRO}_{2}$ values measured by means of QUIXOTIC by Bolar et. al [14] caused by partial volume effects due to lower imaging resolution and thus contamination of the GM values with lower WM values.

Subject 4 is measured twice first (named subject $4 a$ ) with the new established multishot multi echo turbo QUIXOTIC utilising HSn pulses and secondly (named subject 4b) with the standard QUIXOTIC approach utilising a T2prep as published by Bolar [14]. Especially the agreement of the results utilising a T2prep module and the multi-shot capabilities in turbo QUIXOTIC is emphasised.

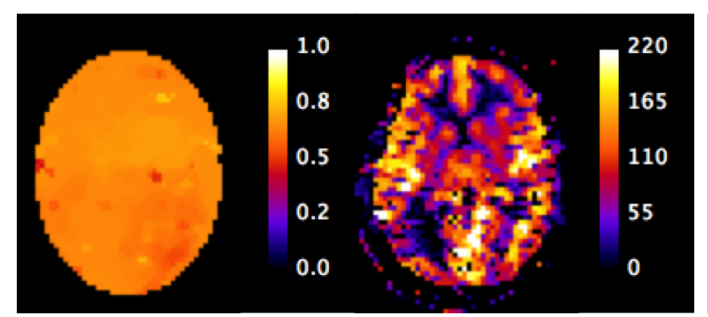

(a) Standard QUIXOTIC utilising a T2prep module

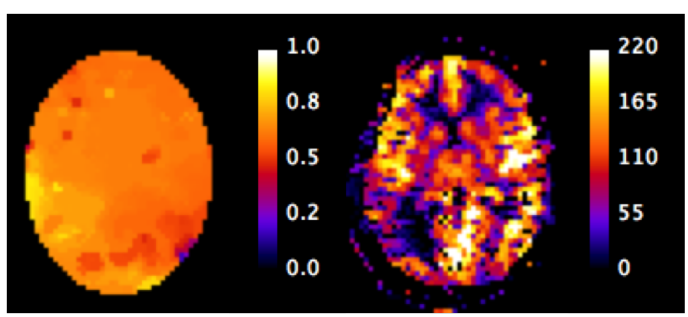

(b) Turbo QUIXOTIC utilising a multishot multi echo spin echo with adiabatic $\mathrm{HSn}$ pulses used for refocusing

Figure 4.7: $Y_{v}$ (left) and $\mathrm{CMRO}_{2}\left[\frac{\mu \mathrm{mol}}{100 g \mathrm{~min}}\right]$ (right) of a representative subject

\begin{tabular}{l|c|c|c|c} 
Measurement & $\mathrm{CBF}\left[\frac{\mu \mathrm{l}}{100 \mathrm{gmin}}\right]$ & $\mathrm{CMRO}_{2}\left[\frac{\mu \mathrm{mol}}{100 \mathrm{gmin}}\right]$ & $T_{2}[\mathrm{~ms}]$ & $Y_{v}$ \\
\hline 1 & $50 \pm 7$ & $187 \pm 59$ & $48 \pm 11$ & $0.56 \pm 0.10$ \\
2 & $57 \pm 11$ & $188 \pm 59$ & $53 \pm 11$ & $0.61 \pm 0.09$ \\
3 & $55 \pm 10$ & $170 \pm 42$ & $57 \pm 11$ & $0.63 \pm 0.06$ \\
4a & $52 \pm 9$ & $166 \pm 36$ & $55 \pm 11$ & $0.62 \pm 0.05$ \\
4b & $53 \pm 9$ & $159 \pm 31$ & $57 \pm 5$ & $0.64 \pm 0.03$
\end{tabular}

Table 4.5: $\mathrm{CBF}, \mathrm{CMRO}_{2}, T_{2}$ and $Y_{v}$ results in four healthy volunteers. One of the volunteers was measured twice, once with the multishot turbo QUIXOTIC variant (a) and once with the original published method utilising a T2prep with different $T_{2}$ weightings (b). 


\subsubsection{Discussion and Conclusions}

With the presented enhancements in the turbo QUIXOTIC approach, a quantitative assessment of clinically relevant parameters such as $\mathrm{CMRO}_{2}$ is possible in clinically acceptable measurement times. This paves the way to its applications in research and clinical trials. The agreement between the originally published and the enhanced method as well as the agreement with available literature values is good. Especially the agreement to gold standard ${ }^{15} \mathrm{O}$ PET based measurements is highlighted. The slight difference to the lower QUIXOTIC values published by Bolar et. al [14] may be caused by lower imaging resolution and thus contamination of the GM values by partial volume effects of smaller $\mathrm{WM} \mathrm{CMRO}_{2}$ values. 


\section{Chapter 5}

\section{Discussion, Conclusions and Outlook}

The main goal of this thesis was to achieve non-invasive measurements of $\mathrm{CMRO}_{2}$ in healthy volunteers based on either ${ }^{17} \mathrm{O}$ or on ${ }^{1} \mathrm{H}$ MR imaging. Summarising the desired methods, which were presented in the introduction of this thesis in chapter 1 , two methods of choice exist. The first method based on direct detection of the ${ }^{17} \mathrm{O}$ at ultra-high magnetic field needs accurate and quantitative in vivo $\mathrm{H}_{2} \mathrm{O}$ maps as a basis for $\mathrm{CMRO}_{2}$ calculations (see section 3.1.3).

This was achieved here by an extended implementation of the TAPIR sequence for quantitative in vivo $T_{1}$ relaxation time measurements and $\mathrm{H}_{2} \mathrm{O}$ quantification, as described in section 3.3.3. The results presented in chapter 4 demonstrate that it is possible to acquire high-resolution $T_{1}$ and $\mathrm{H}_{2} \mathrm{O}$ maps with volume coverage in a clinically acceptable measurement times of 11:18 min in total. The in vivo precision of the mean values and the accuracy, referenced to the gold standard spectroscopic inversion recovery experiment of WM and GM are approximately $2 \%$ and $1 \%$, respectively. This method can be used on its own for studies which include relaxation time mapping or a quantitative measurement of water content and in studies where ${ }^{17} \mathrm{O}$ imaging is used.

Imaging natural abundance concentrations of ${ }^{17} \mathrm{O}$ is the basis for accurate $\mathrm{CMRO}_{2}$ quantification based on the ${ }^{17} \mathrm{O}$ signal. These measurements were carried out with the methods described in section 3.2. Initial measurements with an RF coil developed in-house were performed in vivo and in a phantom. The results in section 4.2 .1 show the feasibility of acquiring natural abundance images with a nominal resolution of $5 \mathrm{~mm}$ or $10 \mathrm{~mm}$ of the directly measured ${ }^{17} \mathrm{O}$ signal in vivo at a $9.4 \mathrm{~T}$ scanner with sufficient SNR in acceptable measurement times of 11:19 min and 8:08 min respectively. Phantom measurements of the natural abundance signal were performed additionally on a small animal $9.4 \mathrm{~T}$ generating a basis for further studies. With smaller imaging coils, which are used on small animal scanner systems, images with sufficient SNR can be acquired on a nominal resolution of $3 \mathrm{~mm}$ in $1 \mathrm{~min}$ acquisition times. Further in vivo studies on a whole-body $9.4 \mathrm{~T}$ human scanner with improved SNR due to sequence parameter optimisation pave the way to $\mathrm{CMRO}_{2}$ quanti- 
fication by means of ${ }^{17} \mathrm{O}$ imaging and will be carried out in future studies. These studies will require the knowledge of quantitative $\mathrm{H}_{2} \mathrm{O}$ contents in the brain and thus quantitative estimation of absolute $\mathrm{H}_{2} \mathrm{O}$ and ${ }^{17} \mathrm{O}$ imaging methods are linked.

The second method of choice to quantify $\mathrm{CMRO}_{2}$ in vivo is, as already mentioned in the introduction of this thesis in chapter 1 , based on quantification of transverse relaxation rates, $T_{2}$, of venous blood which is linked via Fick's principle [1] to its oxygenation. In section 4.3 a further development of a $\mathrm{CMRO}_{2}$ mapping sequence based on this principle is presented. Using a multi-echo, spin-echo imaging approach the measurement is accelerated compared to the acquisition of different $T_{2}$ weightings using a T2prep module as in the originally published imaging sequence. The use of a gradient spoiling scheme and the application of adiabatic refocusing pulses (described in more detail in chapter 3 ) prevents stimulated echoes leading to more accurate $T_{2}$ values. Otherwise these would influence $T_{2}$ quantification and thus result in further errors in $Y_{v}$ and $\mathrm{CMRO}_{2}$. The use of advanced adiabatic HSn pulses, as introduced by Tannus [28] and described in detail in section 2.3.6, leads to lower SAR levels and thus enables longer echo trains. These methods were implemented and tested for phantom and in vivo experiments. A comparison with gold standard spectroscopically measured $T_{2}$ values and to the original published method was performed. Phantom results show an accuracy of below $3 \%$ for $T_{2}$ values in a physiological range. The $\mathrm{CMRO}_{2}, T_{2}$ and $Y_{v}$ values in in vivo measurements are in a good agreement with literature values and with the original published method.

Functional activation alters the ratio of $\mathrm{CMRO}_{2}$ to the cerebral metabolic rate of glucose consumption (CMR-Glc) which implies an anaerobic metabolic process [140] and is shown to be changed in patients with cerebral ischemia [141]. Simultaneously performed quantitative measurements of $\mathrm{CMRO} 2$ and $\mathrm{CMR}-\mathrm{Glc}$ were not possible by means of PET because of the indistinguishability of positrons emitted by ${ }^{15} \mathrm{O}$ - (used in $\mathrm{CMRO}_{2}$ quantification) and ${ }^{19} \mathrm{~F}$ - (used in CMR-Glc quantification) labelled PET tracers. The feasibility of quantitative $\mathrm{CMRO}_{2}$ evaluation by $\mathrm{MRI}$ enables future studies and measurements of $\mathrm{CMRO}_{2}$. These will be conducted on the basis of ${ }^{17} \mathrm{O}$ and ${ }^{1} \mathrm{H}$ imaging at $9.4 \mathrm{~T}$ and $3 \mathrm{~T}$ with simultaneously measured FDG-PET to achieve a simultaneous quantitative comparison of $\mathrm{CMRO}_{2}$ and $\mathrm{CMR}$-Glu to further investigate these changes in patients with mild cognitive impairments. 


\section{Chapter 6}

\section{Acknowlegdements}

This thesis would not have been possible without the help and support of many people whom I want to thank.

- Prof. Dr. Jon Shah, the head of the institute and my supervisor, for providing me with the opportunity to work in such an exciting field of research and for giving me useful hints and, whenever needed, having an open door for me.

- Dr. Christophe Phillips and Dr. Evelyne Balteau, my co-supervisors of this thesis, for all the time they spend and your support during the last years.

- Prof. Dr. Walter Backes, Prof. Dr. Ralf Deichmann, Prof. Dr. Michael Kraft and Prof. Dr. Armin Nagel who took the time and effort to judge my work.

- Prof. Dr. Peter de Weerd for all help and endurance in answering my questions and for organisation of such great courses in the framework of the Marie Curie ITN NeuroPhysics \& NWO M-Bic Graduate School

- Kathrin, my beloved wife, who accompanied me during the last years and enriched my life in all parts. Thank you so much for the unquestioned support in all matters.

- My family for their loving support during my entire life and their belief in my abilities.

- All my fellow students and friends for helping me, giving me scientific hints or cheerful coffee breaks. I can't name all and may have forgotten some but respectively the most important in alphabetic order: Chang-Hoon Choi, Christoph Weirich, Daniel Brenner, Daniel Fiege, Desmond Tse, Ezequiel Farrher, Fabian Keil, Ivan Maximov, Johannes Lindemeyer, Jörg Felder, Markus Zimmermann, Melissa Schall, Michael Schwerter, Michaela Gaens, Nuno da Silva, Robert Claeser, Rüdiger Stirnberg, Tony Stöcker, Wieland Worthoff, Zaheer Abbas. 


\section{Appendix A}

\section{Curriculum Vitae}

- Birthdate: $8^{\text {th }}$ of July 1983 in Mülheim an der Ruhr, Germany

- 1994 - 2003: Preuniversity: Karl-Ziegler-Schule Mülheim an der Ruhr, Germany

- 2004 - 2010: Technical University Dortmund, Germany: Diplom Physik

- 2010 - 2013: Maastricht University

- 2013 - today: Research Assistant, Research Centre Jülich, Germany 


\section{Appendix B}

\section{Valorisation}

Researchers at Maastricht University are expected to discuss the social and economic value of the knowledge developed during their doctoral studies. This chapter addresses the current and potential impact of this thesis.

\section{B.1 Social and Economic Relevance}

In this work an enhanced method for quantitative and non-invasive measurements of the $\mathrm{CMRO}_{2}$ in healthy volunteers is presented. Aging and, more importantly, healthy aging is becoming a focus of clinical research since the life expectancies are on the rise. In prosperous countries, Alzheimer's disease is one of the most prevalent neurodegenerative diseases. Novel methods for identifying disease onsets and tracking severity are highly desired for preclinical and clinical testing of disease-modifying therapies. It has been shown that neurodegenerative and metabolic diseases associated with aging and pathologies such as Alzheimer's disease indicate altered values for $\mathrm{CMRO}_{2}$ [71-73] even before anatomical changes are detectable; this makes $\mathrm{CMRO}_{2}$ a perfect molecular imaging candidate for tracking the disease and its severity, and perhaps its early identification.

In the past, $\mathrm{CMRO}_{2}$ was measured by means of radioactive and invasive PET measurements and thus these are not practical on large cohorts of healthy or apparently healthy volunteers. New non-invasive methods such as those presented in section 3.1 pave the way for easier patient handling and clinical acceptance, this is of large impact for social relevance. Shorter measurement times are of economic relevance since the costs of MRI measurement time are a crucial factor in clinical applications. 
Quantitative imaging methods such as relaxation time mapping or quantification of absolute water content are of great interest in clinical applications since standard clinical MR images cannot be directly compared. Most methods for imaging anatomical details in high resolution are relaxation-time weighted, $\mathrm{T}_{1}$-weighted for example, and the results differ for the same patient at different field strengths or when acquired with MRI machines from different vendors or in longitudinal studies. Thus, a quantitative long-term evaluation of patient treatment is not easily feasible. Utilising the quantitative imaging methods, which are shown in this work in section 3.3 , it is possible to acquire quantitative relaxation times, on a voxelwise basis, of the longitudinal relaxation time, $\mathrm{T}_{1}$, and the absolute water content within clinically acceptable measurement times of $11: 18 \mathrm{~min}$ in total. The in vivo precision of the mean values and the accuracy, which is referenced to the gold standard spectroscopic inversion recovery experiment of WM and GM are approximately $2 \%$ and $1 \%$, respectively. 


\section{B.2 Target Group}

This work is of interest not only for academic and basic researchers but also for medical personnel and financial decision makers in clinics. First it enables an easier patient handling compared to ${ }^{15} \mathrm{O}$-based radioactive PET measurements, which decreases corrupted measurement data due to e.g. motion. Second, measurement time and thus running costs of MRI and PET machines can be reduced.

Quantitative imaging techniques are already used by various research sites potentially leading to a better understanding of different diseases and their treatments.

\section{B.3 Products and Services}

The presented quantitative imaging methods and the developments of TAPIR, shown in section 3.3.2.2, already led to various partnerships in research. For example, the developments of this work and a software package for evaluation were used for permeability measurements of the blood brain barrier [142]. Further studies could pave the way for quantitative imaging methods in clinical environments to support diagnostic evaluation processes.

Accessing $\mathrm{CMRO}_{2}$ by non-invasive imaging methods or combining it with simultaneous measurements of other relevant molecular parameters could lead to a deeper knowledge and better understanding of various diseases and their possible treatments. Since ultra-high magnetic field strength MRI is a growing field of current research and the first clinical $7 \mathrm{~T}$ machines are about to be introduced, studies of other nuclei than ${ }^{1} \mathrm{H}$ approaches clinical practice and thus, ${ }^{17} \mathrm{O}$-based measurements become more attractive. ${ }^{1} \mathrm{H}$-based measurements of $\mathrm{CMRO}_{2}$, as they are presented in this work, are the method of choice, whenever ultra-high magnetic field strength is not available. The developments presented in this work have the potential to lead to clinical utility. 


\section{B.4 Innovation}

As Albert Einstein already mentioned

"Life is like riding a bicycle. To keep your balance, you must keep moving."

which implies a continous and ongoing innovation process in life and absolutely in research. Innovative research is of fundamental importance. The work presented in this thesis is yet another step that enhances existing methods.

Ultra-high magnetic field strength MRI with $7 \mathrm{~T}$ and above is a growing topic in research and this is also the case for higher field strength such as $9.4 \mathrm{~T}$, as used in this.

The measurements presented in section 4.1.2 for quantifying absolute water content in the living human brain use state-of-the-art methodologies including adiabatic inversion radiofrequency pulses, parallel imaging techniques, and multi-channel receive RF coils to overcome inhomogeneity effects in $B_{1}^{+}$fields which are a negative sideffect caused by high magnetic field strengths. The method for correcting the $B_{1}^{-}$ receive field inhomogeneity, described briefly in section 3.3.3.3, was developed in our group and is completely new and innovative. Prior to the availability of that method either corrections based on the reciprocity theorem, which does not hold for high field strength, or just simple bias field corrections by homogenisation of the resulted quantitative measurement were used.

The essential in vivo and phantom measurements of the natural abundance of ${ }^{17} \mathrm{O}$ signal at ultra-high field strength (9.4T) presented in section 4.2.1 and thereafter are one of the first presented at that high field strength using a state-of-the-art prototype UHF scanner.

The use of SAR-reduced adiabatic pulses in a T2prep module is an innovative method. Previously, mostly normal adiabatic pulses, which have the negative side-effect of high SAR values or non-adiabatic pulses which in turn have a poor $B_{1}^{+} \mathrm{RF}$ profile were used. 


\section{B.5 Shedule and Implementation}

First, all necessary imaging sequences were implemented for Siemens MRI scanner systems (Siemens AG, Erlangen, Germany) and tested extensively. The good agreement of TAPIR $T_{1}$ measurement results with spectroscopically measured ones triggered great interest in the community, leading to international cooperations with various partners who are using the sequence (e.g. University of New Mexico, Albuquerque, NM; University of Cambridge, UK) or plan future usage (e.g. University of Hawaii, HI).

Second, not only did sequence implementation lead to cooperations but also the evaluation software package in which all used fitting routines and all described $B_{1}^{-}$ corrections were implemented. The software package is called "Juqebox" and is freely available upon request from our research group.

Evaluation of $\mathrm{CMRO}_{2}$ based on ${ }^{17} \mathrm{O}$ imaging data is complex and patient comfort is reduced for this imaging modality. Firstly by special safety issues due to ultra-high field MRI (see section 2.4) and secondly due to the need of a breathing / rebreathing system [81], which has to be used inside of the RF head coil in the scanner bore. Evaluation of $\mathrm{CMRO}_{2}$ data based on ${ }^{1} \mathrm{H}$ imaging is much easier with simple exponential fitting and basic mathematics as described in section 3.1.4.1 and in the literature $[14,77,88]$. Furthermore, no additional breathing devices are needed paving the way to easier patient handling in clinical applications. 


\section{Bibliography}

[1] A Fick. Ueber die Messung des Blutquantums in den Herzventrikeln. Sitz. Physik. Med. Ges, 2:16, 1870 .

[2] Klaus Möllenhoff, Joerg Felder, Sandro Romanzetti, A Gordji-Nejad, and N. J. Shah. Natural abundance in vivo 170 measurements at 9.4T. In ESMRMB 2013 Congress, page 326, 2013.

[3] Klaus Möllenhoff, Joerg Felder, Sandro Romanzetti, Ali Gordji-Nejad, and Nadim Jon Shah. Natural abundance in vivo 170 measurements at 9.4T. In ISMRM, 2014.

[4] K. H. M. Möllenhoff. Development of an Optimised TAPIR Sequence for T 1 and H 2 O Mapping Using Adiabatic Inversion Pulses and Parallel Imaging Techniques. PhD thesis, 2010.

[5] K. H. M. Möllenhoff, N. Jon Shah, Eberhard D. Pracht, and Tony Stöcker. Accelerated Mapping of T1 Relaxation Times Using TAPIR. In ISMRM, 2010.

[6] K. H. M. Möllenhoff, F. Keil, and N. J. Shah. Mapping of T1 using a 3D variant of TAPIR. In ESMRMB, 2011.

[7] K. H. M. Möllenhoff, N.J. J. Shah, F. Keil, M. R. Kubach, V. Gras, Z. Abbas, and A. M. O. Peusquens. Mapping of watercontent in the human brain using TAPIR. In Marie Curie Researches Symposium, 2011.

[8] K. H. M. Möllenhoff, F. Keil, and N.J. Shah. Mapping of T1 relaxation times using a 3D Variant of TAPIR. In ISMRM, 2012.

[9] K. H. M. Möllenhoff, F. Keil, M. R. Kubach, V. Gras, Z. Abbas, A. M. O. Peusquens, and N. J. Shah. Fast mapping of absolute water content in the human brain using TAPIR. In ISMRM, 2012.

[10] Zaheer Abbas, Vincent Gras, Klaus Möllenhoff, Fabian Keil, Ana-Maria Oros-Peusquens, and Nadim J Shah. Analysis of proton-density bias corrections based on T1 measurement for robust quantification of water content in the brain at 3 Tesla. Magnetic resonance in medicine : official journal of the Society of Magnetic Resonance in Medicine / Society of Magnetic Resonance in Medicine, 72(6):1735-1745, jan 2014.

[11] Zaheer Abbas, Vincent Gras, Klaus Möllenhoff, Ana-Maria Oros-Peusquens, and Nadim Joni Shah. Quantitative water content mapping at clinically relevant field strengths: A comparative study at $1.5 \mathrm{~T}$ and $3 \mathrm{~T}$. Neurolmage, 106:404-413, 2015. 
[12] D Bolar, B Rosen, A Sorensen, and E Adalsteinsson. QUantitative Imaging of eXtraction of Oxygen and TIssue Consumption (QUIXOTIC) using velocity selective spin labeling. In Proceedings 17th Scientific Meeting, International Society for Magnetic Resonance in Medicine, volume 17 , page $628,2009$.

[13] Divya Sanam Bolar. Magnetic Resonance Imaging of the Cerebral Metabolic Rate of Oxygen ( CMRO 2 ). PhD thesis, Massachusetts Institute of Technology, 2010.

[14] D S Bolar, B R Rosen, A G Sorensen, and E Adalsteinsson. QUantitative Imaging of eXtraction of oxygen and TIssue consumption (QUIXOTIC) using venular-targeted velocity-selective spin labeling. Magnetic resonance in medicine : official journal of the Society of Magnetic Resonance in Medicine / Society of Magnetic Resonance in Medicine, 66(6):1550-62, jun 2011.

[15] Hanzhang Lu and Yulin Ge. Quantitative evaluation of oxygenation in venous vessels using T2-Relaxation-Under-Spin-Tagging MRI. Magnetic resonance in medicine : official journal of the Society of Magnetic Resonance in Medicine / Society of Magnetic Resonance in Medicine, 60(2):357-63, aug 2008.

[16] Klaus Möllenhoff and Nadim Jon Shah. Improvements of quantitative oxygenation levels in venous blood (Yv) measurements based on QUIXOTIC. In ISMRM, 2015.

[17] Guofan Xu, Howard A Rowley, Gaohong Wu, David C Alsop, Ajit Shankaranarayanan, Maritza Dowling, Bradley T Christian, Terrence R Oakes, and Sterling C Johnson. Reliability and precision of pseudo-continuous arterial spin labeling perfusion MRI on 3.0 $\mathrm{T}$ and comparison with 150-water PET in elderly subjects at risk for Alzheimer's disease. NMR in Biomedicine, 23(3):286, 2010.

[18] Ana Maria Arbeláez, Yi Su, Jewell B Thomas, Amy C Hauch, Tamara Hershey, and Beau M Ances. Comparison of regional cerebral blood flow responses to hypoglycemia using pulsed arterial spin labeling and positron emission tomography. PloS one, 8(3):e60085, 2013.

[19] Ke Zhang, Hans Herzog, Jörg Mauler, Christian Filss, Thomas W Okell, Elena Rota Kops, Lutz Tellmann, Thomas Fischer, Burkhard Brocke, Walter Sturm, Heinz H Coenen, and N Jon Shah. Comparison of cerebral blood flow acquired by simultaneous [(15)O]water positron emission tomography and arterial spin labeling magnetic resonance imaging. Journal of cerebral blood flow and metabolism : official journal of the International Society of Cerebral Blood Flow and Metabolism, (April):1-8, 2014.

[20] F Bloch. Nuclear Induction. Phys. Rev., 70(7-8):460-474, oct 1946.

[21] E M Purcell, H C Torrey, and R V Pound. Resonance absorption by nuclear magnetic moments in a solid. Physical Review, 69(1-2):37-38, 1946.

[22] P C Lauterbur. Image formation by induced local interactions: examples employing nuclear magnetic resonance. Nature, 242(5394):190-191, 1973.

[23] P Mansfield. Multi-planar image formation using NMR spin echoes. Journal of Physics C: Solid State Physics, 10:L55-L58, 1977. 
[24] Raymond Damadian. Tumor detection by nuclear magnetic resonance. Science, 171(3976):1151-1153, 1971.

[25] K. H. M. Möllenhoff, Ana-Maria Oros-Peusquens, and N. Jon Shah. Introduction to the Basics of Magnetic Resonance Imaging. 1971, 2012.

[26] M.R.Thompson R W Brown R Venkatesan Haacke Mark E. Magnetic resonance imaging: physical principles and sequence design. Wiley-Liss, 1999.

[27] D G Nishimura. Principles of magnetic resonance imaging. Stanford University Stanford, 1996.

[28] A Tannús and M Garwood. Adiabatic pulses. NMR Biomed, 10(8):423-434, dec 1997.

[29] D G Norris. Adiabatic radiofrequency pulse forms in biomedical nuclear magnetic resonance. Concepts in Magnetic Resonance, 14(2):89-101, 2002.

[30] T Stöcker, K Vahedipour, D Pflugfelder, and Shah NJ. High Performance Computer MRI Simulation. Magn Reson Med, in press.

[31] www.jemris.org.

[32] Ralf Deichmann. Fast high-resolution T1 mapping of the human brain. Magn Reson Med, 54(1):20-27, jul 2005.

[33] Mark a Griswold, Peter M Jakob, Robin M Heidemann, Mathias Nittka, Vladimir Jellus, Jianmin Wang, Berthold Kiefer, and Axel Haase. Generalized autocalibrating partially parallel acquisitions (GRAPPA). Magnetic resonance in medicine : official journal of the Society of Magnetic Resonance in Medicine / Society of Magnetic Resonance in Medicine, 47(6):120210, jun 2002.

[34] P B Roemer, W A Edelstein, C E Hayes, S P Souza, and O M Mueller. The NMR phased array. Magnetic Resonance in Medicine, 16(2):192-225, 1990.

[35] Joseph W Carlson. An algorithm for NMR imaging reconstruction based on multiple RF receiver coils. Journal of Magnetic Resonance (1969), 74(2):376-380, 1987.

[36] Michael Hutchinson and Ulrich Raff. Fast MRI data acquisition using multiple detectors. Magnetic resonance in Medicine, 6(1):87-91, 1988.

[37] James R Kelton, Richard L Magin, and Steven M Wright. An algorithm for rapid image acquisition using multiple receiver coils. In Proceedings of the SMRM 8th annual meeting, Amsterdam, page 1172, 1989.

[38] J B Ra and C Y Rim. Fast imaging using subencoding data sets from multiple detectors. Magnetic resonance in medicine, 30(1):142-145, 1993.

[39] Daniel K Sodickson and Warren J Manning. Simultaneous acquisition of spatial harmonics (SMASH): fast imaging with radiofrequency coil arrays. Magnetic Resonance in Medicine, 38(4):591-603, 1997. 
[40] K P Pruessmann, M Weiger, M B Scheidegger, and P Boesiger. SENSE: sensitivity encoding for fast MRI. Magnetic Resonance in Medicine, 42(5):952-962, 1999.

[41] Peter M Jakob, Mark A Grisowld, Robert R Edelman, and Daniel K Sodickson. AUTOSMASH: a self-calibrating technique for SMASH imaging. Magnetic Resonance Materials in Physics, Biology and Medicine, 7(1):42-54, 1998.

[42] Robin M Heidemann, Mark A Griswold, Axel Haase, and Peter M Jakob. VD-AUTO-SMASH imaging. Magnetic Resonance in Medicine, 45(6):1066-1074, 2001.

[43] Mark a. Griswold, Peter M. Jakob, Robin M. Heidemann, Mathias Nittka, Vladimir Jellus, Jianmin Wang, Berthold Kiefer, and Axel Haase. Generalized Autocalibrating Partially Parallel Acquisitions (GRAPPA). Magnetic Resonance in Medicine, 47(6):1202-1210, 2002.

[44] $\mathrm{M} \mathrm{H}$ Buonocore and L Gao. Ghost artifact reduction for echo planar imaging using image phase correction. Magnetic resonance in medicine, 38(1):89-100, 1997.

[45] David A Feinberg and Koichi Oshio. Gradient-echo shifting in fast MRI techniques (ERASE imaging) for correction of field inhomogeneity errors and chemical shift. Journal of Magnetic Resonance (1969), 97(1):177-183, 1992.

[46] Da Feinberg and Koichi Oshio. Phase errors in multi-shot echo planar imaging. Magnetic Resonance in Medicine, 32(4):535-9, 1994.

[47] Robert K Murray and Joe C Davis. Harper 's Illustrated Biochemistry.

[48] G Karp. Cell and Molecular Biology: Concepts and Experiments, 7th Edition. Wiley Global Education, 2013.

[49] Patrick M S Blackett. The ejection of protons from nitrogen nuclei, photographed by the Wilson method. Proceedings of the Royal Society of London. Series A, Containing Papers of a Mathematical and Physical Character, pages 349-360, 1925.

[50] F Alder and F C Yu. On the Spin and Magnetic Moment of O 17. Physical Review, 81(6):1067, 1951.

[51] loannis P Gerothanassis. Oxygen-17 NMR spectroscopy: Basic principles and applications (part II). Progress in nuclear magnetic resonance spectroscopy, 57(1):1-110, 2010.

[52] loannis P Gerothanassis. Oxygen-17 NMR spectroscopy: basic principles and applications (part I). Progress in nuclear magnetic resonance spectroscopy, 56(1):1-110, mar 2010.

[53] G D Mateescu, G M Yvars, J C LaManna, W D Lust, and D Sudilovsky. Oxygen-17 MRS: in vivo evaluation of water uptake and residence time in the mouse brain after injection of O-17 labeled water. In Proc Inter Soc Magn Reson Med, volume 1236, 1990.

[54] Toshiyuki Arai, Kenjiro Mori, Shin-ichi Nakao, Koji Watanabe, Koichi Kito, Motoo Aoki, Hiroko Mori, Shigehiro Morikawa, and Toshiro Inubushi. In vivo oxygen-17 nuclear magnetic resonance for the estimation of cerebral blood flow and oxygen consumption. Biochemical and biophysical research communications, 179(2):954-961, 1991. 
[55] J Pekar, L Ligeti, Z Ruttner, R C Lyon, T M Sinnwell, P van Gerderen, D Fiat, C T W Moonen, and A C McLaughlin. In vivo measurement of cerebral oxygen consumption and blood flow using 170 magnetic resonance imaging. Magnetic resonance in medicine, 21(2):313-319, 1991.

[56] Daniel Fiat and Seho Kang. Determination of the rate of cerebral oxygen consumption and regional cerebral blood flow by non-invasive 170 in vivo NMR spectroscopy and magnetic resonance imaging: Part 1 . Theory and data analysis methods. Neurological research, 14(4):303-311, 1992.

[57] Xiao-Hong Zhu, Yi Zhang, Run-Xia Tian, Hao Lei, Nanyin Zhang, Xiaoliang Zhang, Hellmut Merkle, Kamil Ugurbil, and Wei Chen. Development of (17)O NMR approach for fast imaging of cerebral metabolic rate of oxygen in rat brain at high field. Proceedings of the National Academy of Sciences of the United States of America, 99(20):13194-9, oct 2002.

[58] Nanyin Zhang, Xiao-Hong Zhu, Hao Lei, Kamil Ugurbil, and Wei Chen. Simplified methods for calculating cerebral metabolic rate of oxygen based on 170 magnetic resonance spectroscopic imaging measurement during a short 1702 inhalation. Journal of cerebral blood flow and metabolism : official journal of the International Society of Cerebral Blood Flow and Metabolism, 24(8):840-8, aug 2004.

[59] Xiao-Hong Zhu, Nanyin Zhang, Yi Zhang, Xiaoliang Zhang, Kamil Ugurbil, and Wei Chen. In vivo 170 NMR approaches for brain study at high field. NMR in biomedicine, 18(2):83-103, apr 2005.

[60] Xiao-Hong Zhu and Wei Chen. In vivo oxygen-17 NMR for imaging brain oxygen metabolism at high field. Progress in Nuclear Magnetic Resonance Spectroscopy, 59(4):335-319, apr 2011.

[61] I C Atkinson and K R Thulborn. Feasibility of mapping the tissue mass corrected bioscale of cerebral metabolic rate of oxygen consumption using 17-oxygen and 23-sodium MR imaging in a human brain at $9.4\{a ́$ \}T. Neurolmage, 2010.

[62] G D Mateescu, G M Yvars, D I Pazara, N A Alldridge, J C LaManna, W D Lust, Kim McCracken, M Mattingly, and W Kuhn. Combined $17 \mathrm{O} / 1 \mathrm{H}$ magnetic resonance microscopy in plants, animals and materials: Present status and potential. International Journal of Radiation Applications and Instrumentation. Part A. Applied Radiation and Isotopes, 39(6):565, 1988.

[63] D Fiat, L Ligeti, R C Lyon, Z Ruttner, J Pekar, and A McLaughlin. Monitoring cerebral oxygen consumption in vivo 170 NMR. J Cereb Blood Flow Metab, 11(Suppl 2):781, 1991.

[64] M A Mintun, M E Raichle, W R Martin, and P Herscovitch. Brain oxygen utilization measured with $0-15$ radiotracers and positron emission tomography. Journal of nuclear medicine: official publication, Society of Nuclear Medicine, 25(2):177-187, 1984.

[65] Richard R Ernst, Geoffrey Bodenhausen, Alexander Wokaun, and Others. Principles of nuclear magnetic resonance in one and two dimensions, volume 14. Clarendon Press Oxford, 1987. 
[66] David I Hoult and R E Richards. The signal-to-noise ratio of the nuclear magnetic resonance experiment. Journal of Magnetic Resonance (1969), 24(1):71-85, 1976.

[67] X H Zhu, H Merkle, J H Kwag, K Ugurbil, and W Chen. 170 relaxation time and NMR sensitivity of cerebral water and their field dependence. Magnetic resonance in medicine : official journal of the Society of Magnetic Resonance in Medicine / Society of Magnetic Resonance in Medicine, 45(4):543-9, apr 2001.

[68] S. Meiboom. Nuclear Magnetic Resonance Study of the Proton Transfer in Water. The Journal of Chemical Physics, 34(2):375, 1961.

[69] P E Thelwall, S J Blackband, and W Chen. Field dependence of 170 T1, T2 and SNR-in vitro and in vivo studies at 4.7, 11 and 17.6 Tesla. In Proceedings of the 11th scientific meeting, International Society for Magnetic Resonance in Medicine, Toronto, volume 504, 2003.

[70] F E Boada, J S Gillen, G X Shen, S Y Chang, and K R Thulborn. Fast three dimensional sodium imaging. Magnetic resonance in medicine : official journal of the Society of Magnetic Resonance in Medicine / Society of Magnetic Resonance in Medicine, 37(5):706-15, may 1997.

[71] Hidenao Fukuyama, Masafumi Ogawa, Hiroshi Yamauchi, Shinya Yamaguchi, Jun Kimura, Yoshiaru Yonekura, and Junji Konishi. Altered cerebral energy metabolism in Alzheimer's disease: a PET study. Journal of nuclear medicine: official publication, Society of Nuclear Medicine, 35(1):1-6, 1994.

[72] Kazunari Ishii, Hajime Kitagaki, Michio Kono, and Etsuro Mori. Decreased medial temporal oxygen metabolism in Alzheimer's disease shown by PET. The Journal of Nuclear Medicine, 37(7):1159, 1996.

[73] Shigeru Yamaji, Kazunari Ishii, Masahiro Sasaki, Toru Imamura, and Others. Changes in cerebral blood flow and oxygen metabolism related to magnetic resonance imaging white matter hyperintensities in Alzheimer's disease. The Journal of Nuclear Medicine, 38(9):1471, 1997.

[74] Hidehiro lida, Terry Jones, and Shuichi Miura. Modeling approach to eliminate the need to separate arterial plasma in oxygen-15 inhalation positron emission tomography. Journal of nuclear medicine: official publication, Society of Nuclear Medicine, 34(8):1333-1340, 1993.

[75] Stefan H Hoffmann, Paul Begovatz, Armin M Nagel, Reiner Umathum, Kai Schommer, Peter Bachert, and Michael Bock. A measurement setup for direct (17) O MRI at 7 T. Magnetic resonance in medicine : official journal of the Society of Magnetic Resonance in Medicine / Society of Magnetic Resonance in Medicine, 66(4):1109-15, mar 2011.

[76] Robert Borowiak, Jens Groebner, Martin Haas, Jürgen Hennig, and Michael Bock. Direct cerebral and cardiac 170-MRI at 3 Tesla: initial results at natural abundance. Magnetic Resonance Materials in Physics, Biology and Medicine, 27(1):95-99, 2014. 
[77] P C van Zijl, S M Eleff, J a Ulatowski, J M Oja, a M Uluğ, R J Traystman, and R a Kauppinen. Quantitative assessment of blood flow, blood volume and blood oxygenation effects in functional magnetic resonance imaging. Nature medicine, 4(2):159-167, 1998.

[78] J M Oja, J S Gillen, R a Kauppinen, M Kraut, and P C van Zijl. Determination of oxygen extraction ratios by magnetic resonance imaging. Journal of cerebral blood flow and metabolism : official journal of the International Society of Cerebral Blood Flow and Metabolism, 19(12):1289-95, dec 1999.

[79] Xiao-Hong Zhu, James M Chen, Tsang-Wei Tu, Wei Chen, and Sheng-Kwei Song. Simultaneous and noninvasive imaging of cerebral oxygen metabolic rate, blood flow and oxygen extraction fraction in stroke mice. Neurolmage, 64C:437-447, sep 2012.

[80] A L Hopkins and R G Barr. Oxygen-17 compounds as potential NMR T2 contrast agents: Enrichment effects of $\mathrm{H} 217 \mathrm{O}$ on protein solutions and living tissues. Magnetic resonance in medicine, 4(4):399-403, 1987.

[81] Stefan Heinrich Hoffmann, Paul Begovatz, Armin M Nagel, Reiner Umathum, and Michael Bock. In vivo Oxygen-17 (170) MRI at 7 Tesla 1. volume 17, page 2010, 2010.

[82] Stefan H Hoffmann, Alexander Radbruch, Michael Bock, Wolfhard Semmler, and Armin M Nagel. Direct (17)O MRI with partial volume correction: first experiences in a glioblastoma patient. Magma (New York, N.Y.), c, apr 2014.

[83] Stefan Heinrich Hoffmann. Lokalisierte Quantifizierung des zerebralen Sauerstoffumsatzes (CMRO2) mit der 17O-Magnetresonanztomographie.

[84] S S Kety and C F Schmidt. The nitrous oxide method for the quantitative determination of cerebral blood flow in man; theory, procedure and normal values. The Journal of clinical investigation, 27(4):476-483, 1948.

[85] A Guyton and J Hall. The Body Fluid Compartments: Extracellular and Intracellular Fluids; Interstitial Fluid and Edema In Guyton,(2005) Textbook of Medical Physiology, 2005.

[86] K R Thulborn, J C Waterton, P M Matthews, and G K Radda. Oxygenation Dependence of the Transverse Relaxation-Time of Water Protons in Whole-Blood at High-Field. Biochimica et Biophysica Acta, 714(2):265-270, 1982.

[87] Z Luz and S Meiboom. Nuclear magnetic resonance study of the protolysis of trimethylammonium ion in aqueous solution-order of the reaction with respect to solvent. The Journal of Chemical Physics, 39(2):366-370, 1963.

[88] X Golay, M J Silvennoinen, J Zhou, C S Clingman, R a Kauppinen, J J Pekar, and P C van $\mathrm{Zij}$. Measurement of tissue oxygen extraction ratios from venous blood $\mathrm{T}(2)$ : increased precision and validation of principle. Magnetic resonance in medicine : official journal of the Society of Magnetic Resonance in Medicine / Society of Magnetic Resonance in Medicine, 46(2):282-91, aug 2001. 
[89] N J Shah, M Zaitsev, S Steinhoff, S Wiese, and K Zilles. Development of sequences for fMRI: keyhole imaging and relaxation time mapping, 2000.

[90] H Neeb, K Zilles, and N J Shah. A new method for fast quantitative mapping of absolute water content in vivo. Neuroimage, 31(3):1156-1168, 2006.

[91] H Neeb, K Zilles, and N J Shah. Fully-automated detection of cerebral water content changes: study of age-and gender-related $\mathrm{H} 2 \mathrm{O}$ patterns with quantitative MRI. Neuroimage, 29(3):910-922, 2006.

[92] J P Wansapura, S K Holland, R S Dunn, and W S Ball Jr. NMR relaxation times in the human brain at 3.0 Tesla. Journal of Magnetic Resonance Imaging, 9(4):531-538, 1999.

[93] C S Poon and R M Henkelman. Practical T2 quantitation for clinical applications. Journal of magnetic resonance imaging : JMRI, 2(5):541-53, 1992.

[94] Randall M Kroeker and R Mark Henkelman. Analysis of biological NMR relaxation data with continuous distributions of relaxation times. Journal of Magnetic Resonance (1969), 69(2):218-235, 1986.

[95] a P Crawley and R M Henkelman. Errors in T2 estimation using multislice multiple-echo imaging. Magnetic resonance in medicine : official journal of the Society of Magnetic Resonance in Medicine / Society of Magnetic Resonance in Medicine, 4(1):34-47, 1987.

[96] K Scheffler. A pictorial description of steady-states in rapid magnetic resonance imaging. Concepts in Magnetic Resonance, 11(5):291-304, 1999.

[97] Armin M Nagel, Frederik B Laun, Marc-André Weber, Christian Matthies, Wolfhard Semmler, and Lothar R Schad. Sodium MRI using a density-adapted 3D radial acquisition technique. Magnetic resonance in medicine : official journal of the Society of Magnetic Resonance in Medicine / Society of Magnetic Resonance in Medicine, 62(6):1565-73, dec 2009.

[98] Nadim Joni Shah, Heiko Neeb, Maxim Zaitsev, Sven Steinhoff, Gerald Kircheis, Katrin Amunts, Dieter Häussinger, and Karl Zilles. Quantitative T1 mapping of hepatic encephalopathy using magnetic resonance imaging. Hepatology, 38(5):1219-1226, nov 2003.

[99] Shah Et al. German Patent Number: 10028171.

[100] Shah Et al. US Patent 6803762.

[101] S Steinhoff, M Zaitsev, K Zilles, and N J Shah. Fast T(1) mapping with volume coverage. Magn Reson Med, 46(1):131-140, jul 2001.

[102] N J Shah, M Zaitsev, S Steinhoff, and K Zilles. A new method for fast multislice T(1) mapping. Neuroimage, 14(5):1175-1185, nov 2001.

[103] D C Look and D R Locker. Time saving in measurement of NMR and EPR relaxation times. Review of Scientific Instruments, 41:250, 1970.

[104] M Zaitsev, S Steinhoff, and N J Shah. Error reduction and parameter optimization of the TAPIR method for fast T1 mapping. Magn Reson Med, 49(6):1121-1132, jun 2003. 
[105] J M Gebel Jr, E C Jauch, T G Brott, J Khoury, L Sauerbeck, S Salisbury, J Spilker, T A Tomsick, J Duldner, and J P Broderick. Natural history of perihematomal edema in patients with hyperacute spontaneous intracerebral hemorrhage. Stroke, 33(11):2631, 2002.

[106] T Gerriets, E Stolz, M Walberer, C Muller, A Kluge, A Bachmann, M Fisher, M Kaps, and $\mathrm{G}$ Bachmann. Noninvasive quantification of brain edema and the space-occupying effect in rat stroke models using magnetic resonance imaging, volume 35. 2004.

[107] C Andersen. In vivo estimation of water content in cerebral white matter of brain tumour patients and normal individuals: towards a quantitative brain oedema definition. Acta neurochirurgica, 139(3):249-256, 1997.

[108] D Haussinger, J Laubenberger, S Dahl, T Ernst, S Bayer, M Langer, W Gerok, and J Hennig. Proton magnetic resonance spectroscopy studies on human brain myo-inositol in hypoosmolarity and hepatic encephalopathy. Gastroenterology-Orlando, 107(5):1475-1480, 1994.

[109] H Neeb, G Kircheis, D Haussinger, K Zilles, and N J Shah. Quantitative measurement of absolute water content in hepatic encephalopathy. Proceedings of the International Society of Magnetic Resonance in Medicine, 13:1060, 2005.

[110] P Tofts. Quantitative MRI of the brain: measuring changes caused by disease. John Wiley \& Sons Inc, 2003.

[111] Daniel Brenner. Theoretical and Experimental Analysis of Different Comtemporary Flip Angle Mapping Methods. Master's thesis, Technische Universit\{ä\}t Dortmund, 2009.

[112] V Gras, Z Abbas, and N J Shah. Experimental evaluation of RF non-uniformity correction in the mapping of the proton density. In Proceedings of the 19th Annual Meeting of ISMRM, Montreal, Canada, page 4448, 2011.

[113] V Gras, Z Abbas, A-M Oros-Peusquens, K Möllenhoff, F Keil, M Kubach, and N J Shah. Quantitative water content mapping at 1.5 \& 3 Tesla field strength. In Proceedings of the 19th Annual Meeting of ISMRM, Montreal, Canada, page 4449, 2011.

[114] D. I. Hoult. The principle of reciprocity in signal strength calculations-A mathematical guide. Concepts in Magnetic Resonance, 12(4):173-187, 2000.

[115] Tamer S. Ibrahim. Analytical approach to the MR signal. Magnetic Resonance in Medicine, 54(3):677-682, 2005.

[116] John Ashburner and Karl J. Friston. Unified segmentation. Neurolmage, 26(3):839-851, 2005.

[117] Steffen Volz, Ulrike Nöth, and Ralf Deichmann. Correction of systematic errors in quantitative proton density mapping. Magnetic Resonance in Medicine, 68(1):74-85, 2012.

[118] A-M Oros-Peusquens, F Keil, Z Abbas, V Gras, K Möllenhoff, and N J Shah. A 7 min protocol for quantitative, whole-brain, accurate water mapping at $3 \mathrm{~T}$ for neurological applications. In Proceedings of the 19th Annual Meeting of ISMRM, Melbourne, Australia, page 4270, 2012. 
[119] J Gore and R Kennan. Physical principles and physiological basis of magnetic relaxation. Stark DD, Bradley WG Jr (eds.), 1999.

[120] J B Ra, S K Hilal, and Z H Cho. A method for in vivo MR imaging of the short T2 component of sodium-23. Magnetic resonance in medicine, 3(2):296-302, 1986.

[121] D Chesler, J M Vevea, F E Boada, T Reese, C Chang, B J Barrére, A M Liu, and K R Thulborn. Rapid 3-D reconstruction from 1-D projections for metabolic MR imaging of short T2 species. In Proceedings of 11th Annual Meeting Society Magnetic Resonance in Medicine Berlin, Germany. Berkeley (CA): Society of Magnetic Medicine, page 665, 1992.

[122] Eric C Wong, Matthew Cronin, Wen-Chau Wu, Ben Inglis, Lawrence R Frank, and Thomas T Liu. Velocity-selective arterial spin labeling. Magnetic resonance in medicine : official journal of the Society of Magnetic Resonance in Medicine / Society of Magnetic Resonance in Medicine, 55(6):1334-1341, 2006.

[123] S G Kim, X Hu, and K Uğurbil. Accurate T1 determination from inversion recovery images: application to human brain at 4 Tesla. Magnetic resonance in medicine : official journal of the Society of Magnetic Resonance in Medicine / Society of Magnetic Resonance in Medicine, 31(4):445-449, 1994.

[124] Kenneth P. Whittall, Alex L. MacKay, Douglas a. Graeb, Robert a. Nugent, David K B Li, and Donald W. Paty. In vivo measurement of T2 distributions and water contents in normal human brain. Magnetic Resonance in Medicine, 37(1):34-43, 1997.

[125] H Jordan Garber, Jambur V Ananth, Lee C Chiu, Virginia J Griswold, and William H Oldendorf. Nuclear magnetic resonance study of obsessive-compulsive disorder. The American journal of psychiatry, 146(8):1001, 1989.

[126] R. M. Botnar, M. Stuber, P. G. Danias, K. V. Kissinger, and W. J. Manning. Improved Coronary Artery Definition With T2-Weighted, Free-Breathing, Three-Dimensional Coronary MRA. Circulation, 99(24):3139-3148, jun 1999.

[127] Thomas W Okell, Michael A Chappell, Michael E Kelly, and Peter Jezzard. Cerebral blood flow quantification using vessel-encoded arterial spin labeling. J Cereb Blood Flow Metab, 33(11):1716-1724, nov 2013.

[128] Ze Wang, Geoffrey K. Aguirre, Hengyi Rao, Jiongjiong Wang, María a. Fernández-Seara, Anna R. Childress, and John a. Detre. Empirical optimization of ASL data analysis using an ASL data processing toolbox: ASLtbx. Magnetic Resonance Imaging, 26(2):261-269, 2008.

[129] Michael D Abràmoff, Paulo J Magalhães, and Sunanda J Ram. Image processing with ImageJ. Biophotonics international, 11(7):36-42, 2004.

[130] Wayne Rasband (http://rsb.info.nih.gov/ij). ImageJ 1.42q.

[131] H Neeb, V Ermer, T Stocker, and N Jon Shah. Fast quantitative mapping of absolute water content with full brain coverage. Neuroimage, 42(3):1094-1109, 2008. 
[132] P J Wright, O E Mougin, J J Totman, A M Peters, M J Brookes, R Coxon, P E Morris, $\mathrm{M}$ Clemence, S T Francis, R W Bowtell, and Others. Water proton T 1 measurements in brain tissue at 7, 3, and 1.5 T using IR-EPI, IR-TSE, and MPRAGE: results and optimization. Magnetic Resonance Materials in Physics, Biology and Medicine, 21(1-2):121-130, 2008.

[133] David C Zhu and Richard D Penn. Full-brain T1 mapping through inversion recovery fast spin echo imaging with time-efficient slice ordering. Magnetic resonance in medicine, 54(3):725731, 2005.

[134] Thomas Ethofer, Irina Mader, Uwe Seeger, Gunther Helms, Michael Erb, Wolfgang Grodd, Albert Ludolph, and Uwe Klose. Comparison of longitudinal metabolite relaxation times in different regions of the human brain at 1.5 and 3 Tesla. Magnetic resonance in medicine, 50(6):1296-1301, 2003.

[135] Greg J Stanisz, Ewa E Odrobina, Joseph Pun, Michael Escaravage, Simon J Graham, Michael J Bronskill, and R Mark Henkelman. T1, T2 relaxation and magnetization transfer in tissue at 3T. Magnetic resonance in medicine : official journal of the Society of Magnetic Resonance in Medicine / Society of Magnetic Resonance in Medicine, 54(3):507-12, sep 2005.

[136] Pipe. MRI Unbound - MRI PULSE SEQUENCE DESIGN AND RECONSTRUCTION SOURCE CODE REPOSITORY, jul 2015.

[137] Ralph Beebe Blackman and John Wilder Tukey. The measurement of power spectra from the point of view of communications engineering-part I. Bell System Technical Journal, 37(1):185-282, 1958.

[138] C-h. Choi, Y Ha, P Veeraiah, K Moellenhoff, and N. J. Shah. Design and implementation of a simple multinuclear preclinical MRI system. Magnetic Resonance Materials in Physics, Biology and Medicine, 28(S1):217, 2015.

[139] K L Leenders, D Perani, a a Lammertsma, J D Heather, P Buckingham, M J Healy, J M Gibbs, R J Wise, J Hatazawa, and S Herold. Cerebral blood flow, blood volume and oxygen utilization. Normal values and effect of age. Brain : a journal of neurology, 113 ( Pt 1:27-47, 1990.

[140] P L Madsen, S G Hasselbalch, L P Hagemann, K S Olsen, J Bülow, S Holm, G Wildschiødtz, $\mathrm{O}$ B Paulson, and $\mathrm{N}$ a Lassen. Persistent resetting of the cerebral oxygen/glucose uptake ratio by brain activation: evidence obtained with the Kety-Schmidt technique. Journal of cerebral blood flow and metabolism : official journal of the International Society of Cerebral Blood Flow and Metabolism, 15(3):485-491, 1995.

[141] J C Baron, P Lebrun-Grandie, Ph Collard, C Crouzel, G Mestelan, and M G Bousser. Noninvasive measurement of blood flow, oxygen consumption, and glucose utilization in the same brain regions in man by positron emission tomography: concise communication. Journal of nuclear medicine : official publication, Society of Nuclear Medicine, 23(5):391-399, 1982. 
[142] Saeid Taheri, Charles Gasparovic, Nadim Jon Shah, and Gary A Rosenberg. Quantitative measurement of blood-brain barrier permeability in human using dynamic contrast-enhanced MRI with fast T1 mapping. Magnetic Resonance in Medicine, 65(4):1036-1042, 2011. 